\title{
Nanoscale pattern formation on ion-sputtered surfaces
}

\author{
Dissertation \\ zur Erlangung des mathematisch-naturwissenschaftlichen Doktorgrades \\ "Doctor rerum naturalium" \\ der Georg-August-Universität Göttingen
}

vorgelegt von

Taha Yasseri

aus Teheran

Göttingen, 2009 
D7

Referent: Prof. Dr. Reiner Kree

Koreferent: Prof. Dr. Alexander Karl Hartmann

Tag der mündlichen Prüfung: 21.01.2010 


\section{Abstract}

Self-organized, nano-scale structures appear on solid surfaces under ion beam irradiation with ion energies in the $\mathrm{keV}$ range. Within the last decade, surface engineering by ion beam sputtering (IBS) has become a very promising candidate for bottom-up production techniques of nano-devices. Morphologies like ripples, and regular arrays of dots, pyramids and pits as well as ultrasmooth surfaces have been obtained on a wide variety of substrates, including important semiconductor materials like Si, Ge, GaAs and InP.

In spite of many substantial improvements of experimental surface structuring by IBS, the physical mechanisms underlying the pattern formation are still poorly understood. In this work we use Kinetic Monte Carlo (KMC) simulations and continuum theory to study the effects of the following mechanisms in detail: (i) the interplay of surface erosion with different surface diffusion mechanisms (Wolf-Villain, Hamiltonian, thermally activated hopping via transition states, including barriers depending on both initial and final configuration in a hop) and the crossover from erosion-driven to diffusion driven patterns, (ii) random orientational fluctuations of ion trajectories within the beam, leading to ion beam divergence, (iii) co-deposited, steady-state, (sub)-mono-layer coverages of the substrate with a second atomic species ("surfactant sputtering") and (iv) multi-beam and rotated-beam (or rotated sample) setups. We find that all the four mechanisms under study may have a profound - and sometimes unexpected - impact on the pattern formation. Different diffusion mechanisms, which all give rise to the same leading order terms in a continuum description lead to rather different long-time behavior of patterns in KMC simulations. Orientational fluctuations change the bifurcation scenarios of pattern formation and surfactant sputtering may give rise to qualitatively new effects like mesoscopic or even macroscopic patterns on top of nano-scale patterns, and the ordering of the surfactant on top of the structured surface. This ordering leads to a feedback mechanism due to the modulation in sputtering yield caused by the surfactant. On the other hand, many of the promising proposals concerning the usage of multi-beam and rotated beam setups could not be confirmed (in accordance with recent experiments), but we can outline some physical reasons for this failure, which could guide an improved usage of these techniques. 


\section{Dedication}

To Maman and Baba. 


\section{Acknowledgment}

First and foremost I would like to thank Prof. Dr. Reiner Kree, who was my thesis adviser. His sharp advice opens a wide window to all aspects of the topic, while he always kindly let me follow my own ideas as well. I enjoyed cooperating with him a lot and (hopefully) learned much from him.

I would also like to thank Prof. Dr. Alexander K. Hartmann, who supervised the first 6 month of my research in Göttingen and kept supporting me even after he moved to Oldenburg, and Dr. Emanuel O. Yewande, who kindly provided the KMC codes he had written during his $\mathrm{PhD}$, and let my research reach the productivity level very soon.

As I have been working on this thesis, have been fortunate to have nice discussions with Prof. Dr. Hans Hofsäss, Dr. Kun Zhang and Prof. Dr. Rodolfo Cuerno.

It has been a great pleasure to work in the Institute for Theoretical Physics, and I would like to thank all its past and present members, specially, Prof. Dr. Kurt Schönhammer (head of the institute), Prof. Dr. Annette Zippelius, Prof. Dr. Marcus Müller and Dr. Jürgen Holm, and then all my colleagues and officemates; Dr. Bernd Burghardt, Andrea Fiege, Till Kranz, Alexander Mann, Kristian Marx, Dr. Stefan Wolfsheimer and Martin Zumsande. Here, my deepest thanks go to Oliver Melchert.

I appreciate all the efforts of the secretary team of the institute, Frauen Glormann, Lütge-Hampe and Schubert, and the SFB secretary Frau Hühne, to facilitate the administrative processes. I'm also deeply thankful to the faculty secretary Frau Afshar.

I must confess that my residence in Göttingen would not be so pleasant without the support of my dearest Iranian friends (spread all around the world) . Therefore I would like to thank Talayeh Aledavood (Munich), Homa Ghalei, 
Sara Hadji Moradlou (Saarbrücken), Dr. Nima Hamedani Radja (Leiden), Majid Hojjat (Munich), Sona Nazari (Tehran), Mahmoudreza Saadat (Stanford), Shahriar Shadkhoo (Los Angeles). I especially appreciate all scientific and non-scientific supports from Armita Nourmohammad (Cologne).

Sohail Khoshnevis, Amgad Squires and Lishia Teh, each of them has kindly corrected parts of the manuscript of this thesis. I am very grateful for that.

This work was funded by the German research foundation, the Deutsche Forchungsgemeinscaft (DFG), within the Sonderforchungsbereich (SFB) 602: Complex Structures in Condensed Matter from Atomic to Mesoscopic Scales.

The simulations were performed at the workstation cluster of the "Institute for Theoretical Physics", university Göttingen. 


\section{List of abbreviations}

$\AA$ : Ångstrom

AES: Auger electron spectroscopy

AFM: atomic force microscopy

BCA: binary collision approximation

BH: Bradley-Harper

DIBS: dual-ion-beam sputtering

DT: Das Sarma-Tamborenea

ES: Ehrlich-Schwoebel

$\mathrm{eV}$ : electron volt

EW: Erdwards-Wilkinson

HKGK: Hartmann-Kree-Geyer-Kölbel

IBS: ion-beam sputtering

KMC: kinetic Monte Carlo

KS: Kuramoto-Sivashinsky

LC: larger curvature

LD: Lai-Das Sarma

MBE: molecular beam epitaxy

MCB: Makeev-Cuerno-Barabási

MD: molecular dynamics

RIBS: rotational ion-beam sputtering

SEM: scanning electron microscopy

SIBS: sequential ion-beam sputtering

SIMS: secondary ion mass spectrometry

SOS: solid-on-solid 
viii

STM: scanning tunneling microscopy

WV: Wolf-Villain 


\section{Contents}

1 Introduction 1

2 Ion-beam sputtering experiments 5

2.1 Patterns on amorphous substrates . . . . . . . . . . . . 5

2.1 .1 Ripples . . . . . . . . . . . . . . . . 6

2.1 .2 Dots . . . . . . . . . . . . . . . . . 8

2.1 .3 Holes . . . . . . . . . . . . . . . . . . . . . . . . . . . 11

2.1.4 Smooth surfaces . . . . . . . . . . . . . . . . 11

2.2 Patterns on crystalline substrates . . . . . . . . . . . . . 14

2.3 Advanced patterning methods . . . . . . . . . . . . . . . 14

2.3 .1 Surfactant sputtering . . . . . . . . . . . . . . 16

2.3.2 Compound beams . . . . . . . . . . . . . . . . 16

3 Methods 19

3.1 Binary collision approximation . . . . . . . . . . . . . 20

3.1 .1 Cascade shape . . . . . . . . . . . . . . . . . 21

3.1.2 Defect generation . . . . . . . . . . . . . . . 22

3.1 .3 Down-hill current . . . . . . . . . . . . . . . . 22

3.2 Kinetic theory . . . . . . . . . . . . . . . . . . . . . . 25

3.3 Kinetic Monte Carld . . . . . . . . . . . . . . . 25

$3.3 .1 \quad$ Erosion $\ldots \ldots \ldots \ldots \ldots \ldots$

3.3 .2 Diffusion . . . . . . . . . . . . . . . . 26

3.4 Continuum theorv . . . . . . . . . . . . . . . . . . . . 28

$3.4 .1 \quad$ Bradlev-Harper model . . . . . . . . . . . . . . . . . 28

3.4.2 Cuerno-Barabási non-linear model . . . . . . . . . . . . . 30

3.4.3 Makeev, Cuerno and Barabási model . . . . . . . . . . . 31 
3.4.4 Non-local linear stability analysis . . . . . . . . . . . 32

3.5 Data analvsis ... . . . . . . . . . . . . . . . 33

3.5.1 Scaling analvsis . . . . . . . . . . . . . . 33

3.5.2 Power spectral density . . . . . . . . . . . . . . . . . 34

4 Erosion and diffusion-driven patterns 37

4.1 Erosion . . . . . . . . . . . . . . . . . . . . 38

4.1.1 Dependence of patterns on ion parameters . . . . . . . 38

4.1 .2 BCA model based erosion . . . . . . . . . . . . . 41

4.2 Diffusion . . . . . . . . . . . . . . . . . . . . . 41

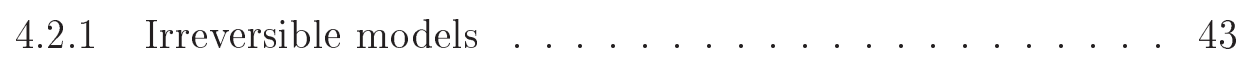

4.2 .2 Hamiltonian models . . . . . . . . . . . . . . . 45

4.2.3 Thermally activated models . . . . . . . . . . . . . 47

4.2.4 Ehrlich-Schwoebel effects, pattern formation by diffusion 49

4.2.5 Diffusion in compound svstems, clustering . . . . . . . . 51

4.3 Erosion-Diffusion interplav . . . . . . . . . . . . . 52

4.3.1 Dependence of patterns on diffusion in the erosive regime 55

4.3.2 Crossover from erosive regime to diffusive regime . . . . 60

5 Surfactant Sputtering $\quad 65$

5.1 Implementation . . . . . . . . . . . . . . . . 66

5.1 .1 Continuum theory . . . . . . . . . . . . 67

5.1 .2 KMC model . . . . . . . . . . . . . . . . . . 69

5.2 Mesoscopic height gradient . . . . . . . . . . . . . . . 70

5.3 Morphology modification . . . . . . . . . . . . . . . . 72

5.3.1 Ultra-smooth surfaces . . . . . . . . . . . . . 72

5.3 .2 Arravs of nano-clusters . . . . . . . . . . . . 73

5.3 .3 ES induced patterns . . . . . . . . . . . . . 77

$\begin{array}{lll}6 & \text { Beam-noise induced effects } & 79\end{array}$

6.1 Homogeneous sub-beams . . . . . . . . . . . . . . . 81

6.2 Temporally fluctuating homogeneous beams . . . . . . . . . 82

6.3 Spatio-temporally fluctuating beams . . . . . . . . . . . 83

6.3.1 Normal incidence angle . . . . . . . . . . . . . 85

6.3.2 Grazing incidence angle . . . . . . . . . . . . 85 
6.3.3 High diffusion rate regime . . . . . . . . . . . . . 87

7 Compound sputtering setups $\quad 89$

7.1 Simulation setup . . . . . . . . . . . . . . . . 9 90

7.2 Opposed ion-beams . . . . . . . . . . . . . . . . . . . . 92

7.3 Crossed ion-beams . . . . . . . . . . . . . . . . . . . 92

7.4 Sputtering of rippled surfaces _... . . . . . . . . . . . . 98

7.5 Sputtering of continuously rotating sample . . . . . . . . . . . 100

8 Conclusion 109

$\begin{array}{ll}\text { A Directional noise } & 113\end{array}$ 


\section{List of Figures}

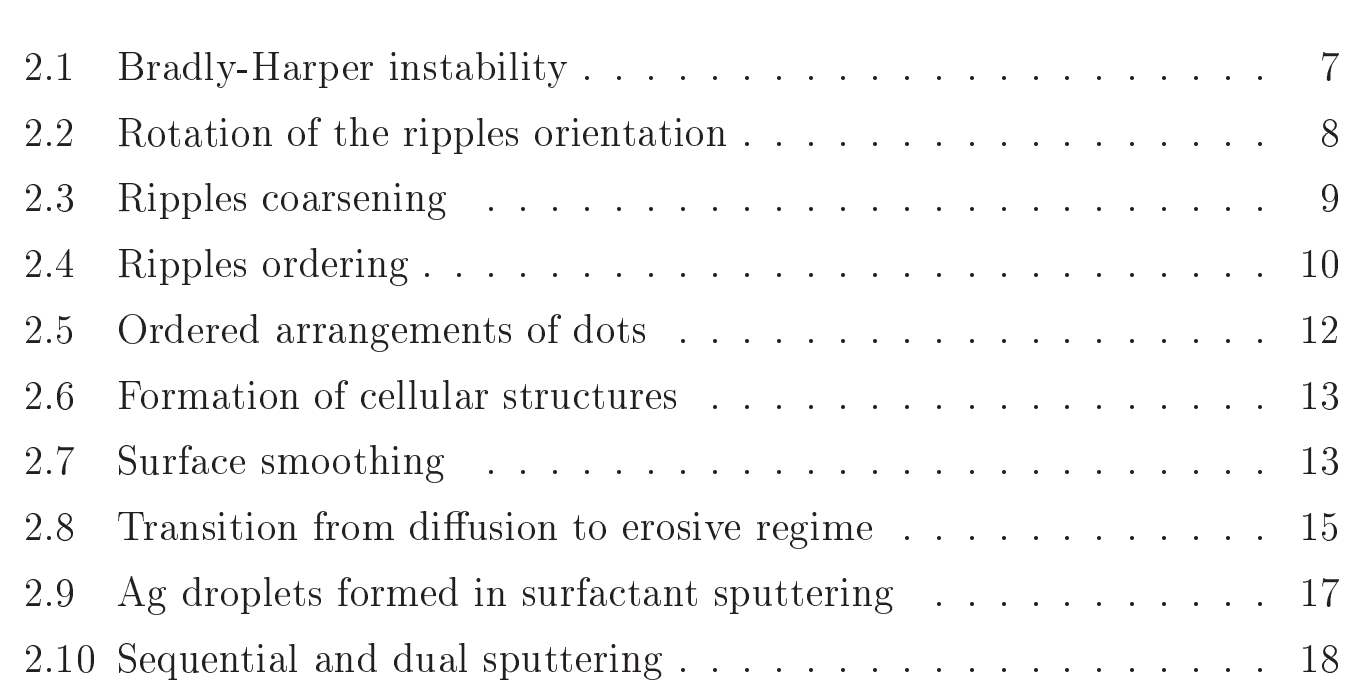

3.1 Collision cascade and its spatial energy distribution . . . . . . . 21

3.2 Energy distribution of cascades atoms . . . . . . . . . . . . 23

3.3 Down-hill current induced by collision cascades . . . . . . . . . 24

3.4 Effective surface tensions . . . . . . . . . . . . . . . . 30

3.5 Power spectral density analysis . . . . . . . . . . . 35

4.1 Kinetic phase diagram of patterns . . . . . . . . . . . . . 39

4.2 Different topographies merging from different erosion parameters 40

4.3 Pattern formation dependence on energy distribution . . . . . . 42

4.4 Ehrlich-Schwoebel barrier . . . . . . . . . . . . . . 50

4.5 Ehrlich-Schwoebel induced patterns in MBE . . . . . . . . . . . 51

4.6 Clustering on flat template . . . . . . . . . . . . . . . . 53

4.7 Clustering on sinusoidal template . . . . . . . . . . . . . 54

4.8 Ripples evolution in Hamiltonian model . . . . . . . . . . . . 56

4.9 Ripples evolution in Arrhenius (net-bond-breaking) model . . . 57

4.10 Long time morphologies emerge from different diffusion models . 58 


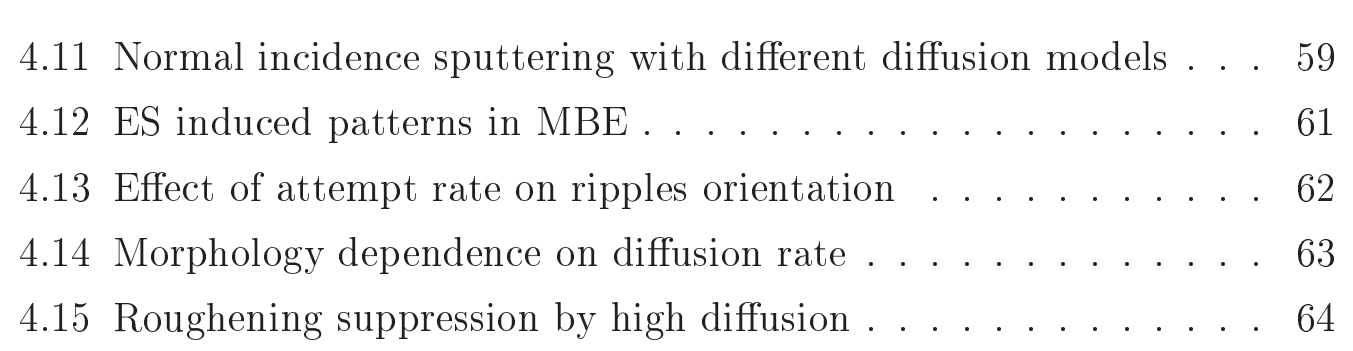

5.1 Surfactant sputtering experimental setup . . . . . . . . . . . 66

5.2 Morphologies modified by identical surfactants . . . . . . . . . 70

5.3 Patterns wavelength vs. the coverage of identical surfactants . . 71

5.4 Roughness of profiles evolved in surfactant sputtering . . . . . . 71

5.5 Mesoscopic height gradient bv surfactant sputtering . . . . . . . 72

5.6 Surface smoothing by surfactant sputtering . . . . . . . . . . . 74

5.7 Surface smoothing bv surfactant sputtering: varving coverage . . 75

5.8 Nano-wires produced by surfactant sputtering . . . . . . . . . 76

5.9 Surfactant densitv in different heights . . . . . . . . . . . 77

5.10 Ehrlich-Schwoebel induced patterns in surfactant sputtering . . 78

6.1 Different tvpes of beam-noise . . . . . . . . . . . . 80

6.2 Simulated beam profile . . . . . . . . . . . . . . . 81

6.3 Growth rate as a function of beam-divergence . . . . . . . . . . 82

6.4 Renormalaized surface tensions due to the beam-noise . . . . . . 84

6.5 Noise effects on surface evolution rate . . . . . . . . . . . . 85

6.6 Noise induced effects in normal-incidence sputtering . . . . . . . 86

6.7 Length scale selection by beam-noise in normal incidence . . . . 86

6.8 Beam-noise effects in grazing incidence angle . . . . . . . . . . . 87

6.9 Beam-noise effects in high rate diffusion regime . . . . . . . . 88

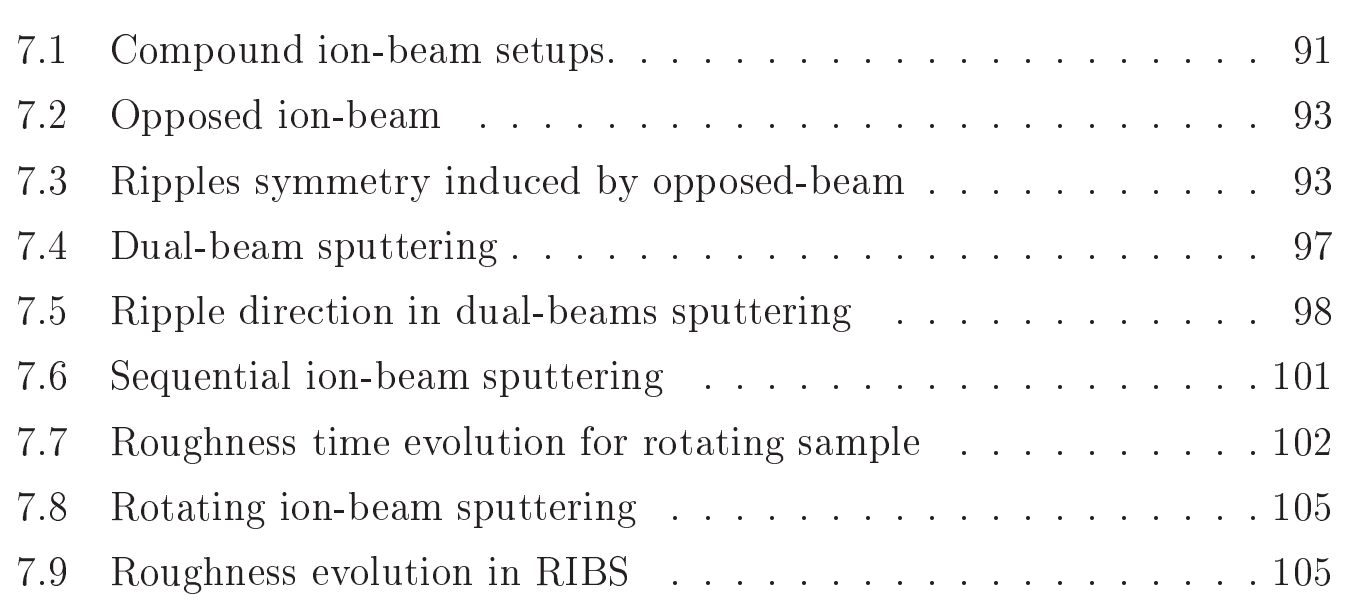


7.10 Rotation frequencv dependence of roughness in RIBS . . . . . . 106 7.11 Bradlev-Cirlin theorv of RIBS . . . . . . . . . . . . . 106 7.12 Integrated vield vs. rotation frequency in RIBS . . . . . . . . 107 


\section{List of Tables}

4.1 Scaling exponents of irreversible discrete diffusion models . . . . 46

4.2 Hopping attempt frequency at different temperatures . . . . . . 49

7.1 Beam parameters for the DIBS setup . . . . . . . . . . . 96 


\section{Chapter 1}

\section{Introduction}

Richard P. Feynman attracted the attention of scientists and engineers to the enormous capability of the nano-scale world for the first time. He gave an insight to the great possibilities, provided by instruments, which are designed and fabricated in the size of some nanometers in his talk at the annual meeting of the American Physics society 1 During the last 50 years, numerous different techniques and methods to manipulate materials on nano-scales are presented and nowadays a multitude of nano-devices are produced and available in the markets (Maynard et al. 2006).

Wide ranges of applications are proposed by nano-structures fabricated on solid surfaces. Among all the available techniques for the fabrication of such structures, e.g. chemical lithography and atomic force microscopy (AFM) techniques, bottom-up self-organized patterning methods are of particular interest, because they bear the potential of cheap, large-scale production. Ion-beam sputtering (IBS) was introduced by Navez et al. (1962) as a simple method for preparing wave-like patterns (ripples) of sub-micrometer length scales on the surface of solids. In this method, surface bombardment by a beam of keV ions at normal or oblique incidence drives the system towards self-organized formation of nano-patterns. Later on, many experimental developments have been carried out to improve the quality of the patterns i.e. ripple alignment and regularity. Meanwhile, by sputtering different kinds of solids under different conditions, new types of patterns have been discovered. The production of

\footnotetext{
${ }^{1}$ December 29th 1959, California Institute of Technology (Caltech), "There's Plenty of Room at the Bottom".
} 
regular arrays of nanometer-sized columns (dots) is one prominent example of this kind (Facsko et al. 1999). Recent reviews summarize the state of the art of surface engineering by IBS (Frost et al. 2008).

Although great improvement achieved in experiments to produce various highly ordered patterns on a wide range of different materials, a comprehensive understanding of the physical mechanisms underlying this self-organized pattern formation is not yet available. The simplest quantitative theory of IBS-induced pattern formation has been put forward in a seminal paper by Bradley and Harper (1988). There, it is pointed out that IBS implies a generic curvature instability, which roughens the surface. The combined action of this instability and surface diffusion leads to the appearance of ripples. This continuum theory has been extended in many different ways, but recent experiments indicate that it does not contain all the physical mechanisms, which determine ripple patterns on solid surfaces (Chan and Chason 2007).

In this work we aim to address the IBS problem by analytical and computational methods. We develop and use a Kinetic Monte Carlo (KMC) model for ion-beam erosion inspired by the kinetic theory of Sigmund (1969). We simulate a simple cubic lattice, which undergoes bombardment of ions with variable energy and incidence angle. We try to give new insights into physical mechanisms of IBS-driven patterns as well as examine new possibilities to improve and advance IBS experiments to achieve more precise control of the pattern formation process.

In the next chapter we give a review of recently developed experimental methods in addition to classic IBS techniques of ion-beam surface etching.

In Chapter 3 we introduce the analytical and numerical methods we use to study IBS, especially our KMC model and its basic assumptions and the continuum description of IBS.

It is the common belief that patterns under IBS form due to a competition between surface roughening (by erosion) and smoothing (by surface diffusion). In the simple continuum description of Bradley and Harper, these mechanisms enter in universal forms and are quantified by three parameters, two for erosion and one for surface diffusion. But does this exhaust the interplay of different surface diffusion mechanisms with ion-beam erosion? This question will be addressed in Chapter 4 . There, we present our results obtained by implement- 
ing different erosion and diffusion models and show how by tuning the rate of erosion and diffusion events different types of instability leading to various kind of patterns can be induced.

In Chapter 5 we present results on IBS accompanied by the co-deposition of a second atom species onto the surface. Controlled co-deposition of (sub-) mono-layer coverages by co-sputtering of a nearby target has been introduced by Hofsäss and Zhang (2008), who called it "surfactant sputtering". Meanwhile there are many indications that highly ordered regular patterns, which have been produced in experiments, have in fact involved co-deposition, which went unnoticed. We show some effects induced by co-deposition of metallic atoms on the surface of a substrate like Silicon. We demonstrate the possibility of preparing nano-clusters by this method and control them in a pattern of ripples.

In Chapter 6 we study the effects induced by including exterior noise, originated from fluctuations of the directions of ion trajectories within the beam. Our extended continuum model predicts new transitions for pattern morphologies which differ from the standard scenario of Bradley and Harper. Our results obtained by KMC simulations are about the generic effects due to the ion-beam noise in different situations, e.g. normal and grazing incidence angle or in high temperature regimes.

There are a number of proposals, mostly based on qualitative reasoning, how to improve or modify pattern formation due to IBS by using multi-ion-beam setups, sequential sputtering of the sample from different directions, or sample rotation. Chapter 7 contains a detailed simulation study of these proposals. We compare our results with recent experimental findings by Joe et al. (2008).

Finally, in Chapter 8 we give a conclusion and discussion on all presented results and some outlooks for future work. 


\section{Chapter 2}

\section{Ion-beam sputtering experiments}

Depending on the sputtering parameters e.g. flux, energy, type and incidence angle of the ions, as well as substrate properties e.g. type and substrate temperature, a wide range of different patterns might emerge via IBS. Secondary features, like beam-profile and rotation of sample may also change the qualitative and quantitative characteristics of the patterns. Recent advanced methods in IBS experiments that might produce more complex textures on the surface of materials are based on setups composed of doubled- or multi-beams, simultaneously co-sputtering of metallic and non-metallic substrates (surfactant sputtering), and sputtering of pre-structured templates. In the following sections of this chapter, we briefly review the experimental achievements of IBS. For more extended reviews see Valbusa et al. (2002), Frost et al. (2008) and Muñoz-García et al. (2009).

\subsection{Patterns on amorphous substrates}

Most of the IBS experiments are performed on amorphous substrates e.g. glass, or substrates which are amorphized under bombardment of keV ions e.g. Si, $\mathrm{SiO}_{2}, \mathrm{GaSb}$, InP, etc. Ripples and -more recently- dots are the main types of patterns which emerge on these types of substrates. Moreover, formation of holes and pits, the appearance of ultra-smooth surfaces and non-structured rough surfaces are also reported as outcomes of some IBS experiments. In the following, the mentioned types of structures are discussed in more detail. 


\subsubsection{Ripples}

A first experiment by Navez et al. (1962) was followed by a huge number of experiments, in which wave-like ripple structures were observed. The periodicity of ripples varies from tens to hundreds of nanometers and their length can exceed several micrometers. Some universal properties are observed in experiments with very different ion-beam and substrate parameters. Ripple orientation with respect to ion-beam direction and the dependence of this orientation on the incidence angle of the ion-beam with respect to the surface normal $\theta$, are important universal features, which have been explained by theory of Bradley and Harper (BH). In BH theory, different erosion rates at different points on the surface in relation to the local curvatures is the main destabilizing factor which is shown to be sufficient to explain the formation of ripples and their orientation. A schematic drawing of $\mathrm{BH}$ theory is presented in Fig. 2.1. The full description of the theory is provided in section 3.4.1. Other universal features, like the direction of ripple on crystalline substrates, propagation and the coarsening of ripple patterns are not fully understood within $\mathrm{BH}$ theory, but extensions of this theory lead to partial understanding of many features of the pattern formation (see section 3.4).

The quality of ripples, i.e. their regularity and alignment, improved tremendously within the last decade (compare the structures depicted in Fig. 2.2 and Fig. 2.4). However, so far, no comprehensive explanation on the conditions and physical mechanisms, which lead to the formation of such "ultra-regular" patterns exists. Two main ideas that may explain this experimental success are (i) fine tuning of ion-beam profile and (ii) manipulations in the process of pattern formation by co-deposited metallic atoms. Both ideas are discussed extensively in this thesis in chapters 5 and 6 respectively.

\section{Orientation}

The orientation of ripples is typically confined to be either parallel or perpendicular to the projection of the ion-beam direction onto the surface. For small values of incidence angle $\theta$, orientation is perpendicular to the ion-beam. By increasing $\theta$ towards grazing incidence, at some critical value $\theta_{c}$, ripples rotate by $90^{\circ}$ and align in direction parallel to the ion-beam. One exam- 


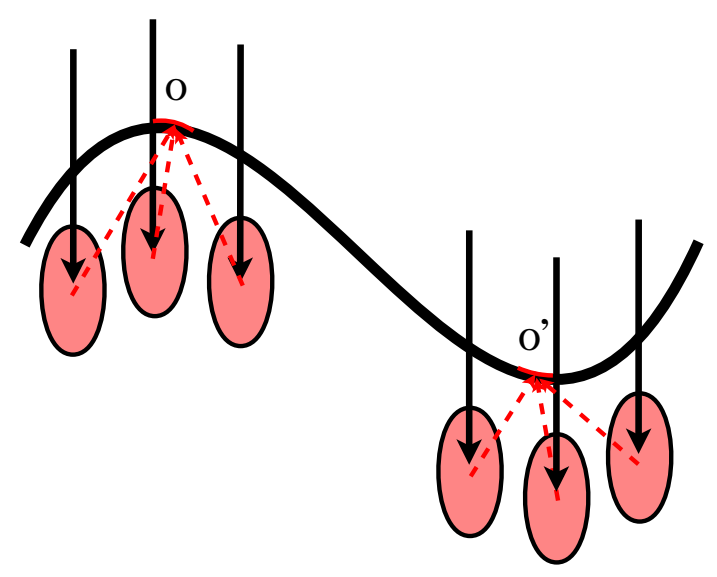

Figure 2.1: Ions hit the surface with normal incidence angle, impinge into the surface, stop at some distance under the surface and deposit their kinetic energy. Since the amount of the deposited energy reaching the lateral points with positive or negative local curvatures $(\mathrm{O}$ or O') is different, assuming the erosion rate at surface points is proportional to the reaching energy, a faster erosion rate is expected in valleys (point o'). This leads to amplification of the initial surface roughness (Bradley and Harper 1988).

ple of the change in orientation in the experiments on graphite samples by Habenicht et al. (1999) is shown in Fig. 2.2.

\section{Propagation}

In some experiments (Habenicht et al. 2002; Alkemade 2006) by simultaneous real time monitoring of pattern evolution, it is observed that ripples propagate along the direction of the ion-beam. Initial movements with velocity of 0.33 $\mathrm{nm} \mathrm{s}{ }^{-1}$ are followed by deceleration and a dispersion in velocity for different wavelength. At longer times, faster movements for ripples with shorter wavelength was reported. Ripple propagation is also predicted by $\mathrm{BH}$ theory, but the predicted direction of motion is apposite to the observed direction.

\section{Coarsening}

The coarsening of ripples, i.e. increase in lateral size and spacing of ripples, has been observed in a large number of experiments. A growth of wavelength, following a power law in the form of $\lambda \sim t^{0.5}$ is reported by Habenicht et al. (2002), whereas an exponential growth is found by Brown and Erlebacher (200 

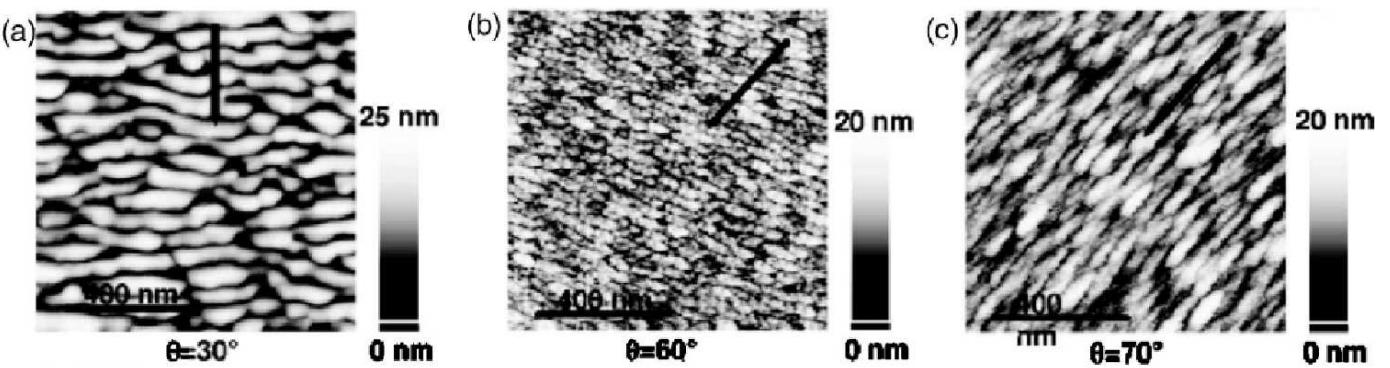

Figure 2.2: Rotation of the ripples orientation by increasing the incidence angle. Scanning tunneling microscope (STM) micro-graphs (lateral size $1 \mu \mathrm{m}$ ) of $5 \mathrm{keV} \mathrm{Xe}{ }^{+}$eroded HOPG surfaces. Fluence $=3 \times 10^{17}$ ions $/ \mathrm{cm}^{2}$; incident angle $\theta$ (a) $30^{\circ}$, (b) $60^{\circ}$ and (c) $70^{\circ}$. Arrows indicate the ion-beam orientation. Adapted from Habenicht et al. (1999).

In Fig. 2.3 an example of coarsening of ripples formed on fused silica is depicted (Flamm et al. 2001). The coarsening can only be explained by considering the non-linearities which are absent in the BH model.

\section{Ordering}

In some experiments, ripples show a tendency to become more aligned and ordered. In many experiments the number of defects (misalignment or crossing between ripples) decreases with time. For example Ziberi et al. (2005) have seen ordering and decrease of defects in sputtering on $\mathrm{Si}$ by $\mathrm{Kr}^{+}$ions (see Fig. (2.4). The order of ripples can be estimated by the counting the number of peaks in the Fourier spectrum of the surface profile.

\subsubsection{Dots}

Formation of nano-dots is another phenomenon reported in several experiments. As the anisotropy induced by the direction of the ion-beam is eliminated, either by rotating the sample (Frost et al. 2000) or by normal-incidence sputtering (Facsko et al. 1999), formation of dots is observed. However, dot formation under oblique incidence irradiation and also without sample rotation has also been reported by Ziberi et al. (2006) on Ge. The dots are highly ordered in size and have short-range ordering in placement (see Fig. 2.5). In the mentioned works, the dot patterns show hexagonal symmetry in lateral order- 

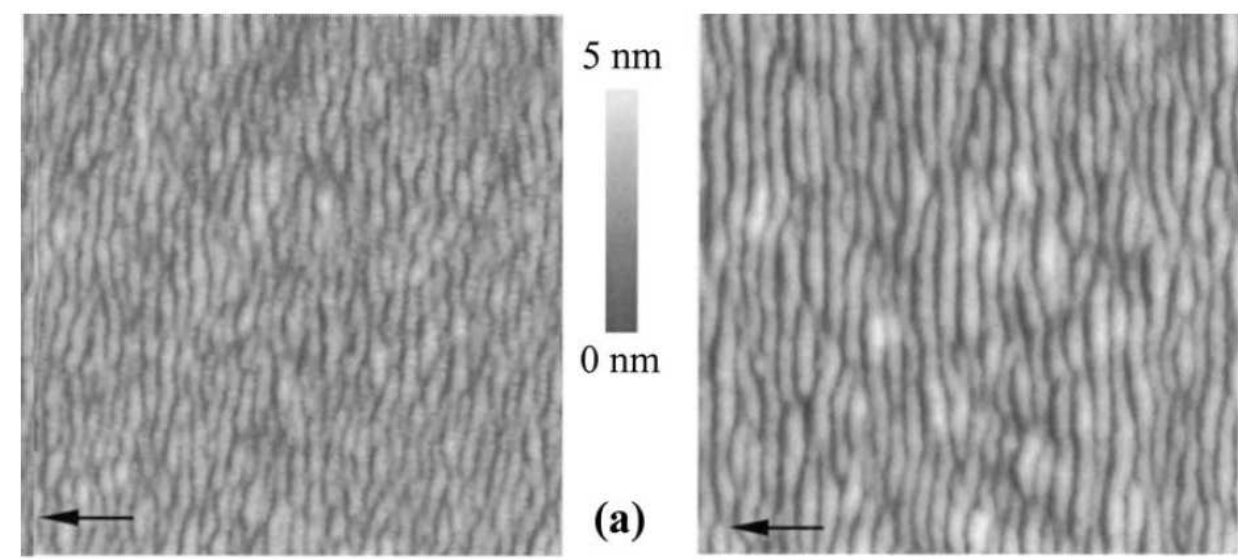

$6 \mathrm{~nm}$
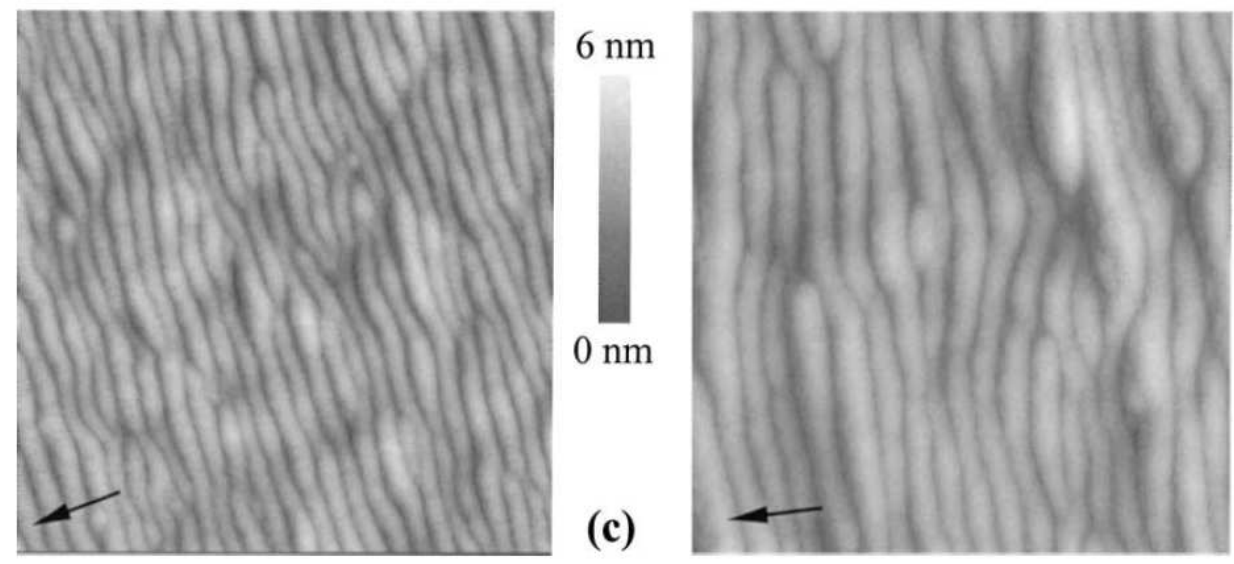

$16 \mathrm{~nm}$

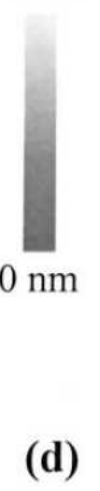

Figure 2.3: AFM image sequence, showing the evolution of fused silica surface topography with increasing sputter time $t$ at 2, 6, 10, and $60 \mathrm{~min}$, respectively. The ion-beam parameters: $800 \mathrm{eV} \mathrm{Ar}$, flux $=400 \mu \mathrm{A} / \mathrm{cm}^{2}$ and $\theta=60^{\circ}$. The lateral size of the images is $1 \mu \mathrm{m}$. The wavelength of ripples increases with time as $\lambda \sim t^{\gamma}$ with $\gamma=0.15 \pm 0.01$. Adapted from Flamm et al. (2001). 

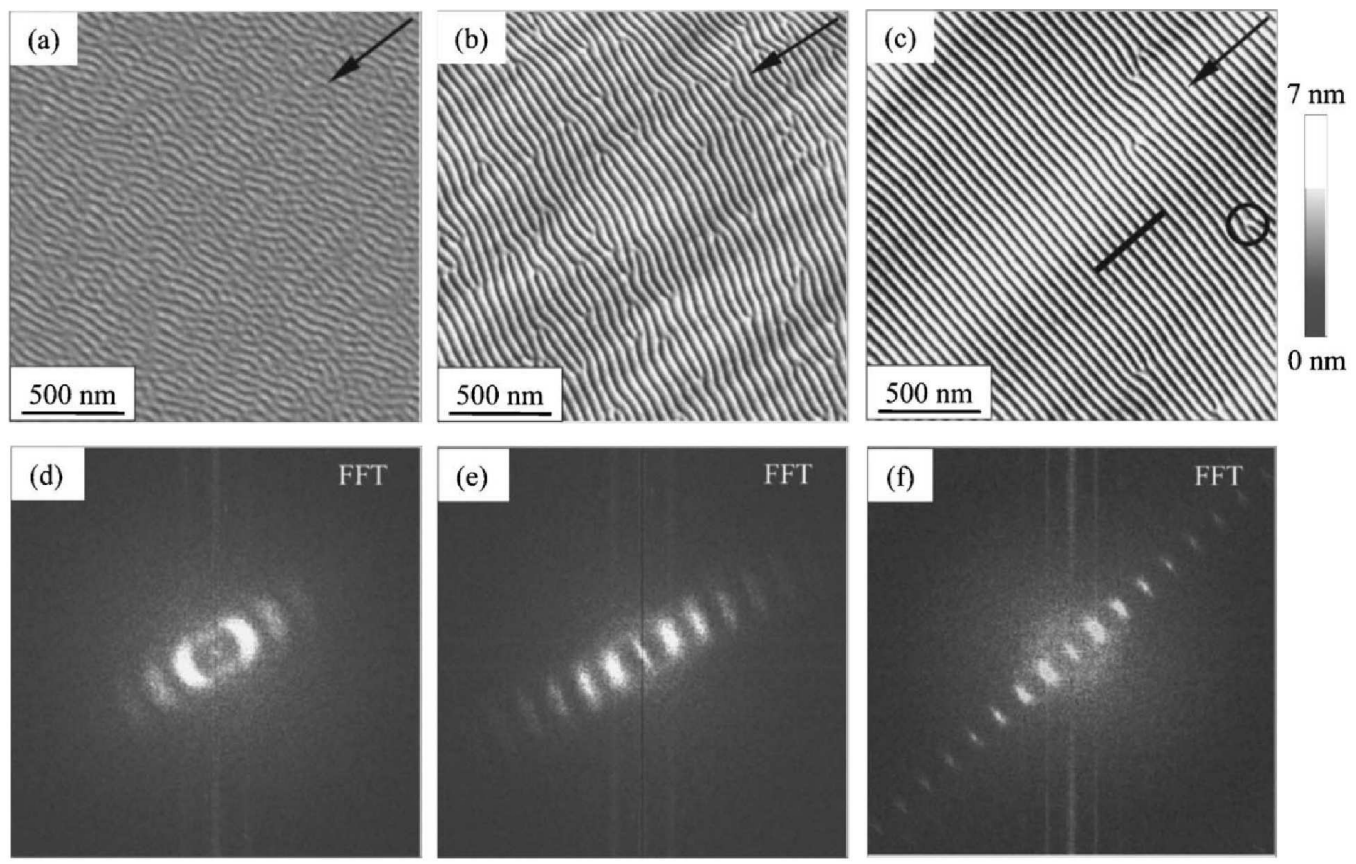

Figure 2.4: Self-organized Si ripple patterns produced by $1200 \mathrm{eV} \mathrm{Kr}{ }^{+}$ion-beam erosion, $\theta=15^{\circ}$, for different ion fluences: (a) $3.36 \times 10^{17}$ ions $/ \mathrm{cm}^{2}$, (b) $2.24 \times 10^{18}$ ions $/ \mathrm{cm}^{2}$ and (c) $1.34 \times 10^{19}$ ions $/ \mathrm{cm}^{2}$ (a)-(c) $2 \mu \mathrm{m} \times 2 \mu \mathrm{m}$ AFM images (the arrows give the ion-beam direction). (d)-(f) Corresponding Fourier spectrum (image range $\pm 127.5 \mu \mathrm{m}^{-1}$ ). The circle in (c) shows a defect in the AFM image. The number of defects decreases with time. Moreover, the angular width of the Fourier peak decreases with erosion time meaning the homogeneity and ordering (alignment) of ripples improves. Adapted from Ziberi et al. (2005). 
ing, whereas some authors reported the formation of dot patterns with square symmetry (Frost et al. 2004; Ziberi et al. 2009). The coarsening behavior of the ripples is also observed for dots, although in the case of dot patterns, an early growth in wavelength saturates in longer times (Gago et al. 2001). The formation of ordered dot patterns is not explained by BH theory.

\subsubsection{Holes}

The so-called "cellular structures" or "holes" can be produced in experiments with amorphous substrates. Fig. 2.6 shows data from Frost et al. (2004) in experiments on Si surface irradiated by $500 \mathrm{eV} \mathrm{Ar}^{+}$with sample rotation. The appearance of cellular structures in the experiments with sample rotation can be very sensitive to the rotation frequency of the sample. We discuss this in section 7.5 .

\subsubsection{Smooth surfaces}

Besides its applications for texturing the surfaces, IBS techniques can also be used for surface smoothing at microscopic length scales and for the preparation of ultra-smooth surfaces. Conventionally, continuous rotation of the sample or the ion-beam has been proposed to suppress ripple formation (Zalar 1985, Zalar 1986) in secondary ion mass spectrometry (SIMS) and Auger electron spectroscopy (AES), where ripple formation would reduce the depth profiling resolution. There are many reports on experiments with or without sample rotation, in which the initial roughness of a the surface is reduced as the sputtering process goes on (for two recent works see (Headrick and Zhou 2009) and (Frost et al. 2009)) ). In Fig. 2.7 (adapted from the latter work ) initial topography of an InSb sample is compared to snapshots after 10 and 120 min sputtering by $500 \mathrm{eV} \mathrm{N}^{+}$ions at normal incidence angle with ion current density $200 \mu \mathrm{A} / \mathrm{cm}^{2}$.

There is a lack of theory to explain the smoothing of surfaces by ionbeam techniques. In low flux and high temperature experiments, similarities to epitaxial layer-by-layer growth is claimed to exist in IBS experiments (Chan and Chason 2007). In medium and high flux experiment, a down-hill current induced by the collision cascades initiated by the impinging ions can 


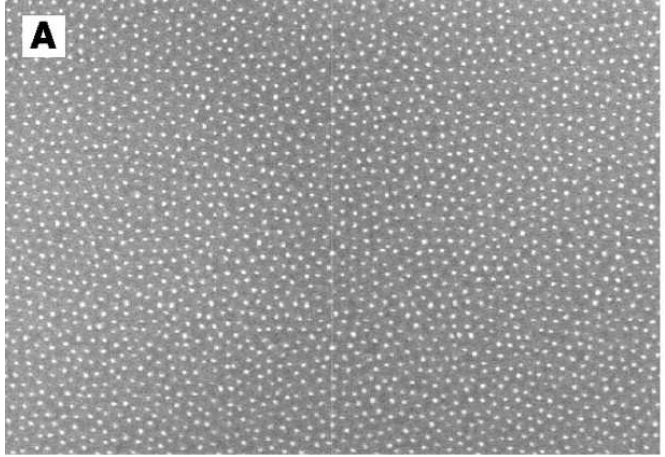

$500 \mathrm{~nm}$

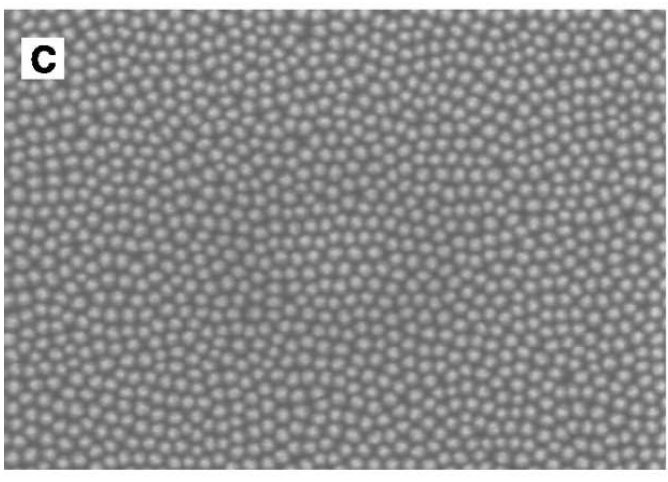

$500 \mathrm{~nm}$

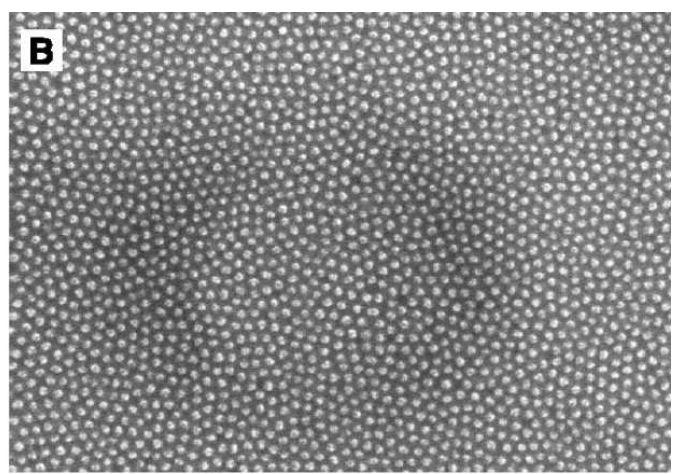

$500 \mathrm{~nm}$

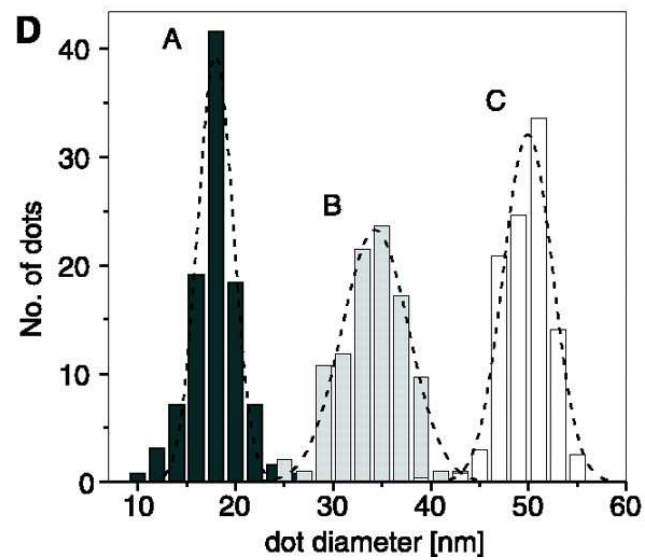

Figure 2.5: Scanning electron microscope (SEM) images of highly ordered cones on a (100) GaSb surface show the temporal evolution of dot formation during ion sputtering. The nano-scale patterns are depicted for different ion fluences (exposure times) of (A) 4 $\times 10^{17} \mathrm{ion} / \mathrm{cm}^{2}(40 \mathrm{~s}),(\mathrm{B}) 2 \times 10^{18}$ ions $/ \mathrm{cm}^{2}(200 \mathrm{~s})$, and (C) $4 \times 10^{18} \mathrm{ions} / \mathrm{cm}^{2}(400$ s). (D) The corresponding size distributions of the dot diameters are extracted from the images. The dotted lines represent Gaussian fits to the dot diameter histograms. Adapted from Facsko et al. (1999). 

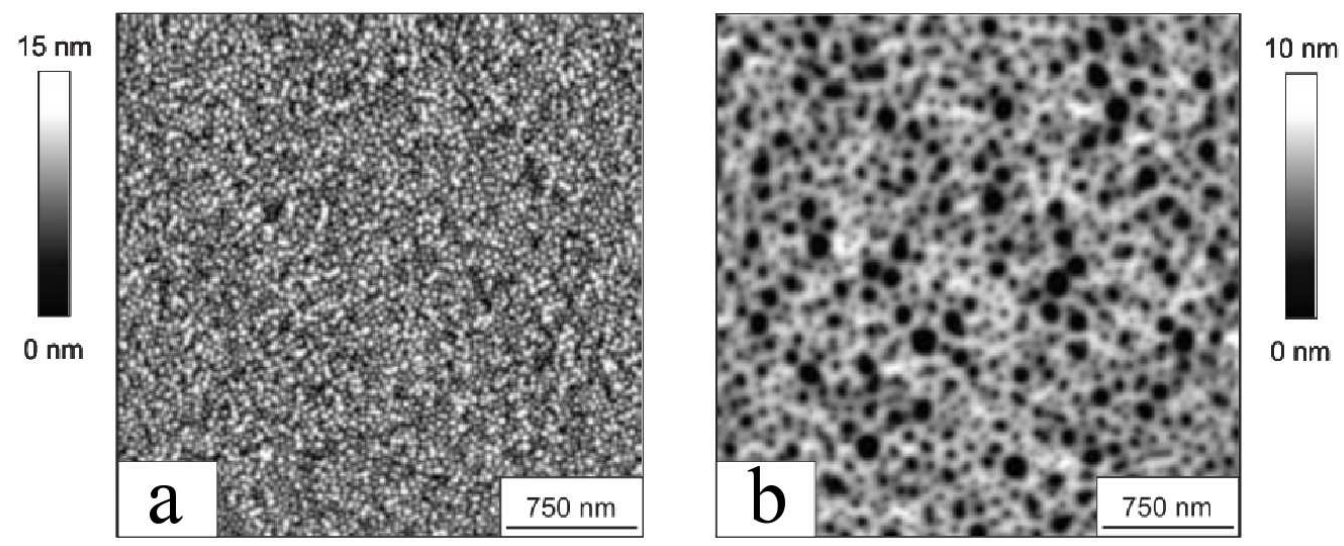

Figure 2.6: Silicon surface topographies after 20 min bombardment by $500 \mathrm{eV} \mathrm{Ar}^{+}$ionbeam (sample rotation), flux $=300 \mu \mathrm{A} / \mathrm{cm}^{2}, \theta$ (a) $0^{\circ}$ and (b) $75^{\circ}$. Cellular structures form in both cases. Adapted from Frost et al. (2004).

suppress the destabilizing effects of BH theory. This will be discussed in more detail in section. 3.1. The rotation frequency may have an important role in achieving the smoothed surfaces, similar to the formation of cellular structures. This will be discussed in section. 7.5 as well.

\subsection{Patterns on crystalline substrates}

The above mentioned characteristics are not commonly observed in experiments with metallic substrates. For example, ripples may form in normal incidence experiments or isotropic patterns in oblique incidence experiments may evolve without rotation . For a comprehensive collection of experimental results on metallic substrate see Valbusa et al. (2002). The different scenarios of pattern formation on single crystalline metallic substrates are mainly due to the different energy barriers in conjunction with the crystallographic anisotropies in such materials. On the other hand, Surface diffusion is not isotropic in crystalline substrates and the Sigmund's theory of sputtering neglects effects like channeling, which are due to the regular anisotropic structure. Valbusa et al. defined a "diffusive" and an "erosive" regime, in which the orientation of structures are determined by the unit cell direction or by the direction of the ion-beam, respectively. The diffusion rate is mainly controlled by the 

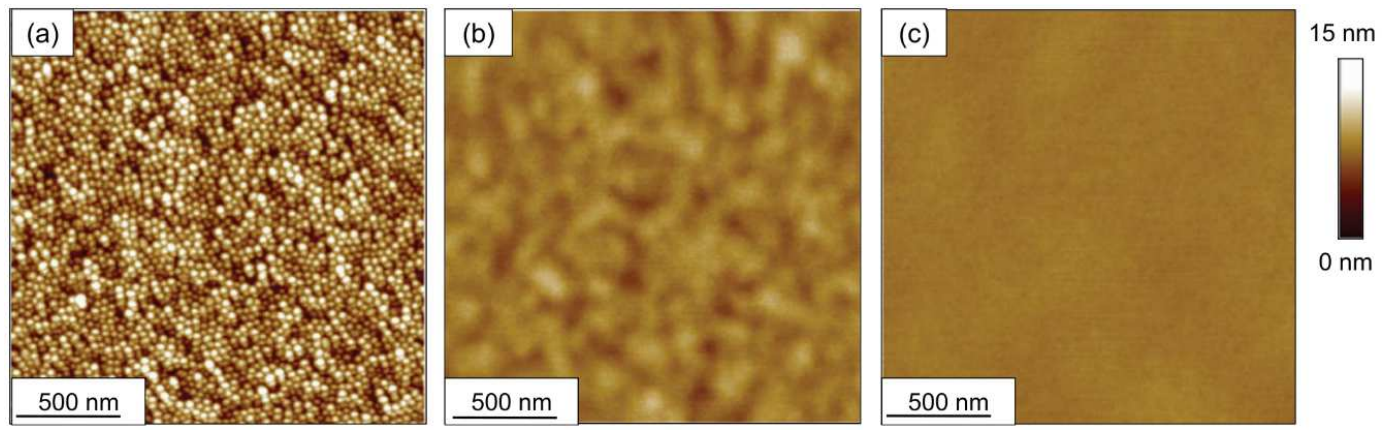

Figure 2.7: Sequence of AFM images which shows the progressive smoothing of a $\mathrm{Si}$ surface under $500 \mathrm{eV} \mathrm{Ar}{ }^{+}$ion-beam erosion, $\theta=45^{\circ}$, flux $=300 \mu \mathrm{A} / \mathrm{cm}^{2}$ (simultaneous sample rotation). (a) Initial surface (pre-roughened by $\mathrm{Ar}^{+}$erosion at $75^{\circ}$ ion incidence), (b) after $10 \mathrm{~min}$ (corresponding to a total applied ion fluence of $1.1 \times 10^{18}$ ions $/ \mathrm{cm}^{2}$ ) and (c) after $180 \mathrm{~min}\left(2.0 \times 10^{19} \mathrm{ions} / \mathrm{cm}^{2}\right)$. The rms roughness was reduced from $R_{q}=2.25$ $\mathrm{nm}$ to $R_{q}<0.2 \mathrm{~nm}$. Adapted from Frost et al. (2009).

substrate temperature and the erosion rate can be tuned by the ion-beam parameters. In Fig. 2.8, a transition from diffusive regime to erosive regime by increasing the temperature at fixed ion-beam parameters is depicted.

The pyramid-like structures formed in the diffusive regime are similar to the patterns which form in molecular beam epitaxy (MBE). Here, the difference to MBE is that deposition of adatoms is replaced by creation of vacancies by erosion. The main destabilizing factor in both cases is the biased diffusion of surface defects (adatoms or vacancies). Therefore, most of the basic features of patterns are similar in both MBE and IBS at high temperature and low flux regime. In the erosive regime, however, the main underlying process is the $\mathrm{BH}$ instability and diffusion is not the leading factor in the pattern formation. Hence, the main characteristics of patterns in the erosive regime are similar to those observed in the experiments on amorphized semiconductors (see section 4.2.4).

\subsection{Advanced patterning methods}

In the last few years, steps toward alternative complex IBS experiments have been taken. Some of these advanced methods are surfactant sputtering, sput- 

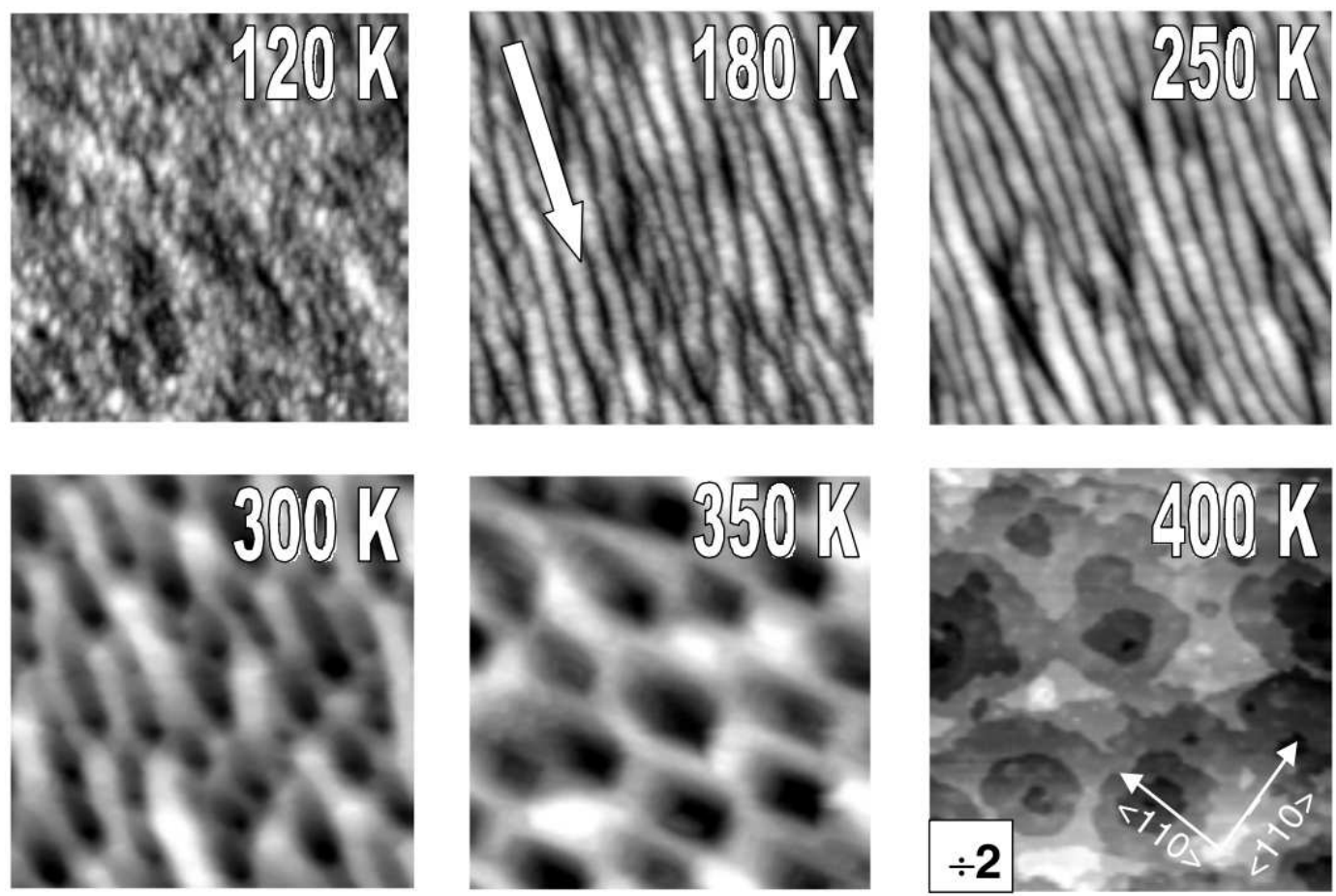

Figure 2.8: The role of surface temperature in the transition from the diffusive to the erosive sputtering regime for $\mathrm{Ag}(001) .1 \mathrm{keV} \mathrm{Ne}{ }^{+}$ions, $\theta=70^{\circ}$, flux $=2.2 \mu \mathrm{A} \mathrm{cm}^{2}, t=20$ min. The white arrow shows the ion-beam scattering plane. Image size $180 \times 180 \mathrm{~nm}^{2} ;$ at $T=400 \mathrm{~K}: 360 \times 360 \mathrm{~nm}^{2}$. Adapted from Valbusa et al. (2002). 
tering of alloys, sputtering of thin deposited films, sputtering with double or multiple beams, sputtering of substrate previously structured on micrometer length scales, etc. Apart from the practical advantages, exploring this unknown area of ion-beam sputtering technology poses new physical questions, which can be answered only by extending and developing the present theoretical models.

\subsubsection{Surfactant sputtering}

In surfactant sputtering, ion-beam erosion is accompanied by deposition of a second ("surfactant") atomic species (typically by co-sputtering a nearby metallic target). Co-deposition is adjusted in a way that a steady state coverage of (sub-) mono-layer thickness emerges.

Surfactant sputtering has been introduced by Hofsäss and Zhang (2008) as a novel method with a wide range of controllable pattern formation scenarios. The potentially wide spectrum of the applications of surfactant sputtering has not yet been probed. One of the available examples, shown in Fig. 2.9, is an arrangement of nano-drops of $\mathrm{Ag}$ on rippled Si substrate.

An important physical mechanism, which influences pattern formation is that the presence of metallic atoms on the surface of a substrate can considerably change the erosion rate of substrate atoms. Furthermore, the particular form of surface diffusion of metallic atoms and their tendency to mix or demix with the substrate can also affect the pattern formation. More details are presented in chapter 5 ,

\subsubsection{Compound beams}

Joe et al. (2009) performed experiments applying multiple beams (particularly dual-beams) and also sequential sputtering from different directions. The aim of these experiments was to produce structures of superimposed ripples formed in different directions. Although in none of the cases a linear superposition was observed, the case of dual-beams leads to square symmetric patterns (see Fig. (2.10). In the sequential sputtering of pre-structured surfaces, the stepwise rotation of the sample by $90^{\circ}$ led to a fast destruction of initially formed patterns before the new generation of patterns has grown in the perpendicular 

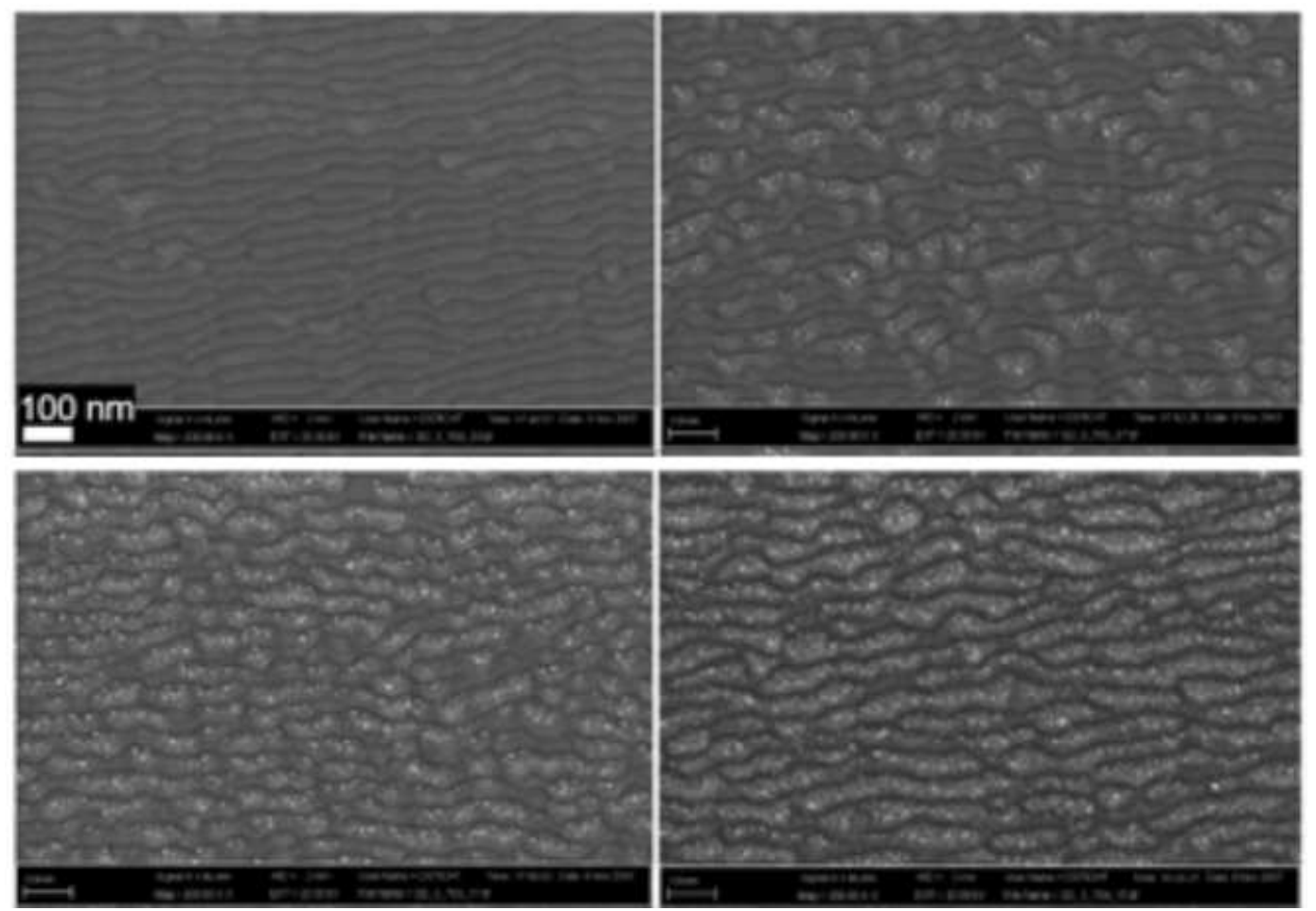

Figure 2.9: SEM pictures of Si surfaces eroded with $5 \mathrm{keV}$ Xe at $\theta=70^{\circ}$ and fluence $3 \times 10^{16}$ ion $/ \mathrm{cm}^{2}$ with $\mathrm{Ag}$ surfactants with different coverages (increasing from left to right and top to bottom) of up to $10^{16} \mathrm{Ag}$ atoms $/ \mathrm{cm}^{2}$. The ripple pattern and ripple wavelength for different coverages are strongly influenced by the surfactants. Ag nanoparticles of size $10 \mathrm{~nm}$ or less can be seen on the tops of the flat ripple plateaus. Adapted from Hofsäss and Zhang (2008). 

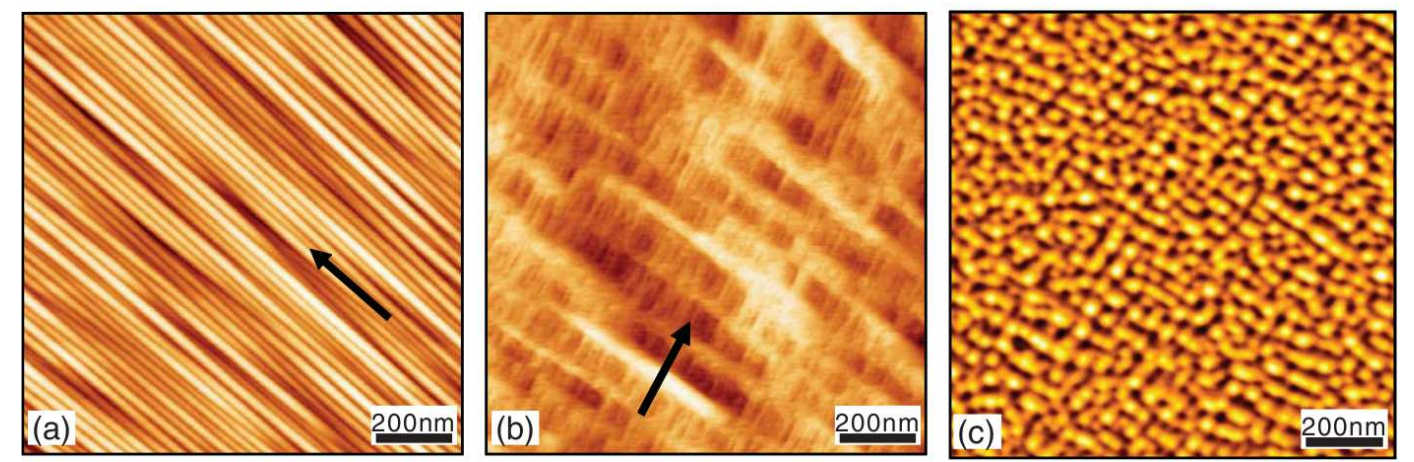

Figure 2.10: (a) Rippled $\mathrm{Au}(001)$ surface sputtered in the erosive regime, (b) Surface morphology induced by sequentially sputtering of the pre-rippled $\mathrm{Au}(001)$ with $2 \mathrm{keV} \mathrm{Ar}^{+}$ with flux $=0.31$ ions $/ \mathrm{nm}^{2} \mathrm{~s}$, fluence $=84.8 \mathrm{ions} / \mathrm{nm}^{2}$ and $\theta=72^{\circ}$. The initial ripple pattern is heavily damaged such that its order and mean coherence length are severely degraded. (c) Nano-patterns formed by dual ion-beam sputtering with flux $=3.25$ ions $/ \mathrm{nm}^{2} \mathrm{~s}$, fluence $=6350$ ions $/ \mathrm{nm}^{2}$ and $\theta=73^{\circ}$. Arrows indicate ion-beam projection. Adapted from Joe et al. (2009).

direction. More details are presented in section 7.4. 


\section{Chapter 3}

\section{Methods}

Pattern formation in IBS, can be studied theoretically at different levels and time-, energy- and length-scales with different approaches. The complete problem of IBS covers length scale from atomic sizes to some micrometers and time scales cover a range from $\sim 10^{-17} \mathrm{sec}$ (duration of the primary atom-ion collisions) to $\sim 10 \mathrm{~min}$ (typical pattern formation time scale). The same extension exists also in the covered range of energies; The upper bound is the energy of an impact, initiated with some $\mathrm{keV}$ ion and the lower bound is the energy involved in diffusion process at room temperature, i.e. the meV range. To cover this wide range of scales, different methods and approaches must be applied and at different scales different approximations are necessary. A combination of all the approaches presents a multi-scale picture of the whole phenomenon. In this chapter we introduce the following theoretical models which we apply to IBS:

(i) Atomistic simulations based on binary collision approximation (BCA): In this class of simulations, one starts from single impacts of ions on the surface and follows cascades of atomic collisions, aiming to provide a statistics of sputtering effects caused by impinging ions. The typical lengthscales considered in this approach range from some $\AA$ to some $\mathrm{nm}$.

(ii) Kinetic theory: In this approach one studies the evolution of the phase space density $f(\mathbf{r}, \mathbf{p}, t)$ of displaced atoms (and of the ions) at position $\mathbf{r}$ and with momentum $\mathbf{p}$ in the framework of Boltzmann's transport theory.

(iii) Kinetic Monte Carlo simulations: Many results of this thesis are obtained from this method. Usually, one starts from the results of kinetic 
theory, i.e. one uses a simple functional form of the averaged energy deposited by a collision cascade for single ion impact to determine the erosion probability of surface atoms. Diffusion processes can easily be added in this approach. Simulating larger scales of some hundreds of $\mathrm{nm}$ can be studied by KMC.

(iv) Continuum theory of surface evolution: In the continuum model of surface evolution the height of surface is considered as a continuous, single valued smooth function of plane coordinates $h(x, y)$. In the most general form, the time evolution of $h$ is described by a non-linear stochastic partial differential equation, the growth equation. In principle the growth equation may include all the underlying processes which lead to the surface evolution and also external noise by randomly shot ions. Here, length scales larger than the penetration depth and atomistic sizes are considered and therefore small size fluctuations are neglected. The main parts of continuum theories are erosion and surface diffusion. Most of the theories in this framework are based on the Sigmund's theory of sputtering and a thermally activated diffusion model.

\subsection{Binary collision approximation}

The most microscopic approach to pattern formation under IBS would be a full-fledged Molecular Dynamics (MD) simulation. However such a simulation has to bridge the above-mentioned scales in time, space and energy, which is not possible at present. Nevertheless, the approach is used for single ion impacts to study the defect generation and also the mass transport due to displaced atoms. But an approximate version of MD, the binary collision approximation (BCA), has become a versatile tool in the study of ion sputtering phenomena, including pattern formation. The main idea of this method is to reduce all interactions to a series of binary collisions between pairs of particles. In between collisions, the trajectory of the particles are straight-line segments traversed with constant velocity, initiating from a collision and ending at the next collision. Changes in velocity and position after each collision can be integrated numerically (Robinson and Torrens 1974; Robinson 1994). This approach is successfully used to quantitatively calculate sputtering yields in the much used and well established programs TRIM and SRIM. Koponen et al. (1997) have also used it to study the formation of rip- 
(a)

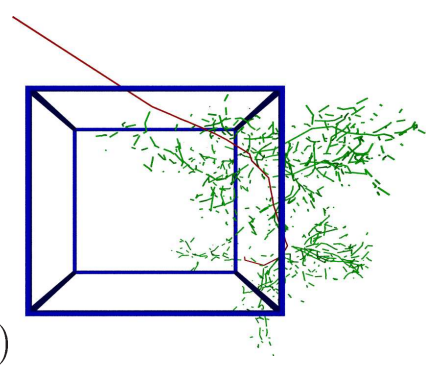

(b)

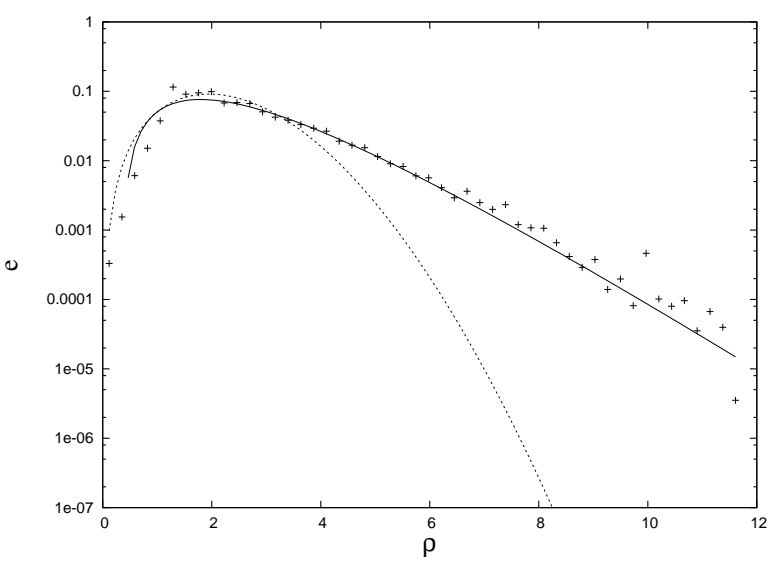

Figure 3.1: (a) Sample cascade originating from an impact of a $5 \mathrm{keV} \mathrm{Cu}$ ion on a $\mathrm{Cu}$ crystal. The angle of incidence is $60^{\circ}$. The cube shown, acts just as scale and has volume 2.65 $\mathrm{nm}^{3}$. (b) Surface density of mean energy of sputtered $\mathrm{Cu}$ atoms vs. distance $\rho$ (measured in units of $a=3.61 \AA$ ) from point of ion incidence. The solid line is the best fit to the data; $0.297\left(\rho^{2}-0.392 \rho\right) \exp (-1.27 \rho)$ and the dotted line, which corresponds to a Gaussian fit, is obviously inadequate. Adapted from Feix et al. (2005).

ples under IBS. Feix (2002) and Feix et al. (2005) have studied the sputtering of $\mathrm{Cu}$ crystals by means of BCA simulations to test some assumptions and approximations used in less microscopic approaches.

\subsubsection{Cascade shape}

Feix et al. (2005) considered an ensemble of 6000 impinging ions and calculated the averaged statistics of the induced collision cascades (see Fig. 3.1 (a)). One of the main results is about the distribution of deposited energy by a single ion as this quantity underlies the continuum theory and the KMC approach (see below). For normal incidence, the simulations show an energy distribution as depicted in Fig. 3.1(b), which has a minimum near the position where the ion penetrates the surface, and decays exponentially with distance. This from deviates significantly from Gaussian shape centered at the location of primary knock-on collision, which is used in the vast majority of continuum and KMC approaches, and was proposed by Sigmund (1969) on the basis of an approximate treatment of the kinetic theory. 


\subsubsection{Defect generation}

Apart from erosion of the substrate atoms by the energy transferred from ions, generation of surface defects (adatoms and vacancies) is known to be another effect induced by collision cascades (Nordlund et al. 1998). Excited atoms reaching the surface with energies more than the surface binding energy $E_{b}$, will be sputtered off, and leave a vacancy, however a large fraction of particles have energies less than $E_{b}$. These atoms remain on the surface and become adatoms. At high temperature, defects recombine and vanish rapidly, whereas at low temperature regime a large number of them remains for longer time (Floro et al. 1995). Feix (2002) found a distribution of the energy of the particles reaching the surface $\epsilon_{s}$, which obeys a simple power low

$$
p\left(\epsilon_{s}\right) \approx \frac{a}{\left(b+\epsilon_{s}\right)^{\gamma}} \sim \epsilon_{s}^{-2}
$$

with $a=5.26, b=5.03$ and $\gamma=1.87$ for $5 \mathrm{keV} \mathrm{Cu}$ ion hitting a $\mathrm{Cu}$ target. The shape of the distribution is almost independent of the distance from the impact point up to a large distance (see Fig. 3.2). This finding is in accordance with experimental observations and a simple theory of cascades by Farmery and Thompson (1968).

\subsubsection{Down-hill current}

Carter and Vishnyakov (1996) observed that in off-normal incidence (up to $\left.45^{\circ}\right)$ sputtering of $\mathrm{Si}$ with high energy $(10-40 \mathrm{keV}) \mathrm{Xe}^{+}$ions, sputtering erosion can induce smoothing. Using MD simulations Moseler et al. (2005) explained the irradiation-induced smoothing on diamond-like carbon surfaces. They found a down-hill current of atoms along the beam-direction induced by the ions. This current may suppress the curvature dependent BH instability (section 2.1.1), because they transport atoms back to the ripple valleys. The down-hill current is proportional to the surface slope $\mathbf{j} \propto-\nabla h$. By substitution of the current density into the continuity equation $\partial h / \partial t=-\nabla \cdot \mathbf{j}$, one obtains a smoothing equation for the height field $h, \partial h / \partial t \propto \nabla^{2} h$.

This down-hill current is also easily observed in BCA as demonstrated in Fig. 3.3. 


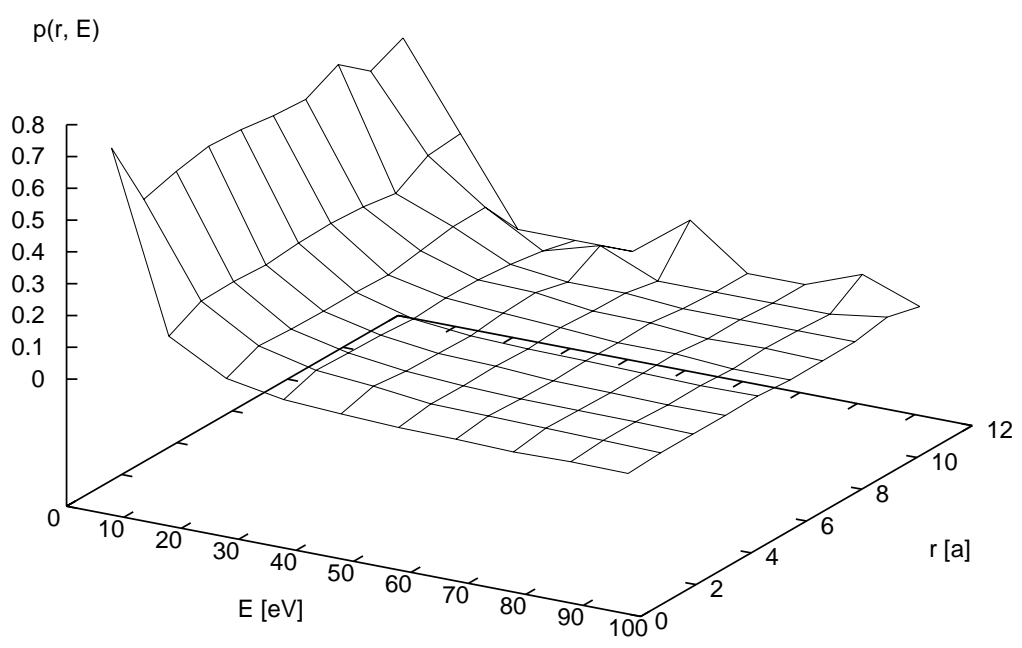

Figure 3.2: Energy distribution extracted from 6000 independent $5 \mathrm{keV}$ impacting $\mathrm{Cu}$ ions for different distances from impact point (measured in units of $a=3.61 \AA$ ). The distribution is almost independent of $\mathbf{r}$. Adapted from Feix (2002). 


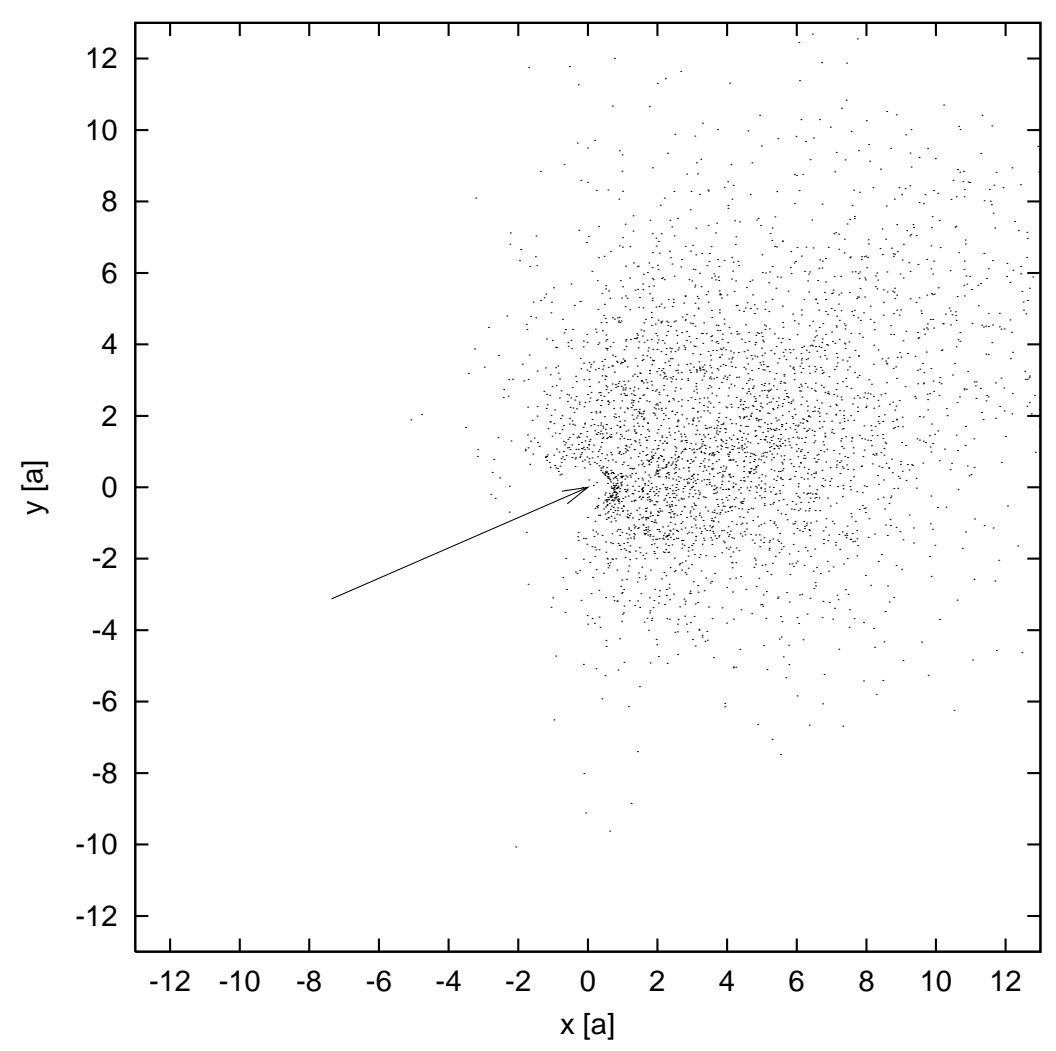

Figure 3.3: Spatial distribution of ejected $\mathrm{Cu}$ atoms emerging from 6000 independent trials of hitting the $(\mathrm{x}, \mathrm{y})$ crystal surface (oriented in (100) direction) with a single $5 \mathrm{keV}$ $\mathrm{Cu}$ ion at oblique incidence. Distances are measured in units of $a=3.61 \AA$. Adapted from Feix (2002). 


\subsection{Kinetic theory}

To obtain statistics of the collision cascade and calculate the sputtering yield depending on ion-target parameters, Sigmund $(1969 ; 1973)$ presented a solution of a Boltzmann transport equation with assumption of random slowing down of ions in an infinite medium. The main approximation in this approach is the neglect of interactions between atoms, which thus form a tree of non-interacting particles (referred to as the "collision cascade"). The most important results, which underlie the approaches in subsequent scales are the following:

(i) The erosion rate at each surface point is proportional to the power brought to this point via collision cascades.

(ii) The distribution of collision cascades is calculated approximately. Sigmund found that in some cases this form can be approximated by a simple Gaussian.

(iii) The scattering events originated by the penetration of energetic ions, leading to sputtering occur in a layer near to the surface with very small thickness. Most of the sputtered atoms belong to a thin surface layer $(\sim 5 \AA)$.

(iv) The energy distribution of ejected particles follows Fig. 3.1, This result was first obtained by an elementary argument on cascades by Thompson (1968).

These results then became the main principles of almost all later theoretical works on IBS.

\subsection{Kinetic Monte Carlo}

All the existing KMC simulation models of IBS (for examples see Chason et al. (2006), Stepanova and Dew (2006), and Hartmann et al. (2002)), are based upon the results from the kinetic theory, mentioned above and include two parts of erosion, upon Sigmund's theory and a surface relaxation process. A simulation run consist of a sequence of single ion shots, a calculation of the deposited energy at the current surface for each ion and random discrete hopping of surface atoms corresponding to surface diffusion. Most models (with the exception of the work by (Bartosz Liedke 2009) ) describe the surface in the framework of a solid-on-solid (SOS) model, thus excluding overhangs, drops and bulk vacancies. Furthermore, a re-deposition of sputtered particles is not taken into account. In the following we describe some details of the models, 
which was developed from Hartmann et al. (2002) to Hartmann et al. (2009) (HKGK model).

The system consist of a square lattices of size $L \times L$ (with periodic boundary conditions, if not stated otherwise) and the SOS surface is described by an integer-valued time-dependent height function $h(x, y, t)$ on the lattice. In most cases, we start from a flat surface, i.e. $h(x, y, 0)=0$. The details of erosion and diffusion trials are as the following.

\subsubsection{Erosion}

As mentioned above, the erosion process is based on Sigmund's theory, i.e. the Sigmund formula Eq. 3.4 is applied for every single impinging ion. An ion starts at a random position in a plane parallel to the plane of the initially flat surface $(x-y$ plane), and follows a straight trajectory inclined at angle $\theta$ to the normal of this plane. The ion penetrates into the solid through a length $a$ and releases its energy. Then we check all the lateral atoms as the subject for sputtering such that an atom at a position $\mathbf{r}=(x, y, h)$ is eroded with probability proportional to $E(\mathbf{r})$.

We have put $\epsilon$ to be $(2 \pi)^{3 / 2} \sigma \mu^{2}$, which leads to sputtering yields $Y \simeq 7.0$, thus should be kept in mind when comparing simulation results to experimental data. According to the Bradley Harper theory, the ripple wavelength $\lambda$ scales like $\lambda \sim Y^{-1 / 2}$ so that lower yields lead to correspondingly larger length scales.

Throughout this work we use a set of parameters as default values if not stated otherwise. We fixed $\sigma=3, \mu=1.5$ and $a=9.3$ (in lattice constant).

\subsubsection{Diffusion}

We have implemented different models to describe the surface motion of atoms. These range from simple, irreversible surface relaxation to activated hopping over energy barriers, which may depend both on initial and final state of the move and include Ehrlich-Schwoebel non-equilibrium kinetic effects. We always use "full" diffusion models, so one diffusion step refers to a complete sweep of the lattice. In the following, we briefly introduce the three basic types of diffusion models, which we have used throughout our simulations. Details of the different models and their effects on pattern formation are discussed in 
section 4.2 .

(i) A simple, non-thermal, irreversible relaxation process has been introduced by Wolf and Villain (1990). For each column, it is tested once during a sweep, whether the particle at the top of the column can increase its coordination number, i.e. its number of nearest neighbors, by hopping to a neighboring column. If this is the case, the particle hops to that neighbor column where it obtains the highest coordination number (section 4.2.1).

(ii) A class of thermal diffusion models is based upon a Hamiltonian $\mathcal{H}$, which controls the thermal roughening of a facet. Trial moves from site $i$ to a nearest neighbor site $f\left(h_{i} \rightarrow h_{i}-1, h_{f} \rightarrow h_{f}-1\right)$ are accepted with probability $\left.p(i \rightarrow f)=\left[1+\exp \left(\Delta \mathcal{H} / k_{\mathrm{B}} T\right)\right)\right]^{-1}$ where $\Delta \mathcal{H}$ is the change in Hamiltonian due to the hop. (section 4.2.2).

(iii) The "Arrhenius" models are based on a kinetic procedure and use hopping via transition states. For each step, a move from initial $(i)$ to final $(f)$ configuration is chosen randomly from a predefined list. Here we restrict moves to nearest neighbor hops from site $i$ to site $f$. We would have to include more moves, if we want to model material specific diffusion processes. The move is performed with a probability proportional to an Arrhenius hopping rate

$$
k=k_{0} \exp \left(-\frac{E(i \rightarrow f)}{k_{B} T}\right)
$$

Values of the energy barriers $E(i \rightarrow f)$ have to be taken from experimental or simulation data (section 4.2.3).

The connection between time in KMC models and real experiments is made by comparing the attempt frequencies of different events in KMC with corresponding kinetic rates in the lab condition. In our model there are two different time scales, (i) the time intervals between the shooting of two impinging ions $\tau_{i}$ and (ii) the waiting time between diffusion sweeps $\tau_{d}$. By tuning these two time scales, a wide range of experimental conditions can be covered. Our default values correspond to a typical flux of 0.75 (ion/atom second) and a temperature of $350 \mathrm{~K}$. 


\subsection{Continuum theory}

\subsubsection{Bradley-Harper model}

Bradley and Harper started from the results of kinetic theory, that the normal velocity of the eroded surface

$$
\frac{\partial h(x, y, t)}{\partial t} \frac{1}{\sqrt{1+(\nabla h)^{2}}}=-v_{n}(x, y, t)
$$

is proportional to the total energy transferred to the point $(x, y, h(x, y))$ by the collision cascades. An arbitrary ion impinging the surface at point $P$, comes to rest at point $O^{\prime}$ after penetrating into the solid by a distance of $a$ along its trajectory. The deposited energy of the ion at any point $O$ at the surface is a function of the distance vector $\mathbf{R}=(X, Y, Z)$ between $O$ and $O^{\prime}$. The averaged energy deposition function is taken to be a Gaussian

$$
E(\mathbf{R})=\frac{1}{(2 \pi)^{3 / 2} \sigma \mu^{2}} \exp \left(-\frac{X^{2}+Y^{2}}{2 \mu^{2}}-\frac{Z^{2}}{2 \sigma^{2}}\right)
$$

as proposed by Sigmund (1969). $\mu$ and $\sigma$ are width of Gaussian function parallel and perpendicular to the beam trajectory. To calculate the erosion rate, all the contributions from homogeneously impinging ions at incidence angle $\theta$ with respect to the normal of the surface should be summed up;

$$
v_{n}(\mathbf{r})=Y J_{i o n} \int \mathrm{d} \mathbf{r}^{\prime} \mathrm{E}\left(\mathbf{r}-\mathbf{r}^{\prime}\right) \hat{\mathrm{n}} \cdot \mathbf{e}_{\theta}
$$

where $J_{\text {ion }} \mathbf{e}_{\theta}$ is the ion flux with

$$
\mathbf{e}_{\theta}=\left(\begin{array}{c}
\sin (\theta) \\
0 \\
\cos (\theta)
\end{array}\right)
$$

$\hat{n}$ is the unit vector normal to the surface and $Y$ is the sputter yield. The integral is taken over the surface. The integral is evaluated in a gradient expansion (i.e. in $(\nabla h)^{n}$ ) and a small slope approximation which starts with the following terms:

$$
\frac{\partial h(x, y, t)}{\partial t}=-v_{0}(\theta)+v_{0}^{\prime}(\theta) \frac{\partial h(x, y, t)}{\partial x}+\nu_{x} \frac{\partial^{2} h(x, y, t)}{\partial x^{2}}+\nu_{y} \frac{\partial^{2} h(x, y, t)}{\partial y^{2}}
$$


$v_{0}$ is the average erosion velocity of a planar surface. $\nu_{x}$ and $\nu_{x}$ are "effective surface tensions" in directions parallel and perpendicular to the projected direction of ion-beam onto the surface. To solve the obtained growth equation, we let $h(x, y, 0)=A \exp \left[i\left(k_{x} x+k_{y} y\right)\right]$. The general solution of Eq. 3.6 for the mode $\mathbf{k}=\left(k_{x}, k_{y}\right)$ takes on the from

$$
h_{\mathbf{k}}(x, y, t)=-v_{0}(\theta) t+A \exp \left[i\left(k_{x} x+k_{y} y-\omega t\right)+\Gamma t\right] .
$$

substitution of the solutions leads to

$$
\omega=-v_{0}^{\prime}(\theta) k_{x}
$$

and

$$
\Gamma\left(k_{x}, k_{y}\right)=-\nu_{x} k_{x}^{2}-\nu_{y} k_{y}^{2}
$$

This means that an arbitrary mode $\mathbf{k}$ propagate along the orientation of the beam (projected onto the $x-y$ surface) with phase velocity $-v_{0}^{\prime}$ and also grows (decays) in amplitude with the rate $\Gamma$. The $\theta$ dependence of effective surface tensions results from the gradient expansion and one example is shown in Fig. 3.4 for the default parameters of our KMC simulation. For some values of $\theta$, both $\nu_{x}$ and $\nu_{y}$ are negative, leading to positive growth rate $\Gamma$ for all wavevectors. In experiments, it is observed that a specific wavelength grows faster than all others and forms periodic ripple-like structures. A stabilizing, i.e. smoothing mechanism, which is lacking in Eq. 3.6 is surface diffusion, which gives rise to a term $\propto \nabla^{4} h$ (see section 4.2). Adding this term results in linear evolution equation of Bradley-Harper theory,

$\frac{\partial h(x, y, t)}{\partial t}=-v_{0}(\theta)+v_{0}^{\prime}(\theta) \frac{\partial h(x, y, t)}{\partial x}+\nu_{x} \frac{\partial^{2} h(x, y, t)}{\partial x^{2}}+\nu_{y} \frac{\partial^{2} h(x, y, t)}{\partial y^{2}}-B \nabla^{2} \nabla^{2} h$

where $B$ is the coefficient of surface diffusivity. Taking the diffusion mechanism into account changes the growth rate into

$$
\Gamma\left(k_{x}, k_{y}\right)=-v_{x} k_{x}^{2}-v_{y} k_{y}^{2}-B\left(k_{x}^{2}+k_{y}^{2}\right)^{2} .
$$

Now for any value of $\theta$ (except $\theta=0$ and $\theta=\theta_{c}$ where $\nu_{x}=\nu_{y}$ ), $\Gamma$ has a maximum value for a single $\left(k_{x}^{2}, k_{y}^{2}\right)$. Since the included diffusion term is isotropic, the maximum of $\Gamma$ occurs always for $\mathbf{k}$ which is either in $x$ or $y$ direction, i.e. $\mathbf{k}=\left(k_{x}^{\max }, 0\right)$ or $\mathbf{k}\left(0, k_{y}^{\max }\right)$. The maximum lies in the direction, for which 


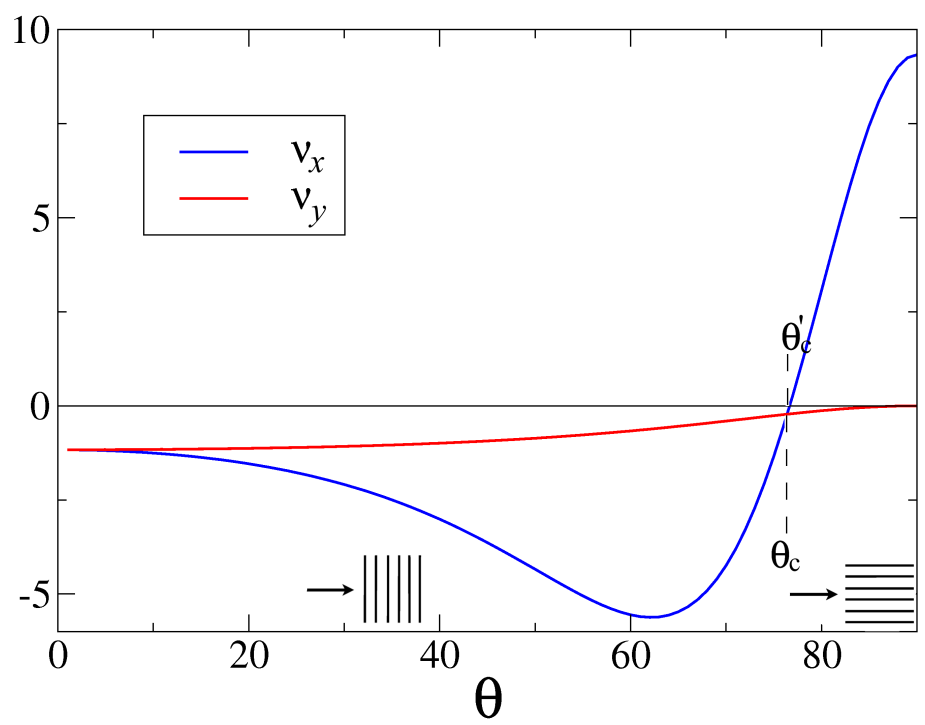

Figure 3.4: Effective surface tensions in two directions, parallel and perpendicular to the ion-beam direction as a function of incidence angle $\theta$ for $\sigma=3 \mu=1.5$ and $a=9.33$. For $0<\theta<\theta_{c}, \nu_{x}, \nu_{y}<0$ and $\left|\nu_{x}\right|>\left|\nu_{y}\right|$, therefore, the growth of instabilities with the wavevectors parallel to the beam direction is faster and ripples perpendicular to the beam direction form. For $\theta_{c}<\theta<\theta_{c^{\prime}},\left|\nu_{y}\right|>\left|\nu_{x}\right|$ and therefore ripples parallel to the ion-beam direction form. For $\theta_{c^{\prime}}<\theta, \nu_{x}$ becomes positive and perturbations with the wavevector in $x$ direction damp and again formation of the ripples parallel to the beam direction expected.

the negative surface tension has the larger negative value. This predicts for a wide range of materials and ion parameters, ripples with wavevector aligned parallel to the projection of ion-beam for $\theta<\theta_{c}$ and ripples with wavevector perpendicular to the ion-beam projection for $\theta>\theta_{c}$. This prediction has been confirmed in numerous experiments and makes the BH theory reliable for surface texturing by ion-beam. The typical length scale of patterns predicted by linear theory of $\mathrm{BH}$ is

$$
\ell=(2 \pi) \sqrt{\frac{2 B}{\left|\nu_{m}\right|}}
$$

where $\nu_{m}=\min \left[\nu_{x}, \nu_{y}\right]$.

\subsubsection{Cuerno-Barabási non-linear model}

Cuerno and Barabási (1995) continued the small slope expansion and includes 
non-linear corrections to the Eq. 3.3 They also took into account the "shot noise" i.e., the random arrival of ions to the surface as a Gaussian white noise $\eta(x, y, t)$ with zero mean and variance proportional to the flux. The growth equation then becomes

$\frac{\partial h(x, y, t)}{\partial t}=-v_{0}(\theta)+v_{0}^{\prime}(\theta) \frac{\partial h}{\partial x}+\nu_{x} \frac{\partial^{2} h}{\partial x^{2}}+\nu_{y} \frac{\partial^{2} h}{\partial y^{2}}+\frac{\lambda_{x}}{2}\left(\frac{\partial h}{\partial x}\right)^{2}+\frac{\lambda_{y}}{2}\left(\frac{\partial h}{\partial y}\right)^{2}-B \nabla^{2}\left(\nabla^{2} h\right)+\eta$.

This equation is an anisotropic version of the Kuramuto-Sivashinsky (KS) equation, which is well known in pattern formation theories (Kuramoto and Tsuzuki 1976; Sivashinsky 1977).

A crossover time $t_{c}$ is defined as the time in which the nonlinear effects become dominant and the system leaves the validity region of the linear approximation. From the linear equation, the amplitude of ripples at $t_{c}$ is $\sim \exp \left(\left|\nu_{m}\right| t_{c} / \ell^{2}\right)$, whereas from $\partial_{t} h \sim \lambda(\nabla h)^{2}$ the amplitude is estimated in order of $\ell^{2} / \lambda t_{c}$. Combining these two relations, the crossover time is

$$
t_{c} \sim\left(\frac{B}{\nu_{m}^{2}}\right) \ln \left(\frac{\left|\nu_{m}\right|}{\lambda}\right)
$$

Depending on the signs of $\nu_{x}, \nu_{y}, \lambda_{x}$ and $\lambda_{y}$, different morphologies are expected from non-linear theory. Typically for small values of $\theta$ where $\nu_{x}$ and $\nu_{y}$ are both negative, $\lambda_{x}$ and $\lambda_{y}$ are also negative. For short time scales $\left(t \ll t_{c}\right)$, the same ripples as predicted by $\mathrm{BH}$ form, but ripples get blurred and disappear gradually for long times $\left(t \gg t_{c}\right)$. The patterns show the typical Kuramoto-Sivashinsky type of spatio-temporal chaos. Increasing the incidence angle, $\lambda_{x}$ and $\lambda_{y}$ obtain different signs where $\nu_{x}$ and $\nu_{y}$ are still both negative. Park et al. (1999) have shown that two transitions occur in this regime. In early stage of pattern formation, standard ripples from linear theory form; At the first transition, ripples disappear and the surface becomes rough; At the second transition, stable ripples with rotated orientation by an angle of $\tan ^{-1} \sqrt{-\lambda_{x} / \lambda_{y}}$ form. The stability of these ripples can be understood as a consequence of a non-linear cancellation of modes. (Rost and Krug 1995).

\subsubsection{Makeev, Cuerno and Barabási model}

Makeev et al. (2002) have continued the gradient expansion, including 4th or- 
der derivatives.

$$
\hat{D}=-D_{x x} \frac{\partial^{4} h}{\partial x^{4}}-D_{y y} \frac{\partial^{4} h}{\partial y^{4}}-D_{x y} \frac{\partial^{4} h}{\partial x^{2} \partial y^{2}} .
$$

These terms resemble (anisotropic) a new type of surface diffusion. They have been called "erosion-induced diffusion", although they does not describe a real transport process (and may in fact lead to roughening as well as smoothing). We want to point out that tempting interpretation of these terms as ioninduced diffusion can be misleading, even if they act as smoothing mechanism. To this end, in the next section, we study the linear stability analysis of the continuum theory without making use of the gradient expansions.

\subsubsection{Non-local linear stability analysis}

For simplicity, we restrict our analysis to the case of normal incidence $(\theta=0)$. We start from Eq. 3.5 and consider a broad class of energy deposition functions of the form

$$
E(x, y, z)=f(x, y) g(z)
$$

so that

$$
\partial_{t} h\left(\mathbf{r}_{1}\right)=-Y J_{i o n} \int f\left(x_{1}-x, y_{1}-y\right) g\left(h\left(x_{1}, y_{1}\right)-h(x, y)\right) d x d y
$$

$g\left(h(\mathbf{r})-h\left(\mathbf{r}_{1}\right)\right)=g(\Delta h)$ can be expanded around $\Delta h=0 ; g(\Delta h)=g(0)+$ $g^{\prime}(0) \Delta h$ the first term leads to a constant erosion velocity. In the coordinate system moving with this velocity,

$$
\partial_{t} h\left(\mathbf{r}_{1}\right)=-Y J_{i o n} \int f\left(\mathbf{r}-\mathbf{r}_{1}\right) g^{\prime}(0)\left(h(\mathbf{r})-h\left(\mathbf{r}_{1}\right)\right) d r^{2} .
$$

By changing the variables $\xi=\mathbf{r}-\mathbf{r}_{1}$, we have

$$
\partial_{t} h\left(\mathbf{r}_{1}\right)=-Y J_{\text {ion }} \int f(\xi) g^{\prime}(0)\left(h\left(\xi+\mathbf{r}_{1}\right)-h\left(\mathbf{r}_{1}\right)\right) d \xi^{2}
$$

We substitute $h$ by the inverse Fourier transform of the height profiles $\hat{h}(\mathbf{q})$

$$
\partial_{t} h\left(\mathbf{r}_{1}\right)=\frac{-Y J_{i o n}}{(2 \pi)^{2}} \int f(\xi) g^{\prime}(0) \int\left(e^{i \mathbf{q} \cdot\left(\xi+\mathbf{r}_{1}\right)}-e^{i \mathbf{q} \cdot \mathbf{r}_{1}}\right) \hat{h}(\mathbf{q}) d q^{2} d \xi^{2}
$$

Now we Fourier transform the whole equation

$\partial_{t} \int e^{-i \mathbf{k} \cdot \mathbf{r}_{1}} h\left(\mathbf{r}_{1}\right) d r_{1}^{2}=\frac{-Y J_{i o n}}{(2 \pi)^{2}} g^{\prime}(0) \iint e^{-i \mathbf{k} \cdot \mathbf{r}_{1}+i \mathbf{q} \cdot \mathbf{r}_{1}} \int f(\xi)\left(e^{i \mathbf{q} \xi}-1\right) \hat{h}(\mathbf{q}) d \xi^{2} d q^{2} d r_{1}^{2}$. 
Note that $\int e^{-i \mathbf{k} \cdot \mathbf{r}_{1}+i \mathbf{q} \cdot \mathbf{r}_{1}} d r_{1}^{2}$ gives a delta function $(2 \pi)^{2} \delta^{2}(\mathbf{k}-\mathbf{q})$, so

$\partial_{t} \hat{h}(\mathbf{k})=-Y J_{i o n} g^{\prime}(0) \int f(\xi)\left(e^{i \mathbf{k} \xi}-1\right) \hat{h}(\mathbf{k}) d \xi^{2}=-\mathcal{E} g^{\prime}(0) \hat{h}(\mathbf{k})(\hat{f}(\mathbf{k})-\hat{f}(\mathbf{k}=0))$.

This equation leads to instability/stability, if the sign of real part of the prefactor of $h(\hat{\mathbf{k}})$ at the right-hand-side is negative/positive.

For all functions $f(r)>0,(\hat{f}(\mathbf{k})-\hat{f}(\mathbf{k}=0))<01$ Therefore if $g^{\prime}(0)>0$ all the modes will be unstable and roughening happens in all length-scales.

Let us specialize to the Gaussian energy deposition function Eq 3.4 and put

$$
f(x, y)=\frac{1}{2 \pi \mu^{2}} \exp ^{-\frac{x^{2}+y^{2}}{2 \mu^{2}}} .
$$

Thus

$$
\hat{f}(\mathbf{k})=e^{-\frac{k^{2} \mu^{2}}{2}}
$$

and

$$
\partial_{t} \hat{h}(\mathbf{k})=-J_{i o n} Y\left(e^{-\frac{k^{2} \mu^{2}}{2}}-1\right) \hat{h}(\mathbf{k}) .
$$

If we now apply a gradient expansion up to 4th order, we get

$$
\partial_{t} \hat{h}(\mathbf{k})=-J_{\text {ion }} Y\left(-\frac{1}{2} k^{2} \mu^{2}+\frac{1}{8} k^{4} \mu^{4}-\mathcal{O}\left(k^{6}\right)\right) \hat{h}(\mathbf{k}) .
$$

Note that the $k^{4}$-term would lead to a stabilization of modes at short wavelength. This result, however, is spurious as all modes are unstable, if all terms of the gradient expansions are taken into account.

\subsection{Data analysis}

We use two main methods to analyze the data obtained from simulations, scaling analysis of surface roughness and power spectral density of the surface profile. They are introduced and explained in the following.

\subsubsection{Scaling analysis}

To study the scaling behavior of the growing surface, we investigate the interface "width" which is a measure for the "roughness" of the interface. We define the width of a 2-d interface discretized in $L \times L$ segments, at time $t$ as

\footnotetext{
${ }^{1}$ We want to evaluate $\Delta=(f(k)-f(k=0))$. By definition $\Delta=\int \exp (i k r) f(r) d r-$ $\int \exp (i 0 r) f(r) d r=\int \exp (i k r) f(r) d r-\int f(r) d r$. The real part of $\Delta, \Re(\Delta)=$ $\int_{r} \cos (k r) f(r) d r-\int_{r} f(r) d r$, is clearly negative.
} 


$$
w(L, t)=\sqrt{\frac{1}{L^{2}} \sum_{i=1}^{L} \sum_{j=1}^{L}[h(i, j, t)-\bar{h}(t)]^{2}}
$$

where $h(i, j, t)$ is the height of segment $(i, j)$ and $\bar{h}(t)$ is the average height of interface at time $t$. Starting with a flat surface, $h(i, j, 0)=0$ for all $i$.

For large classes of growth models $w(L, t)$ obeys a universal scaling from

$$
w(L, t) \sim L^{\alpha} f\left(\frac{t}{L^{z}}\right)
$$

where the scaling function $f$ has the following shape

$$
f(u) \sim u^{\beta}: u \ll 1 \quad f(u)=\text { const }: u \gg 1 .
$$

$\alpha, \beta$ and $z$ are called "roughness", "growth" and "dynamic" exponent. These exponents satisfy the scaling law $z=\alpha / \beta$. Based on the values of the scaling exponents, surface and interface growth mechanisms are classified into different universality classes. For more detail see Barabási and Stanley (1995).

\subsubsection{Power spectral density}

The power spectral density (PSD) of a given 2-d surface profile $h(x, y)$ is defined as

$$
S\left(q_{x}, q_{y}\right)=\left|\frac{1}{\sqrt{2 \pi}} \int_{-\infty}^{\infty} h(x, y) e^{-i\left(q_{x} x+q_{y} y\right)} d x d y\right|^{2}=\frac{\mathcal{F}(\vec{q}) \mathcal{F}^{*}(\vec{q})}{2 \pi}
$$

where $\mathcal{F}$ is Fourier transform of the function $h$ and $\mathcal{F}^{*}$ is its complex conjugate. The surface width is calculated by integration of $S$

$$
w^{2}(t)=\frac{1}{2 \pi} \int_{0}^{\infty} q S(q, t) d q
$$

For any given linear growth equation, one can consider plane wave solutions with the wavevector $\vec{q}=\left(q_{x}, q_{y}\right)$ satisfying a growth equation in the form of

$$
\frac{\partial h(\vec{q}, t)}{\partial t}=-h(\vec{q}, t) R(q)
$$

where $R(q)$ is the growth rate which can be positive or negative leading to roughening or smoothing the surface respectively. Eq. 3.32 can be easily solved and the corresponding power spectral density function is 

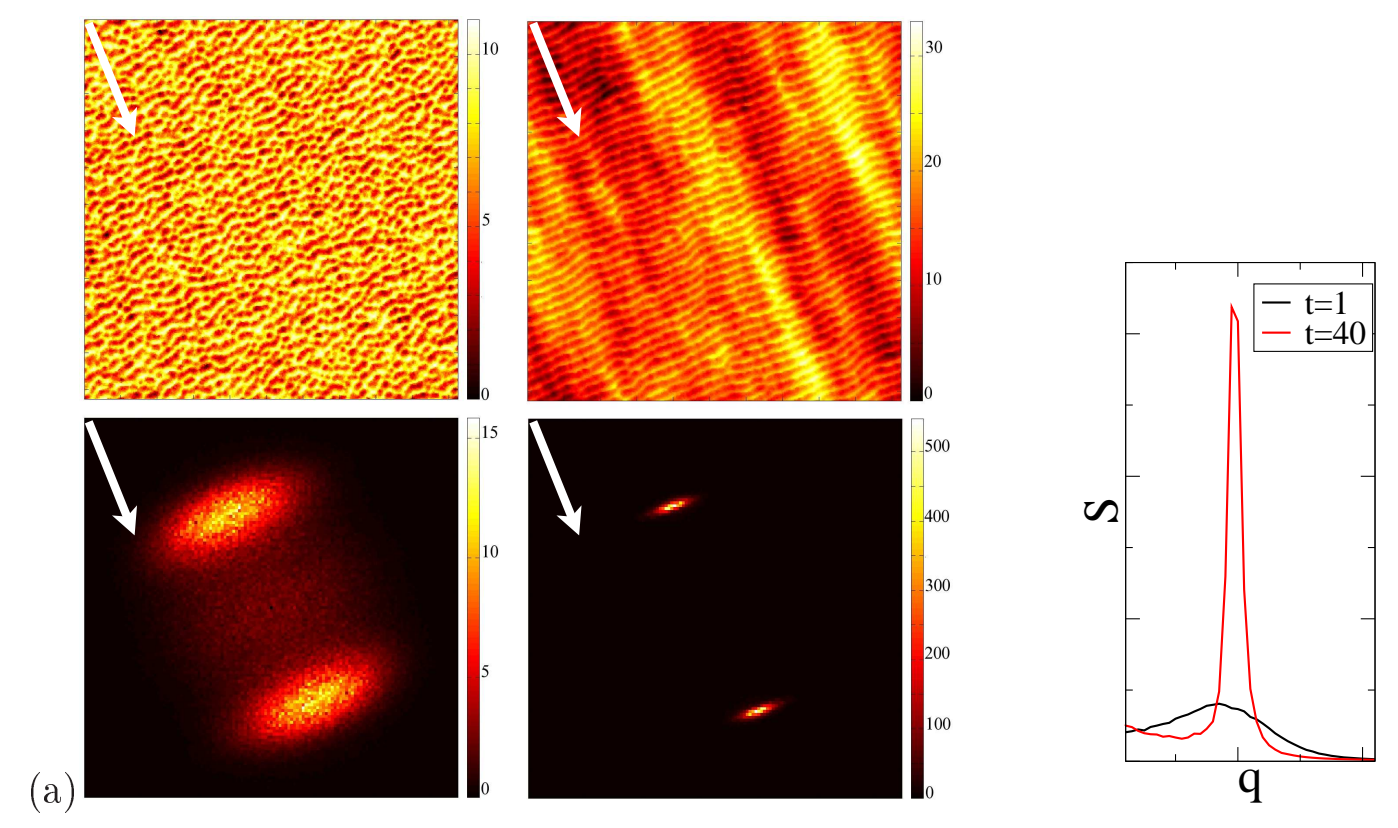

Figure 3.5: (a) upper row: surface profiles with $L=512$ after left: 1 ion/atom, right: 40 ions/atom sputtering at $\theta=50$. lower row: corresponding 2-d Fourier transform of above profiles. (b) power spectral density (averaged over azimuthal angle) of surface profiles shown in (a). By increasing the sputtering time, order of structures increases and the height of the pick in PSD grows as its width decreases.

$$
S(\vec{q}, t)=S(\vec{q}, 0) \exp (-2 R(q) t)
$$

where $S(\vec{q}, 0)$ is the power spectral density of the surface at $t=0$.

PSD can be measured by many optical scattering methods and also by direct Fourier transform of the surface profile. Peaks in the PSD averaged over angles correspond to the selection of specific wavelength. $R$ can be estimated by measuring the growth rate of peaks height. Periodic arrangement of peaks reflects the periodicity of the surface patterns. Moreover, the angular distribution of the Fourier transform is related to the structural anisotropies. Finally, the width of the PSD is a measure of the degree of order, i.e. the quality of regular patterns. Examples of surface profiles, corresponding 2-d Fourier transform and PSD are depicted in Fig. 3.5. 


\section{Chapter 4}

\section{Erosion and diffusion-driven patterns}

Pattern formation in IBS is an outcome of a competition between erosion and lateral diffusion. The kinetic rates of these processes are controlled by ion parameters (e.g. flux and energy) and substrate parameters (e.g. diffusivity and temperature) respectively. In crystalline materials, two different regimes of pattern formation are observed by changing the erosion and diffusion conditions. In a high flux and low temperature regime, erosion events are the dominant processes driving the pattern formation, and diffusion acts only as to smooth the long wavelength fluctuations. Therefore the emerging patterns are ripples oriented in the direction determined by the ion-beam direction, which is compatible with the predictions of the linear BH theory. In a low flux and high temperature regime, diffusion is the dominant factor and therefore patterns beyond the predictions of $\mathrm{BH}$ theory emerge. In this regime, dependent on the diffusion bias of the sample, and the crystallographic orientations, ripples appear which are oriented by crystal anisotropies rather than by the ion-beam direction. Furthermore, other kinds of patterns, e.g. positive or negative pyramids (pits) develop. In the KMC simulations, erosion and diffusion parameters are controlled by changing the rate of ion impacts and diffusion attempt frequency. As a control parameter, we consider $\mathcal{R}=\tau_{i} / \tau_{d}$, where $\tau_{i}$ is the time interval, in which one ion per atom is shot into the surface and $\tau_{d}$ is the waiting time between two diffusion sweeps applied to each atom.

We start this chapter with a brief review of simulation results obtained 
in the erosion-driven regime under varying parameters. Then we introduce models of surface diffusion and discuss the effects induced by combining each of them with erosion events in high flux regime. Finally, we present results covering the crossover from the erosion-driven to the diffusion-driven regime.

\subsection{Erosion}

According to the work of Sigmund as mentioned in section [3.3, the spatial shape of collision cascades as well as the distribution of deposited energy by impinging ions is approximated by a 3-d Gaussian function in almost all the theoretical models (including computer simulations). The distribution function is parametrized by its longitude and latitude expansions $\sigma$ and $\mu$, and is centralized at a point at the distance $a$ (penetration depth) from the point of ion impact on the surface. Using BCA packages (i.e. SRIM (Ziegeler et al. 1985)), it is possible to calculate $\sigma$ and $\mu$ for every combination of ion and substrate type. Systematic studies on the dependence of patterns on these parameters are presented by Yewande et al. (2006). The results can be summarized by a kinetic phase diagram, showing different morphologies (ripples, dots, holes and cellular structures for different values of $\sigma$ and $\mu$.

With Gaussian-shaped collision cascades, pronounced ripples with wavevectors oriented perpendicular to the projection of the ion-beam onto the surface are not obtained in KMC. We demonstrate that this deficiency can be removed by replacing the Gaussian shape by a shape of collision cascades obtained from BCA simulations.

\subsubsection{Dependence of patterns on ion parameters}

By systematic changes in the values of $\sigma$ and $\mu$ for an incidence angle of $\theta=50^{\circ}$, six different types of qualitative behavior corresponding to six different regions in the $(\sigma, \mu)$ space are observed. In Fig. 4.1, these six regions are indicated for $t=3$ ions/atom, at which almost all the surface topographic features are distinct; the corresponding profiles are shown in Fig. 4.2. The boundaries shown in this sketch do not represent abrupt transitions from one topography to another and they change with time.

The following features distinguish the different topographies in Fig. 4.2: 


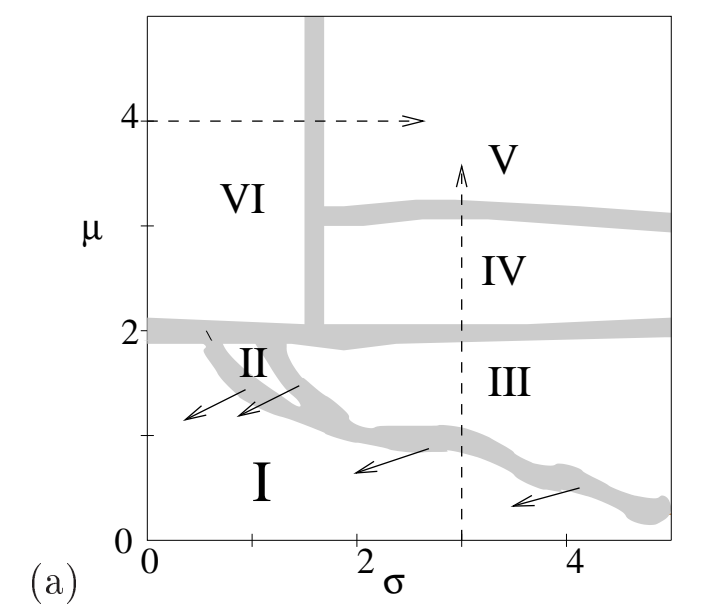

Figure 4.1: Kinetic phase diagram $\left(\theta=50^{\circ}, d=6\right)$. Region I: rough surface; II: holes; III: clear ripples oriented perpendicular to the ion-beam direction; IV: short ripples (resulting from increased $\mu$ ); V: dots; VI: non-oriented structures. The short arrows indicate the evolution of the boundaries between different regions with respect to time. Hence, region III grows at the expense of region I, while region II describes only a short transient.

Region I: rough surface (see Fig. 4.2(I)) which, as time increases, evolves to a hole topography. The "sizes" of the holes grow and finally coalesce to a ripple topography at long times.

Region II: holes are prominent in this region (see Fig. 4.2(II)). Here the "number" of holes increases with time, and again ripples are formed at long times, but at an earlier time than as region I (not shown as separate figure).

Region III: the ripple phase (Hartmann et al. 2002; Yewande et al. 2005). Here ripples form from earliest time. Thus, comparing regions I,II and III, there seem to be two different processes of ripple formation. Ripples can be formed quickly by evolving directly from a slightly rough surface, or can be formed slowly via the creation of holes, which coalesce to ripples on longer time scales. Note that in regions I and II, the resulting ripple wavelength is smaller than the size of the holes generated at smaller time, while in region III the ripple wavelength is larger than the tiny holes.

Region IV: consists of a mixture of dots and short ripples, which eventually give way to the dot "phase" (region V), as $\sigma$ is increased. Hence, this region seems to "interpolate" between regions III and V.

Region V: consists of dots. These dots are formed on some ripple-like struc- 

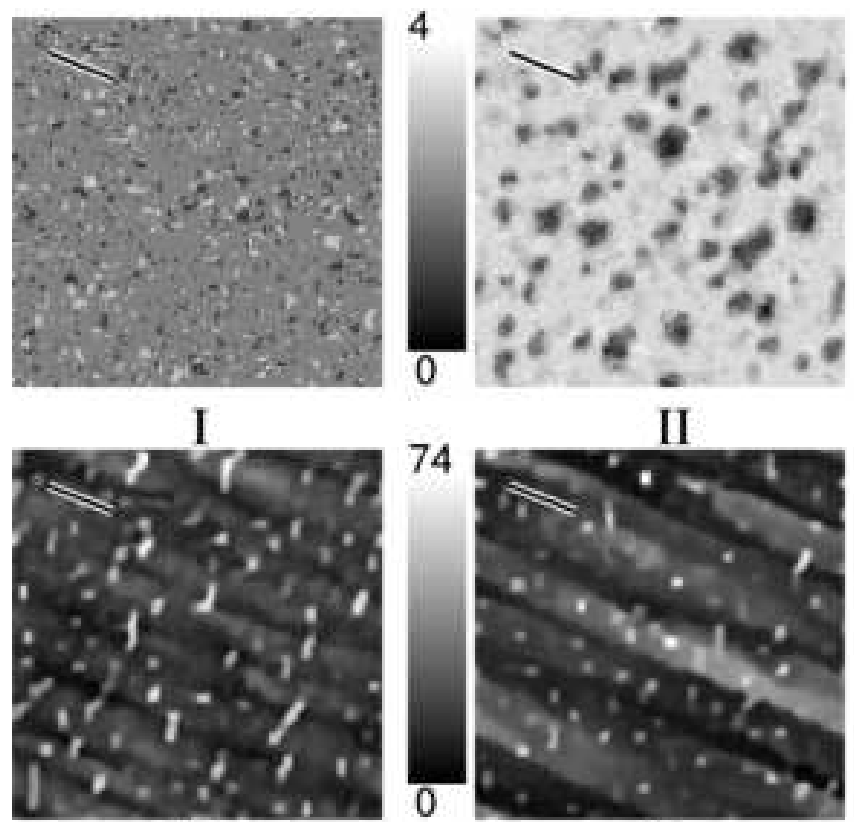

(a)

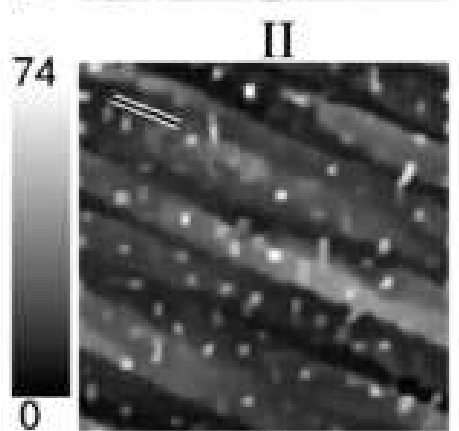

V

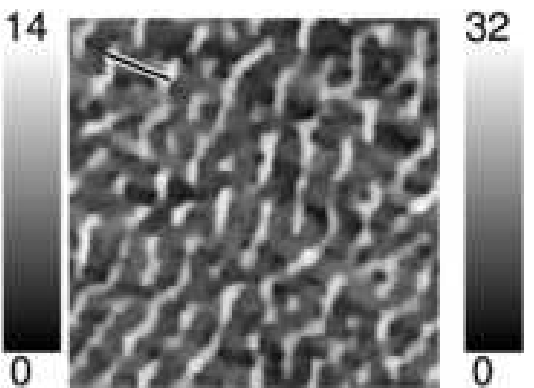

III

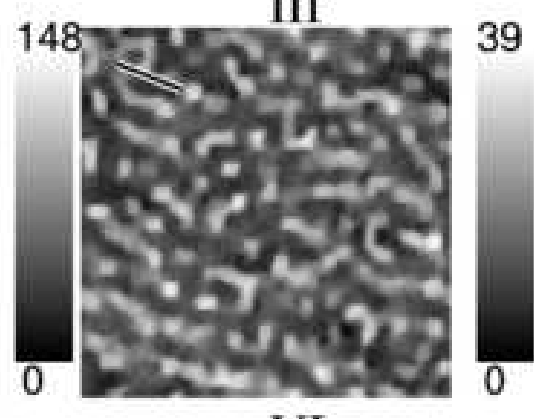

VI

Figure 4.2: Profiles for parameters chosen from each topographic region in Fig. 4.1, $\theta=50^{0}, d=6, t=3.0$. (I) $\sigma=1, \mu=0.5$; (II) $\sigma=1, \mu=1.5$; (III) $\sigma=3, \mu=1.5$; (IV) $\sigma=4, \mu=2.5$; (V) $\sigma=5, \mu=5$; (VI) $\sigma=0.5, \mu=5$. The bar, on all profiles, denotes the ion-beam direction. 
tures oriented parallel to the ion-beam direction.

Region VI: consists of non-oriented structures exhibiting a typical length scale, but only a slight orientation preference parallel to the ion-beam.

\subsubsection{BCA model based erosion}

In KMC simulations beyond incidence angles of $\theta \approx 70^{\circ}$ the ripples with wavevectors parallel to the ion-beam direction vanish. In Fig. 4.3 (c) we see simulation results obtained with a Gaussian shape of the collision cascades. Note that small ripple fragments remain, but the rotated ripples, which are expected from linear BH theory and observed in experiments, do not appear. We have already noticed in section 3.1 that the shape of cascade, which is obtained from BCA simulations, differs significantly from a Gaussian. The right panels of Fig. 4.3 ((b) and (d)) show simulations with this modified shape. Now the rotated ripples observed in experiments appear as pronounced structures.

\subsection{Diffusion}

Although the important role of surface relaxation events in pattern formation by IBS is evident, it is not yet clear what is the most realistic model of atomic diffusion in the discrete SOS framework. There are different proposals (mostly arising from MBE studies), which can partially explain the features observed in experiments, but none of them have the capability to describe atomic mobility in general form. Measuring the growth exponents corresponding to each model and comparing them to the values obtained in MBE experiments is a way to determine the relevant model to each condition of experiments. However, entanglement of diffusion and erosion makes conclusions more difficult in the case of IBS. As mentioned in section 3.3.2, we have implemented different diffusion models in our KMC simulations. In the following, some of well studied diffusion models are introduced briefly. We describe them as discrete particle algorithms as well as continuum equations for the evolution of the height profile $h(\mathbf{x}, t)$. 
(a)

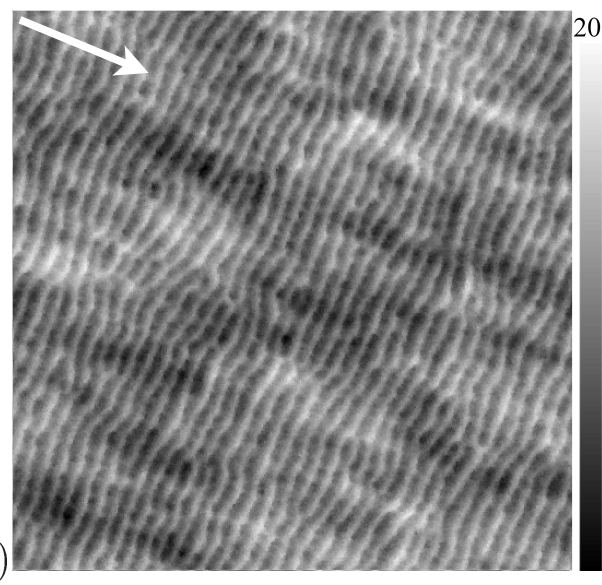

(c)

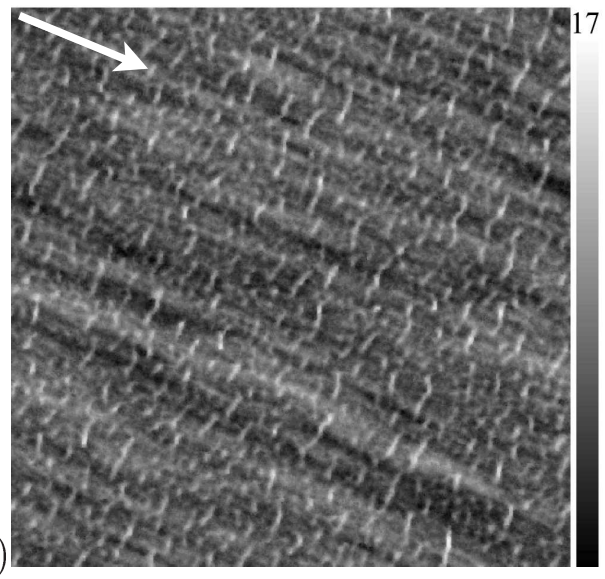

(b)

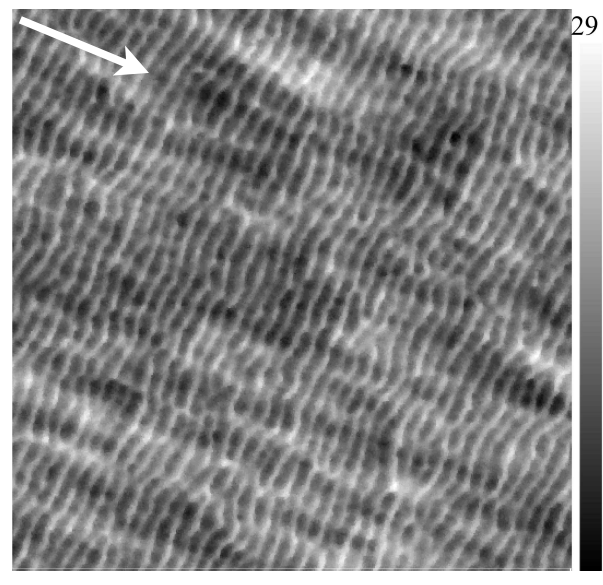

(d)

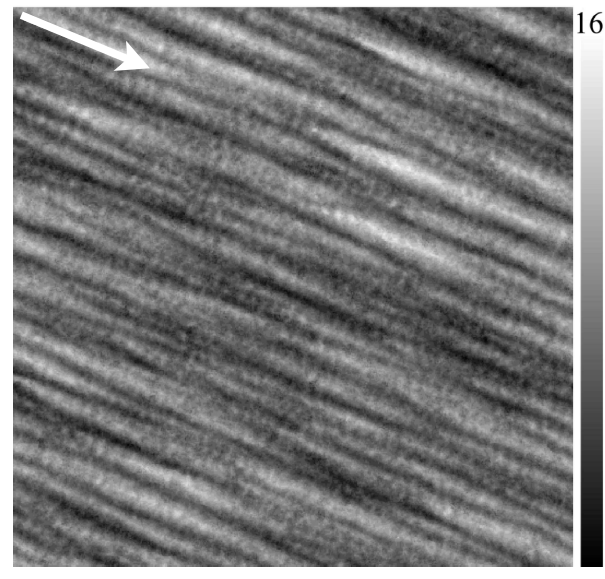

Figure 4.3: Two sets of simulations based on Sigmund's energy distribution and Feix et al. for (a) and (b) $\theta=50^{\circ}$, (c) and (d) $\theta=80^{\circ}$. The left panels correspond to Sigmund's distribution and the right panels to Feix et al. distribution. The evolved patterns are qualitatively the same for $\theta=50^{\circ}$, whereas for $\theta=80^{\circ}$ patterns emerged from Feix et al.'s distribution are more similar to the experimental results (one example is depicted in Fig. 2.10). 


\subsubsection{Irreversible models}

In the irreversible models of diffusion, a particle sticks to a neighboring particle and keeps this position permanently. The irreversible attachments correspond to models for low temperature regimes, where breaking the bonds is highly unlikely due to the large binding energies.

\section{Family model}

Family (1986) has considered effects of surface relaxation in scaling behavior of growing surfaces by the use of discrete models. By adding a very simple relaxation process to the random deposition of particles to a $(1+1)$-d surface, he reached a new universality class. In the Family model, every arriving particle on the surface is allowed to relax to one of the nearest neighboring sites if it has lower height than the initial target site. This process leads to reduction of roughness and smoother growth of surface as compared to random deposition growth. The scaling exponents (defined in section 3.5.1) of the Family model in 2-d are $\alpha=0.48 \pm 0.02$ and $\beta=0.24 \pm 0.01$. Relaxation to farther neighboring sites, for example next nearest neighbors, does not change these scaling exponents. The Family model is shown to belong to Edwards-Wilkinson (EW) universality class.

Studying surface fluctuations in a settled granular material, Edwards and Wilkinson (1982) found the simple continuum equation to describe the process by which a particle settles and comes to rest on the existing surface of the packing. In EW description, the corresponding term in the growth equation of a randomly driven surface is $D \nabla^{2} h$, where $D$ is acting like a surface tension coefficient which smooths the height fluctuations of the surface. If this relaxing term is combined with a random noise of adding particles, the growth equation becomes

$$
\frac{\partial h(\mathbf{x}, t)}{\partial t}=D \nabla^{2} h+\eta(\mathbf{x}, t)
$$

Scaling analysis of EW equation in $\mathrm{d}+1$-dimension, leads to the following values for scaling exponents (Barabási and Stanley 1995)

$$
\alpha=\frac{2-d}{2}, \quad \beta=\frac{2-d}{4}, \quad z=2 \quad \text {. }
$$




\section{Wolf-Villain, Das Sarma-Tamborenea and Lai-Das Sarma models}

The first models for non-equilibrium growth processes at atomistic level including lateral motion driven by the binding energetics were introduced by Wolf and Villain (1990) and Das Sarma and Tamborenea (1991) independently. In both models (WV and DT), a deposited particle relaxes to the neighboring sites if it increases the number of its in-plane neighbors, i.e. particles move to increase the number of their lateral bonds. In WV, there is preference to maximize the number of in-plane bindings, whereas in DT, hops to the sites with larger (not necessarily largest) bindings are performed with equal probability. A more detailed version of DT was introduced by Lai and Das Sarma (1991) (LD), in which the particles landed onto a kink (i.e. in 1-d, the site with one neighbor) are allowed to jump to a neighboring site with a smaller step height. In this process, upward and downward movements are both accepted. Scaling exponents, measured for these models are shown in Table 4.1,

Another discrete model which shows correspondence to $\nabla^{4}$ universality class is the so called "larger curvature model" (LC) introduced by Kim and Das Sarma (1994). In LC, a particle relaxes to one of its nearest neighbors $x$, where the curvature of the surface at this point $h(x+1)+h(x-1)-2 h(x)$ is larger than at the original site. The LC model is shown exactly to belong to $\nabla^{4}$ universality class.

The continuity equation is a starting point to obtain a continuum description for surface diffusion driven by energetics of atomic bindings, which implies that the number of particles remains constant during lateral motion

$$
\frac{\partial h(\mathbf{x}, t)}{\partial t}=-\nabla \cdot \mathbf{j}(\mathbf{x}, t)
$$

The surface current density $\mathbf{j}$ is driven by the gradient of the chemical potential $\mu(\mathbf{x}, t)$, i.e.

$$
\mathbf{j}(\mathbf{x}, t) \propto-\nabla \mu(\mathbf{x}, t)
$$

Since the origin of chemical activated transportation are binding energies of neighboring atoms, and the number of neighbors of an atom located at a point is proportional to the curvature of surface profile at that point, we find that the chemical potential in such system is

$$
\mu(\mathbf{x}, t) \propto-\nabla^{2} h(\mathbf{x}, t) .
$$


Combining Eq. 4.3, 4.4 and 4.5, the continuum expression describing surface diffusion reads like

$$
\frac{\partial h(\mathbf{x}, t)}{\partial t}=-K \nabla^{4} h
$$

where $K$ is a diffusion rate (which in the case of thermally activated diffusion scales with $\left.1 / k_{\mathrm{B}} T\right)$. This corresponds to the continuum description of LC. If the diffusion process is combined with a random deposition of particles, the growth equation of the surface reads

$$
\frac{\partial h(\mathbf{x}, t)}{\partial t}=-K \nabla^{4} h+\eta(\mathbf{x}, t)
$$

which has the scaling exponents (Barabási and Stanley 1995)

$$
\alpha=\frac{4-d}{2}, \quad \beta=\frac{4-d}{8}, \quad z=4 .
$$

\section{Non-linear diffusive terms}

The most relevant term in the sense of scaling behavior, which can be written as a gradient of a chemical potential, was introduced by Lai and Das Sarma (1991). It is given by $\nabla^{2}(\nabla h)^{2}$ and corresponds to continuum description of LD. And when combined with Eq. 4.7, we get

$$
\frac{\partial h(\mathbf{x}, t)}{\partial t}=-K \nabla^{4} h+\lambda_{1} \nabla^{2}(\nabla h)^{2}+\eta(\mathbf{x}, t)
$$

where $\lambda_{1}$ is another diffusion rate. Scaling exponents calculated for the mentioned growth equation are (Lai and Das Sarma 1991)

$$
\alpha=\frac{4-d}{3}, \quad \beta=\frac{4-d}{8+d}, \quad z=\frac{8+d}{3} .
$$

Many numerical works are done to determine the scaling exponents of the mentioned discrete models. A summary of the most well-known calculated values for 2-d systems is given in Table 4.1,

\subsubsection{Hamiltonian models}

In another class of diffusion models called "Hamiltonian models", atomic hops are associated with a change in a Hamiltonian $\mathcal{H}$ and acceptance of a hop is allowed according to a Metropolis criterion (Siegert and Plischke 1992; Krug et al. 1993; Siegert and Plischke 1994). In this model, all the particles at the top-most 
Table 4.1: A summary of calculated values of the growth $\beta$ and roughness $\alpha$ exponents for different irreversible discrete diffusion models in $(1+1)$-d.

\begin{tabular}{|c|c|c|c|}
\hline & $\alpha$ & $\beta$ & Ref. \\
\hline Family & $0.48 \pm 0.02$ & $0.24 \pm 0.01$ & (Family 1986) \\
WV & $1.4 \pm 0.1$ & $0.36 \pm 0.072$ & (Wolf and Villain 1990) \\
DT & $1.47 \pm 0.10$ & $0.375 \pm 0.005$ & (Das Sarma and Tamborenea 1991) \\
LD & $1.05 \pm 0.10$ & $0.340 \pm 0.015$ & (Lai and Das Sarma 1991) \\
LC & $1.5 \pm 0.1$ & $0.375 \pm 0.010$ & (Kim and Das Sarma 1994) \\
\hline
\end{tabular}

layer are the subject of diffusion. The diffusing particle hops from site $i$ to a neighboring site $j$ with the normalized rate

$$
w_{i \rightarrow j}=\left[1+\exp \left(\frac{1}{k_{\mathrm{B}} T} \Delta \mathcal{H}_{i \rightarrow j}\right)\right]^{-1}
$$

where $\Delta \mathcal{H}_{i \rightarrow j}$ is the change in Hamiltonian due to the hop from site $i$ to site $j$. The Hamiltonian has the general form of an unrestricted SOS model

$$
\mathcal{H}=\frac{1}{2} J \sum_{\langle i, j\rangle}\left|h_{i}-h_{j}\right|^{n}
$$

where $\langle i, j\rangle$ counts for all the nearest neighbors, $J$ is coupling constant and $n$ is a positive integer number. The Hamiltonian model is able to reproduce some significant features of atomic transport mechanisms in a wide range of materials. For example, there is no energy cost for diffusion of a single adatom or vacancy on a flat surface in the described model which leads to the same diffusivity for adatoms and vacancies on (001) facets. This feature is extensively reported for metallic surfaces (Chan and Chason 2007). The model is also able to simulate the so-called "Schwoebel effects", which refers to the repulsion of diffusing atoms from descending step edges and an observed up-hill current on stepped surfaces (for more details see section 4.2.4). Siegert and Plischke (1994) showed that for $n=1,2$ and $n>2$, the mentioned model induced negative, zero and positive Schwoebel effects respectively and in general, increasing $n$ leads to stronger Schwoebel effects.

Another version of Hamiltonian models for diffusion is introduced by Stepanova et al. (2005), in which the associated Hamiltonian is

$$
\mathcal{H}=\frac{1}{2} a \gamma\left(\left|\kappa_{i}+\kappa_{j}\right|\right)
$$


where $\kappa$ is the local curvature, $a$ is the inter-atomic distance and $\gamma$ is the energy of one atomic bond. The mentioned Hamiltonian simulates the tendency of atoms to diffuse to the positions with more neighbors.

\subsubsection{Thermally activated models}

Another successful discrete model for surface diffusion is known as "thermally activated diffusion" models (for example see Śmilauer et al. (1993)), and is based on an Arrhenius form of hopping rate

$$
k(E, T)=k_{0} \exp \left(\frac{-E}{k_{\mathrm{B}} T}\right)
$$

where $k_{0}=2 k_{\mathrm{B}} T / h_{p}$ is attempt frequency of a surface adatom $\left(h_{p}\right.$ is Planck constant). The energy barrier $E$ is the average of the transition state of the corresponding hop. In KMC it is expressed by bond energies of the diffusing adatom. There are different variants of expressing $\mathrm{E}$ by bond energies used in the literature, which we now introduce.

\section{Bond-breaking models}

In these models, the energy barrier $E$ consist of the energy of all bonds of the moving atom at its initial position. As the energetics of in-plane bonds $\left(E_{\mathrm{nn}}\right)$ is different from the one between the atom and the substrate $\left(E_{\mathrm{s}}\right)$, the total binding energy is

$$
E=E_{\mathrm{s}}+n E_{\mathrm{nn}}
$$

where $n$ is the number of in-plane nearest neighbors. Some authors also take contributions from the next-nearest-neighbor bonds into account (Johnson et al. 1994; Tok et al. 2004). An extra Schwoebel barrier may also be added to the binding energies (for more detail see section 4.2.4).

Note that in most materials, energy barriers are not known precisely, and especially in crystalline substrates, barriers against movements in different directions are different. The main drawback of the bond-breaking models is that they generally imply some features of atomic motions, which are not in accordance with experiments or molecular dynamics simulations. For example it is frequently observed that adatoms captured by a step-edge display fast 
diffusion along the step, but detachment from the step occurs at a much larger rate although the bond-breaking model would predict equal rates.

Generally, all features, which are due to the fact that the transition state energy can not entirely be expressed by the initial state of the hop, can not be also described by bond-breaking models.

\section{Net-bond-breaking (Kawasaki-type) models}

A better and more realistic representation of $E$ makes use of both the initial and the final state of a hop. For example one could assume that $E$ is composed of a constant term $E_{\mathrm{s}}$ and an extra activation energy $\Delta E=\max \left[0,\left(\mathrm{n}_{\mathrm{i}}-\mathrm{n}_{\mathrm{f}}\right) \mathrm{E}_{\mathrm{nn}}\right]$ given by the binding energy difference of initial $(i)$ and final $(f)$ states (or zero, if the difference is negative, i.e. if binding energy is gained by the hop).

Note that now barriers to diffusion of an adatom and a vacancy on a flat surface are equal and there is no contribution from in-plane bonds to the barrier against diffusion along an step-edge.

\section{Attempt rate, absorption of substrate term}

In our simulations we use $E_{\mathrm{s}}=0.75 \mathrm{eV}$ and $E_{\mathrm{nn}}=0.18 \mathrm{eV}$. These values are within plausible bounds, but they do not aim at modeling a specific material. Note that in a KMC simulation by these values, most of the attempts would be rejected for common experiment temperatures $(0.04 \cdots 0.1 \mathrm{eV})$. However, the attempt frequency is rather high; for example $k_{0}$ at room temperature is $\sim 10^{13} \mathrm{~s}^{-1}$. Performing so many attempts for each atom is absolutely out of the power of the available machines. So an alternative to speed up the simulations is to rescale the attempt frequency $k_{0}$ by a factor of $\exp \left(-E_{\mathrm{s}} / k_{\mathrm{B}} T\right)$. The rescaled attempt frequency $k_{1}=2 k_{\mathrm{B}} T / h_{p} \exp \left(-E_{\mathrm{s}} / k_{\mathrm{B}} T\right)$ is strongly temperature-dependent. In Table. 4.2, values calculated for some given temperatures and $E_{\mathrm{s}}=0.75 \mathrm{eV}$ are reported. Note that a slight increase of $0.05 \mathrm{eV}$ in the value of $E_{\mathrm{s}}$ (an example used by Chason et al. (2006)) leads to a reduction of one order of magnitude in the value of $k_{1}$. In the current work, rescaled attempt frequency of $750 \mathrm{~s}^{-1}$ is used as a default value, but a systematic investigation on the role of attempt frequency in pattern formation in IBS is presented in section 4.3.2. 
Table 4.2: Attempt frequency of atomic hops for the Arrhenius model of diffusion; for the fixed value of substrate binding energy $E_{\mathrm{s}}=0.75 \mathrm{eV}$ and different substrate temperatures. By slight changes of temperatures, the attempt frequency changes some orders of magnitude.

\begin{tabular}{|c|c|c|c|c|c|c|}
\hline$T(\mathrm{~K})$ & 300 & 350 & 400 & 500 & 600 & 700 \\
\hline$k_{1}\left(\mathrm{~s}^{-1}\right)$ & 3 & 230 & $5.9 \cdot 10^{3}$ & $5.7 \cdot 10^{5}$ & $1.2 \cdot 10^{7}$ & $1.2 \cdot 10^{8}$ \\
\hline
\end{tabular}

\section{Temperature}

In addition to the mentioned effects on hopping attempt frequency induced by slight changes of temperature, probability of acceptance of different transport events, like detachments and inter-layer hops are also temperature dependent. To evaluate the role of temperature in the growth process of a surface, Tamborenea and Das Sarma (1993) performed simulations on $1+1$ dimensional MBE with $E_{\mathrm{s}}=1 \mathrm{eV}$ and $E_{\mathrm{nn}}=0.3 \mathrm{eV}$ for different temperatures and also different ratios of deposition and diffusion rates. They observed three main regimes in temperature: (i) low temperature regime, where the deposited particles are frozen, and no effective diffusion occurs (growth exponents similar to ones for random deposition), (ii) an intermediate temperature regime, with an effective diffusion with growth exponents similar to what is predicted by linear theory in the form of $\nabla^{4}$, and (iii) a high temperature regime, in which diffusion smooths the surface and the observed scaling is similar to that of EW. Similarly, three regimes has been defined in deposition experiments, based upon the ratio of diffusion and deposition rate. Increasing the diffusion rate or decreasing the deposition rate is equivalent to an increase in temperature.

\subsubsection{Ehrlich-Schwoebel effects, pattern formation by dif- fusion}

Diffusion does not always flatten the surface, there are some kinds of thermally activated transport mechanisms which also induce instabilities. This so-called Ehrlich-Schwoebel (ES) effect is the outcome of an effective repulsion from 
descending step edges. If an adatom diffusing on a terrace reaches an ascending step, it is most probable that it will stick to the step-edge and less likely to detach again. In contrast, there is a barrier which repulses the adatom if the adatom approaches a descending step. The repulsion induces an uphill current which increases the slope of underlying structures.

Although there is no first principle derivation of the current density $\mathbf{j}_{\mathrm{ES}}$ of an ES current, there are two phenomenological models which are used frequently, the first gives (Johnson et al. 1994)

$$
\mathbf{j}(\nabla h) \propto\left(\frac{\ell_{d}^{2} \nabla h}{1+\left(\ell_{d} \nabla h\right)^{2}}\right)
$$

and the second one (Krug 1995)

$$
\mathbf{j}(\nabla h) \propto(\nabla h)\left(1-\zeta(\nabla h)^{2}\right) .
$$

$\ell_{d}$ and $\zeta$ are calibrating parameters. Note that the second form implies a reversal of the current direction (from uphill to downhill), which is in accordance whit experiments.

To implement this effect in discrete models of diffusion, one may either set $n>2$ in Hamiltonian models or add an extra barrier to hopping barriers in thermally activated diffusion models as the following.

$$
E_{\mathrm{ES}}(i \rightarrow f)= \begin{cases}E_{\mathrm{ES}} & \text { if } \mathrm{f} \text { is in plane with } \mathrm{i} \text { and } \\ 0 & \text { at the upper edge of a step } \\ \text { otherwise }\end{cases}
$$

In this present work the Schwoebel barrier is set to $E_{\mathrm{SB}}=0.15 \mathrm{eV}$. A schematic picture of the ES effect is shown in Fig. 4.4 and a typical morphology induced by ES effects in MBE depicted in Fig. 4.5,

\subsubsection{Diffusion in compound systems, clustering}

To assess the features of diffusion and clustering of a sub-mono-layer coverage of (metallic) adatoms on flat and pre-rippled surfaces, we have performed KMC simulations. These simulations will later be extended to include ion-beam erosion (in chapter 5), but here, we first focus on the patterns induced purely by diffusion of $\mathrm{Ag}$ atoms with constant coverage of $30 \%$ of a mono-layer on 
a)

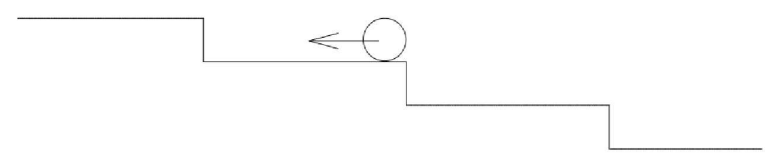

$\mathrm{E}$

b)

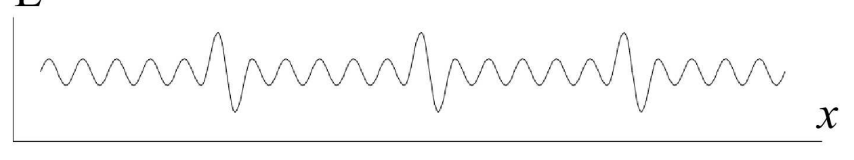

Figure 4.4: Schematic picture of the ES effect: a) a diffusing adatom on a vicinal surface is repelled from a step edge; b) corresponding lattice potential showing the ES barriers at step edges. Adapted from Siegert (1995).

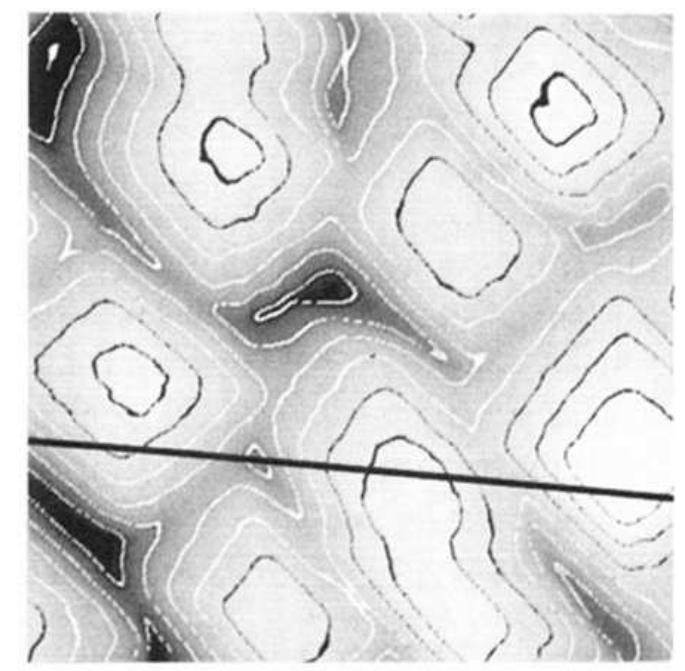

Figure 4.5: Top-view image of $65 \times 65 \mathrm{~nm}^{2} \mathrm{Fe}(001)$ sample showing the pyramid-like surface structures after epitaxial growth. Adapted from Thürmer et al. (1995). 
a Si surface. The corresponding parameters for the diffusion of the metallic atoms are given by $E_{\mathrm{s}}=0.48 \mathrm{eV}$ and $E_{\mathrm{nn}}=0.36 \mathrm{eV}$ (no ES barrier). These parameters are taken from ab initio simulations for Ag atoms on Si substrate (Kong et al. 2003). In the simulation model, we kinetically forbid all moves, which lead to atoms on top of Ag atoms (Ag atoms stay always in the topmost layer and the height of $\mathrm{Ag}$ layer is restricted to 1 atom)

\section{Metallic clusters on flat templates}

Simulations show that initially, randomly distributed metallic particles at intermediate and high temperatures form clusters which coarsen in time. To quantify the clustering, we consider a 2-d array with the same area as the surface. We flag every site of this array by "A" and "B" if the topmost atom in the corresponding location in the surface profile is a surfactant or substrate atom respectively. Now we count the number of neighboring pairs, e.g. $N_{A-B}$ is the number of $A-B$ pairs. Now we define the clustering coefficient $C$ as

$$
C=\left(\frac{N_{A-A}+N_{B-B}}{N_{A-B}}\right)\left(\frac{2 c_{A} c_{B}}{c_{A}^{2}+c_{B}^{2}}\right)
$$

where $c_{B}$ is the surface coverage of surfactants and $c_{A}=1-c_{B}$. Note that, for random distribution of particles $N_{\alpha-\beta} \propto c_{\alpha} c_{\beta}$ and therefore $C=1$, whereas for very large system size, complete segregation of particles corresponds to $C=\infty$ (because $N_{A-B}$ increases linearly with the system size, while $N_{A-A}$ and $N_{B-B}$ increase quadratic). In Fig. 4.6, $C$ as a function of temperature for different simulation time is shown. For very low temperatures, no clustering occurs even for long-time runs. In intermediate temperature, small size clusters form and grow in time slowly, whereas in high temperature regime an strong clustering is observed. The diffusivity of particles is increased by increasing the temperature and in the fixed simulation duration, the particles have more chance to meet each other and since their binding energy is rather high, as they attach, it is very unlikely to detach again.

\section{Metallic clusters on pre-sputtered templates}

The same simulations are now performed using a sinusoidally modulated substrate template. Examples of distribution of particles at different run times and temperatures are depicted in Fig. 4.7. Note the following features: 


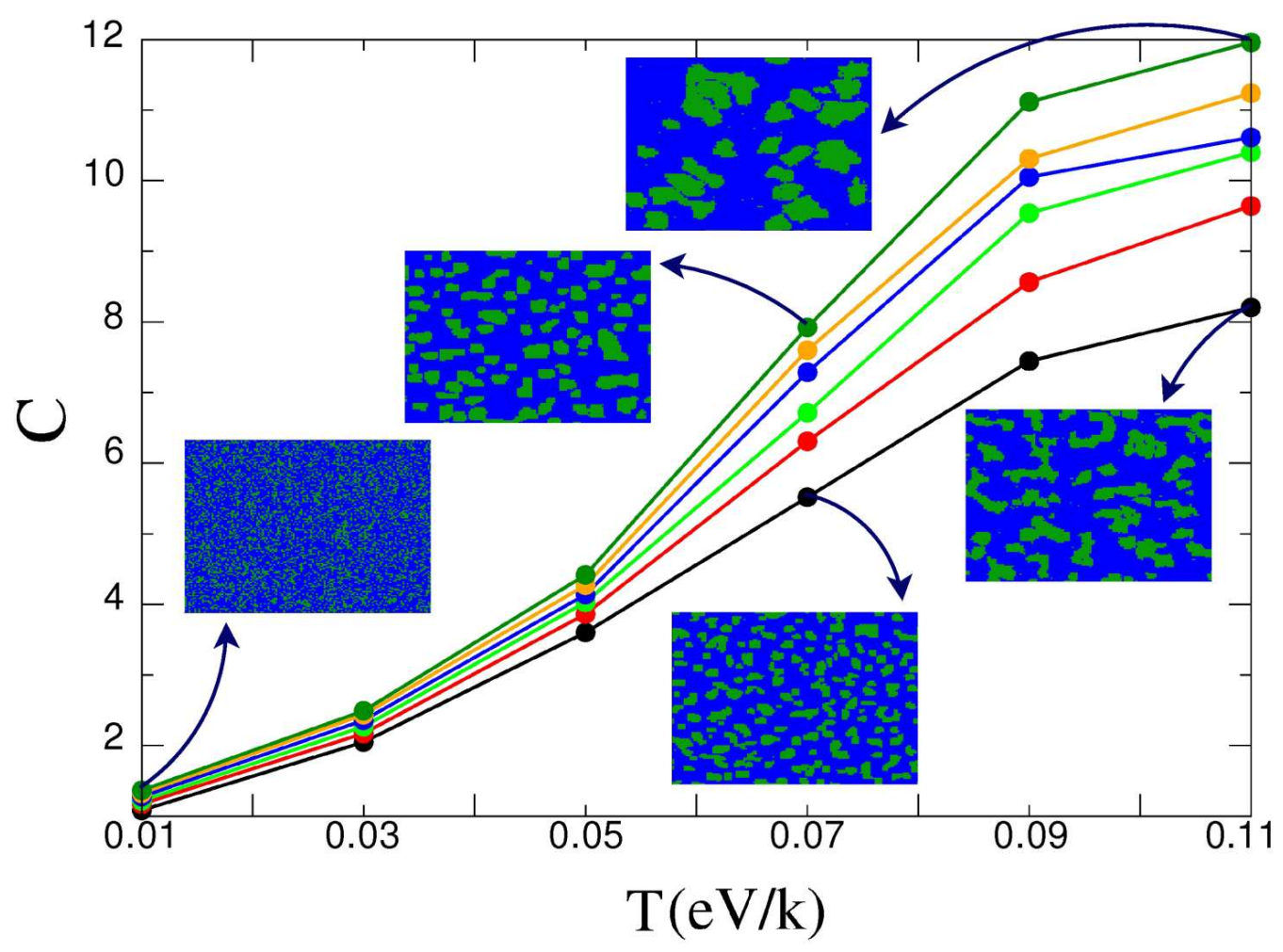

Figure 4.6: Clustering evolution of particles on a flat template as a function of temperature. Different colors correspond to different simulation times. By increasing the temperature, particles make larger clusters. In all cases the size of clusters tends to increase, although the increase in low temperature is very slight. For the definition of $c$ see the main text. 


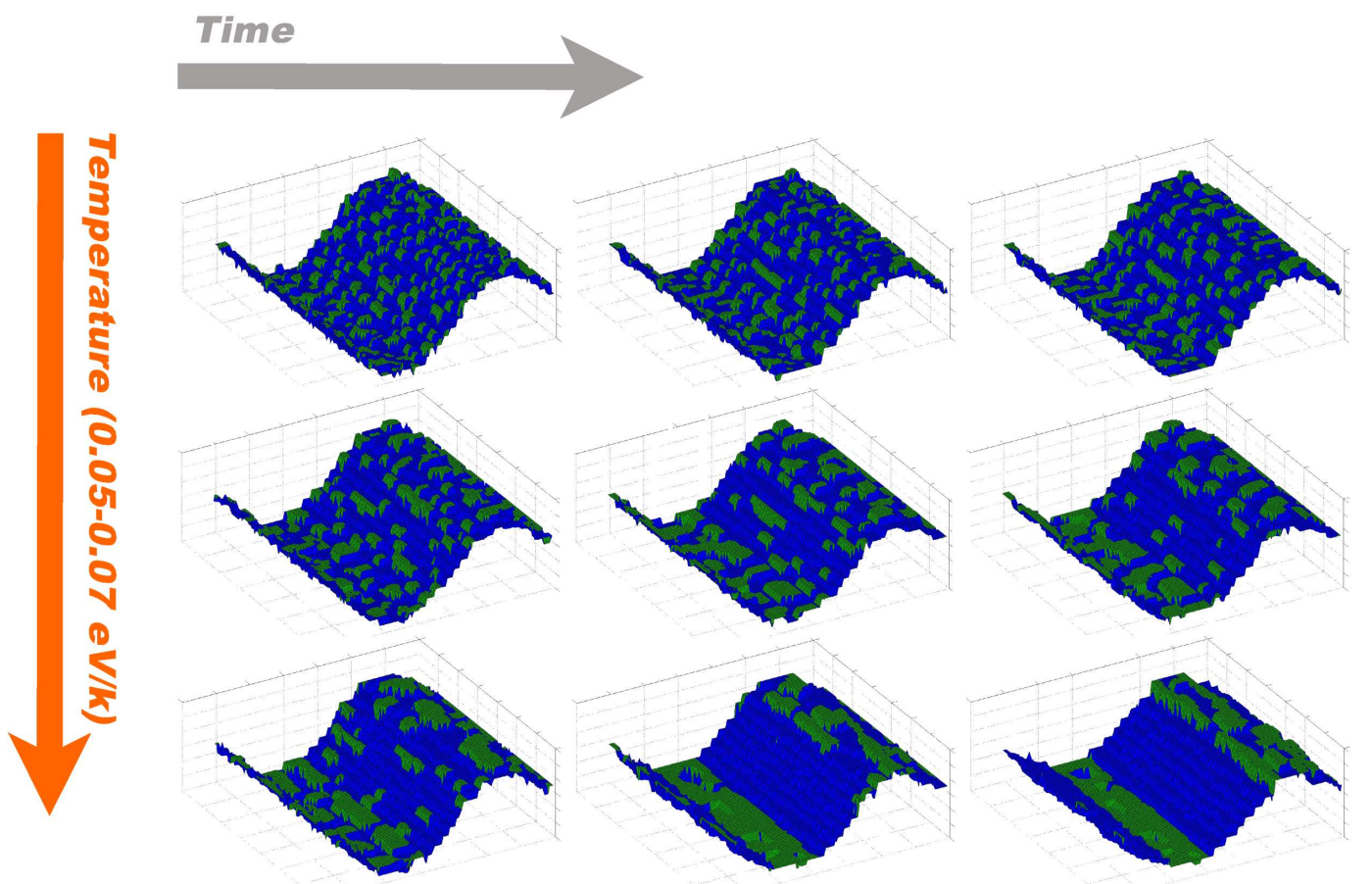

Figure 4.7: Clustering of particles on a sinusoidal template depending on temperature and time. By increasing the temperature, particles make larger clusters. In all cases the size of clusters tends to increase, although the increase in low temperature is very slight. Large clusters formed in high temperature, located mainly in the valleys and plateaus, although there is a preference for valleys in compare to the plateaus.

(i) Particles accumulate in 1-d arrays ("nano-wires") parallel to the alignment of template ripples;

(ii) The width of nano-wires reaches a maximum size proportional to the wavelength of template ripples at enough time scales

(iii) Nanowires have the tendency to form at valleys of templates, the next preferred locations are the hilltop ridges.

All three features have been found in experiments ( $\mathrm{Ag}$ on $\mathrm{Si}$ ) by Oates et al. (2007).

\subsection{Erosion-Diffusion interplay}

In this section we focus on the study of morphologies emerging in KMC simulations of IBS with different diffusion mechanisms. 


\subsubsection{Dependence of patterns on diffusion in the erosive regime}

Diffusion is often considered as an unspecific smoothing mechanism in the erosive regime of IBS experiments on amorphous surfaces. In the continuum theory, it is subsumed as a single term $\sim-B \nabla^{4} h$. In this subsection we show that - contrary to this common belief - different diffusion mechanisms, which would all end up in a $-B \nabla^{4} h$ term in the continuum limit do have profound effects on the morphology, especially in the limit of long times, i.e. high fluences. Figs. 4.8 and 4.9 compare the time evolution of sputtered surfaces under identical conditions of erosion but with different diffusion models. Fig. 4.8 depicts the evolution of surface morphology with a Hamiltonian diffusion model $(n=2, T=0.2 J)$ and Fig. 4.9] shows corresponding results for a net-bondbreaking model. The short-time behavior of both models is very similar, an initial roughening is followed by the formation of ripples. The differences between the two models arise after $\sim 10$ ions/atom of sputtering. At that time, ripples produced in the case of the net-bond-breaking model saturate in amplitude and align more regularly, and the number of defects reduces as time proceeds, whereas the ripples produced by the Hamiltonian model start to get blurred and shorten in length.

In Fig. 4.10 we compare the morphologies of the four main types of diffusion models we have introduced (Wolf-Villain, Hamiltonian, bond-braking and netbond-braking) at very long times.

The irreversible Wolf-villain model (similar to a $T=0$ surface relaxation) produces an extremely ordered stable pattern of straight ripples (even at $t \sim 10^{4}$ ions/atom), whereas the patterns of the Hamiltonian and bondbreaking models loose long-ranged ripple order after a few hundred eroded monolayers. But note that the net-bond-breaking model shows a defect-free ripple pattern after 400 ions/atom. Although the overall diffusivity of the net-bond-breaking is approximately equal to that of the bond-breaking and the Hamiltonian models, net-bond-breaking implies comparable diffusivity of adatoms and vacancies (unlike the bond-breaking model) and is more sensitive to the energetics of bonds than to surface morphology, which dominates the Hamiltonian model. 

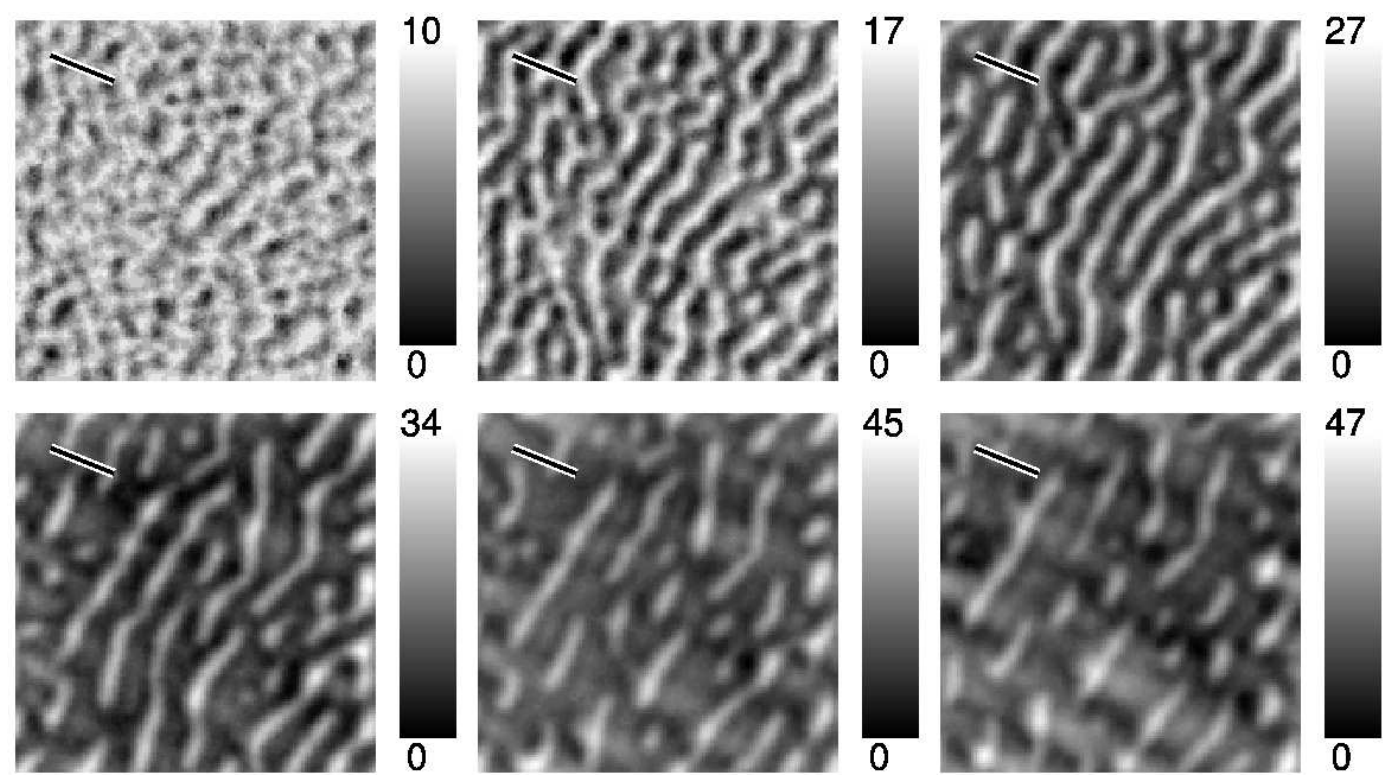

Figure 4.8: Surface profile corresponding to Hamiltonian model of diffusion with $n=2$ and substrate temperature $T=0.2 \mathrm{Jk}_{\mathrm{B}}^{-1}$ and default values of the beam parameters $\left(\theta=50^{\circ}\right)$. Starting from top to bottom and left to right, $t=0.5,1.5,4.0,9.0,14.0$ and 22.0 ions/atom. The ion-beam direction is indicated by the bar. Lateral size of the system $L=256$. After initial formation of ripples, they stabilize and then start to get blurred. 

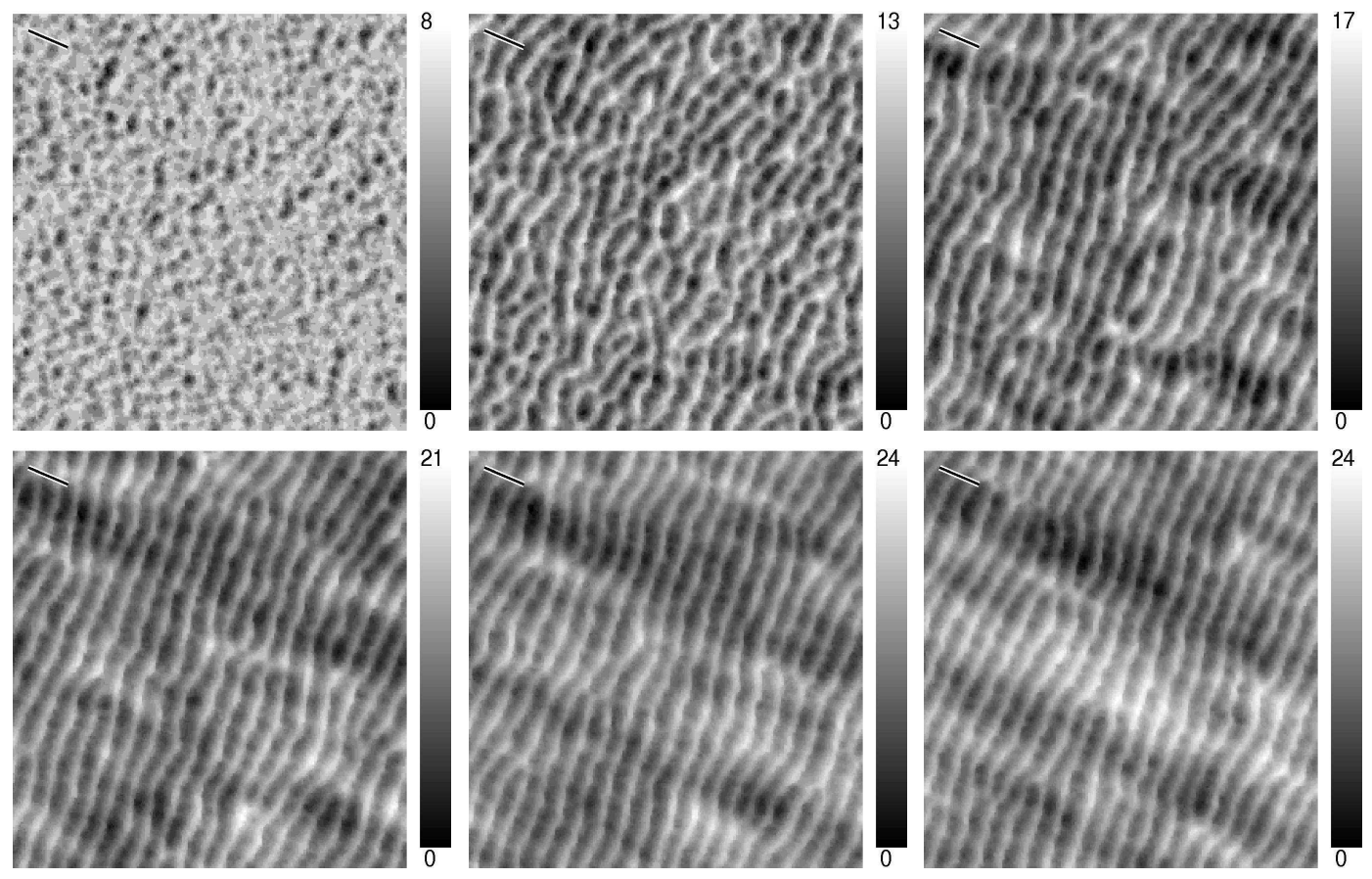

Figure 4.9: Surface profile corresponding to Arrhenius (net-bond-breaking) model of diffusion with substrate temperature $T=600 \mathrm{~K}$, and default values of the beam parameters $\left(\theta=50^{\circ}\right)$. Starting from top to bottom and left to right, $t=0.5,1.5,4.0,8.0,12.0$ and 18.0 ions/atom. The ion-beam direction is indicated by the bar. Lateral size of the system $L=256$. Ripples form after $\sim 3$ ions/atom and grow in lateral size and get more ordered with time. The ripples amplitude saturates for longer times. 


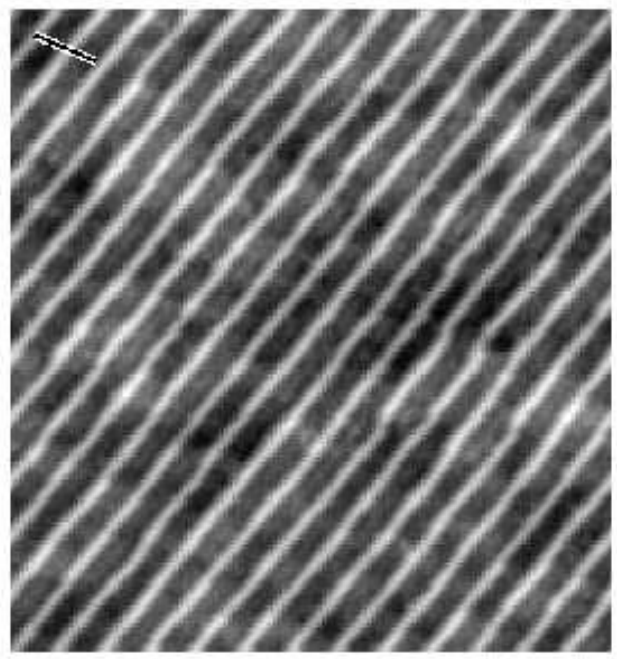

(a)

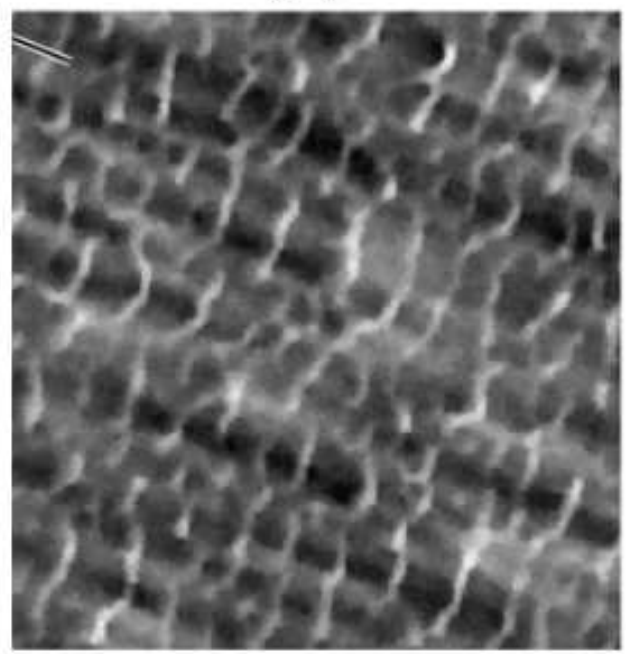

(c)
25

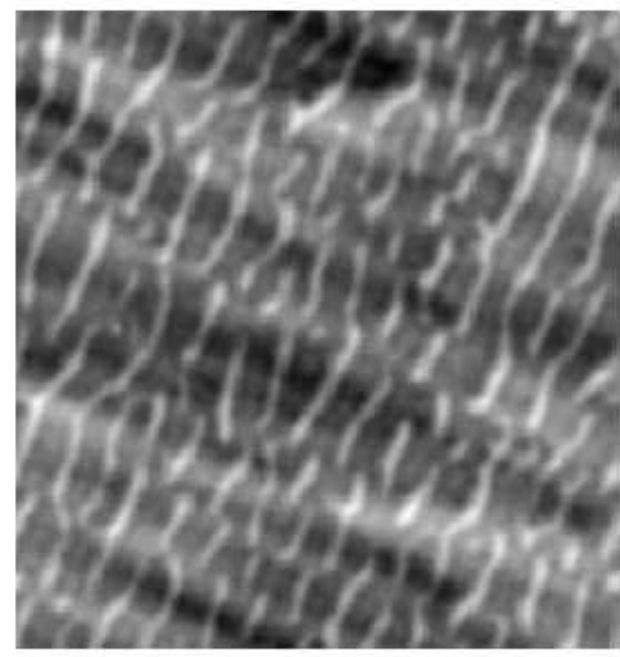

(b)

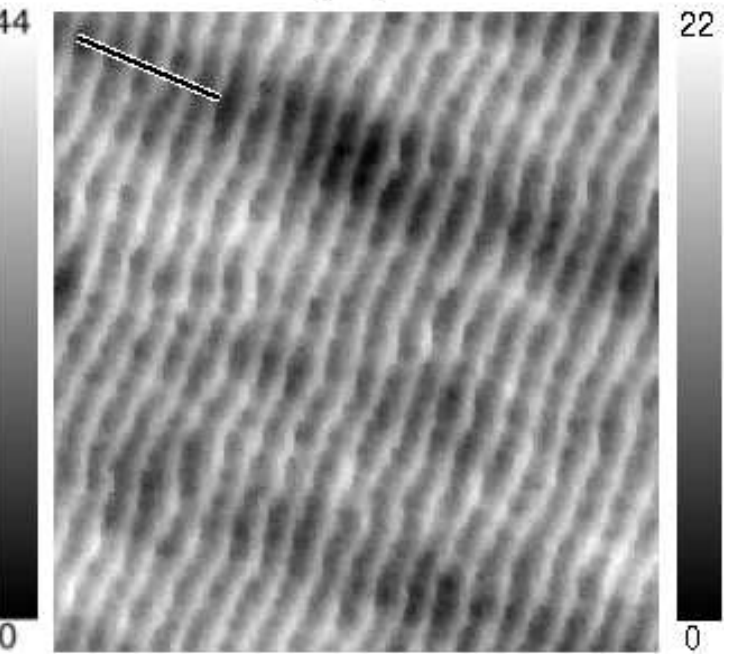

(d)

Figure 4.10: Long time morphologies emerge from different diffusion models (a) WolfVillain for $t \approx 10^{4}$ ions/atom, extremely ordered patterns with tilted orientation in respect to the ion-beam orientation, (b) Hamiltonian with $n=2$, for $t=300$ ions/atom, rational ordered ripples which blur in time gradually, (c) Arrhenius bond-breaking for $t=20$ ions/atom, rather short stable ripples with weak ordering and (d) Arrhenius net-bondbreaking for $t=400$ ions/atom, very ordered ripples whit annihilation of defects by time. 


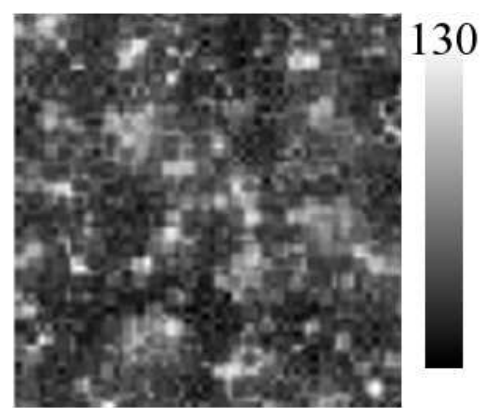

(a)

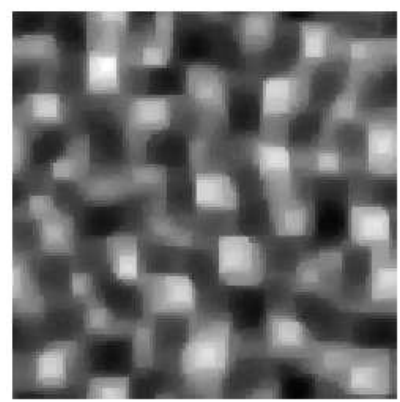

(c)

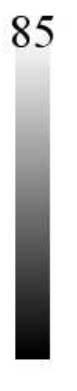

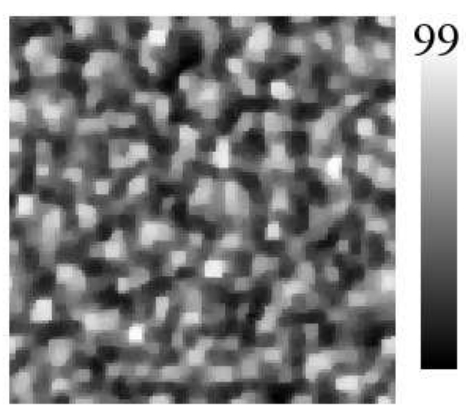

(b)

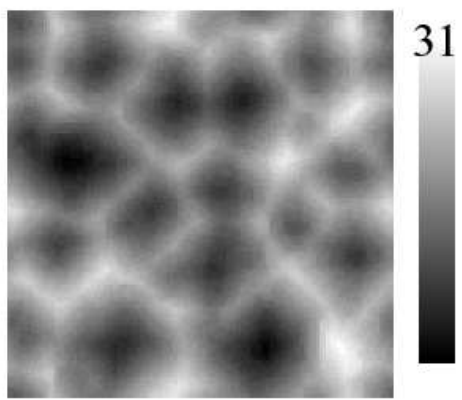

(d)

Figure 4.11: Morphologies $(L=64)$ emerging from different diffusion conditions for $\theta=0^{\circ}$ at $t=10$ ions/atom. (a) no diffusion, (b) Arrhenius bond-breaking diffusion with default parameters, (c) Arrhenius net-bond-breaking diffusion with default parameters and (d) Arrhenius net-bond breaking diffusion with the enhanced attempt rate (see the main text). 


\subsubsection{Crossover from erosive regime to diffusive regime}

We now turn to a discussion of the accessibility of the crossover between the erosive and the diffusive regime in KMC simulations. To estimate the onset of the crossover we adopt an argument from Villain and Pimpinelli (1994) about adatom island formation and stability in MBE, i.e. we simply assume that erosion during IBS is equivalent to a "deposition of vacancies" at a characteristic rate $F$, which is the ion flux times the sputtering yield. The typical length scale of surface structures emerging from diffusion and deposition is given by Villain and Pimpinelli (1994)

$$
\ell_{d} \sim\left(\frac{D}{F}\right)^{1 / 6}
$$

where $D$ is the diffusion coefficient, which in Arrhenius models is $\sim k_{1} a^{2}$. Requiring $\ell_{d}$ to be of the order of typical ripple wavelength produced by IBS ( $10 \mathrm{a}$ in simulations), it implies $k_{1} \sim 10^{6} \mathrm{~s}^{-1}$, which is a factor of $10^{3}$ higher than the default value we use. Enhancing the number of diffusion steps in between two ions soon becomes a computational bottleneck of KMC simulations.

To check if this enhanced rate is sufficient to produce fingerprints of Schwoebel effects, we performed simulation composed of randomly adding and/or removing particles from an initially flat surface. Fig 4.12 shows that pyramid-like structures emerged from this mentioned scenario. Finally, we performed IBS simulations using the enhanced diffusion rate for normal and oblique incidence. Fig. 4.11(b) and (c) show the patterns emerging from normal incidence irradiation with bond-breaking and net-bond-breaking diffusion model. In Fig. 4.11 (d) the diffusion rate of the net-bond-breaking model is enhanced by a factor of $10^{3}$. Only in this case, pyramid structures with edges oriented along $<100>$ directions - hallmark of ES current-induced structures (see Fig. 4.12) - are created. Under oblique incidence, the ripple orientation deviates from the direction of the ion-beam projection into the surface and larger parts of the ripples follow the crystallographic $<110>$ directions as shown in Fig. 4.13,

Fig. 4.14 summarizes our simulation results on crossover of morphologies between erosion and diffusion dominated structures. In KMC models the connection to time scales of experiments is made via the inter-event intervals which are proportional to inverse rates. In a typical experiment, fluxes are of the order of $7.5 \times 10^{15}$ ions $\mathrm{cm}^{-2} \mathrm{~s}^{-1}$ which corresponds to $\Phi \simeq 1 \mathrm{ion} /(\mathrm{atom} \cdot \mathrm{s})$. 
For a system of size $L \times L$, one diffusion step (one sweep over the whole lattice) is taken after $\Phi L^{2} / k_{1}$ erosion steps (shooting one ion). The default values for a system with $L=128$ lead to ratio of incidence interval to diffusion interval $\left(\mathcal{R}=\tau_{i} / \tau_{d}=0.1\right)$, which means one diffusion step is taken after shooting 12 ions. We increased this ratio up to 100, corresponding to $k_{1}=1.2 \times 10^{6} \mathrm{~s}^{-1}$. Snapshots of the evolving topographies for different values of $\mathcal{R}$ are shown in Fig. 4.14, A wide variety of morphologies from ripples following the ion-beam direction to smooth surfaces (from layer-by-layer erosion) are covered. The characteristic coarsening of structures reported in experiments (see section 2.1.1) is observed for $\tau_{i} / \tau_{d}=1,2$ and 5. (2nd, 3rd and 4th rows). A transition from ripples to pyramids occurs on longer times for $\tau_{i} / \tau_{d}=5$, whereas pyramids form directly from intermediate-time roughened surfaces for $\tau_{i} / \tau_{d}=10$. For high diffusion, fluctuations in height of surface do not exceed more than $\sim 1$ layer which is commonly observed in layer-by-layer regimes.

The roughness of morphologies shown in Fig. 4.14 are calculated and shown in Fig. 4.15 emphasizing the strong suppression of erosion (BH) and diffusion (ES) type instabilities for high diffusion rates. 


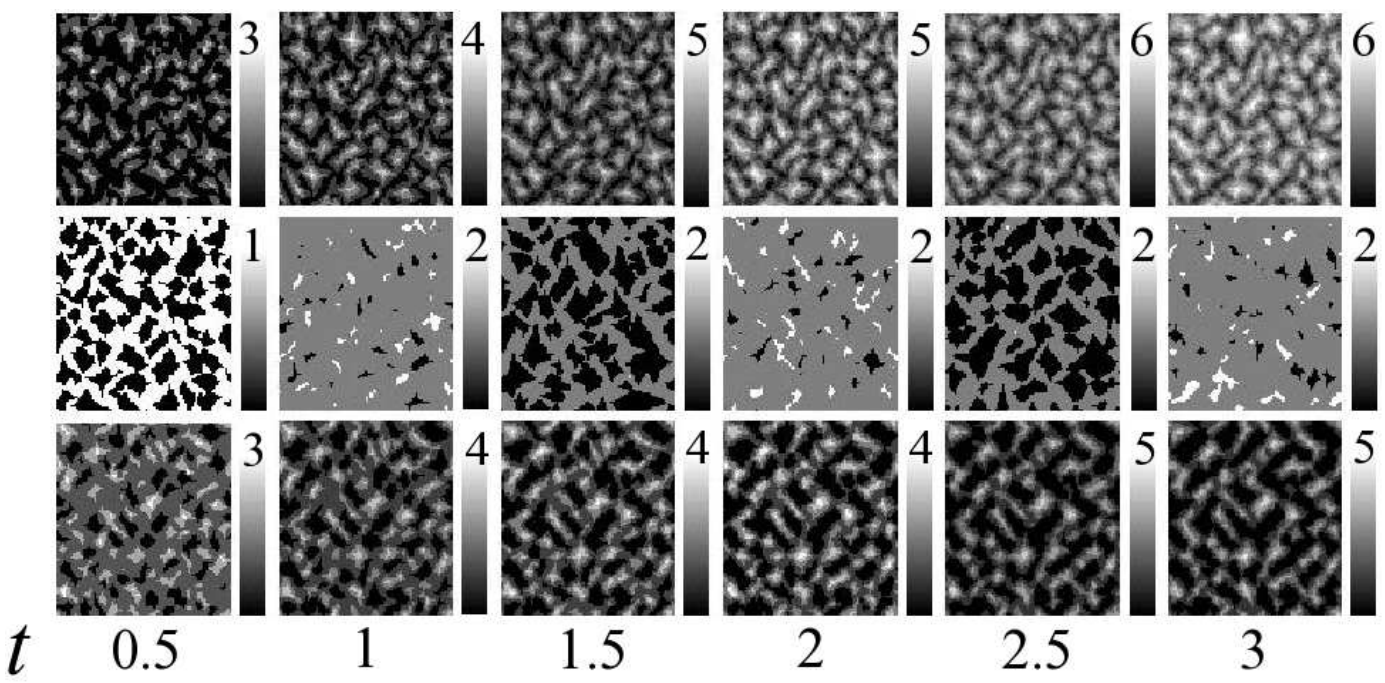

Figure 4.12: Evolution of clusters formed by upper row: adatoms, middle row vacancies and lower row: adatoms and vacancies together. The lateral size of the system $L=128$. The attempt frequency is set to $10^{6} \mathrm{~s}^{-1}$ which corresponds to a reduction of $\sim 10 \%$ in substrate bond energy $E_{\mathrm{s}}$. Formation of ES induced patterns, i.e. pyramids, is evident.

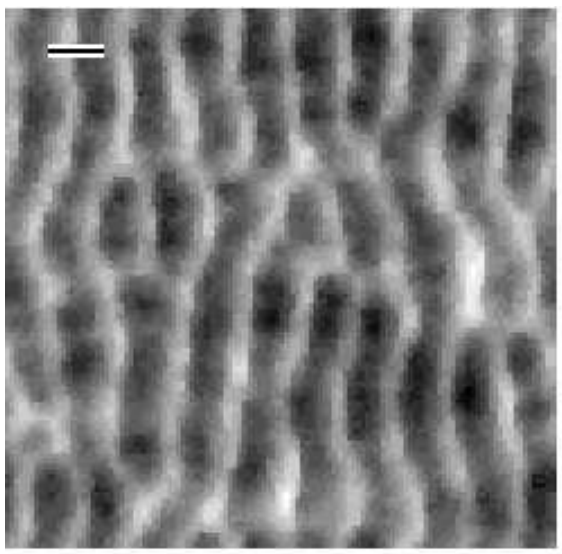

a
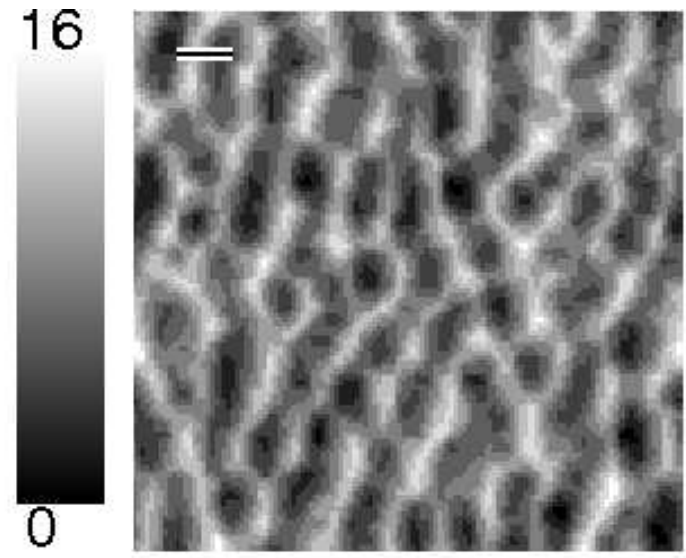

b

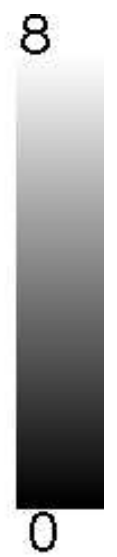

Figure 4.13: Surface profiles of a system with $L=128$ for $\theta=50^{\circ}, \phi=0^{\circ}$ at $t=3$ ions/atom and $T=0.01 \mathrm{eV}$. The bar indicates the azimuthal direction of ion-beam. Left: Arrhenius diffusion with default parameters for bond breaking and ES barrier, right: same as left, but with enhanced diffusion attempt rate. 


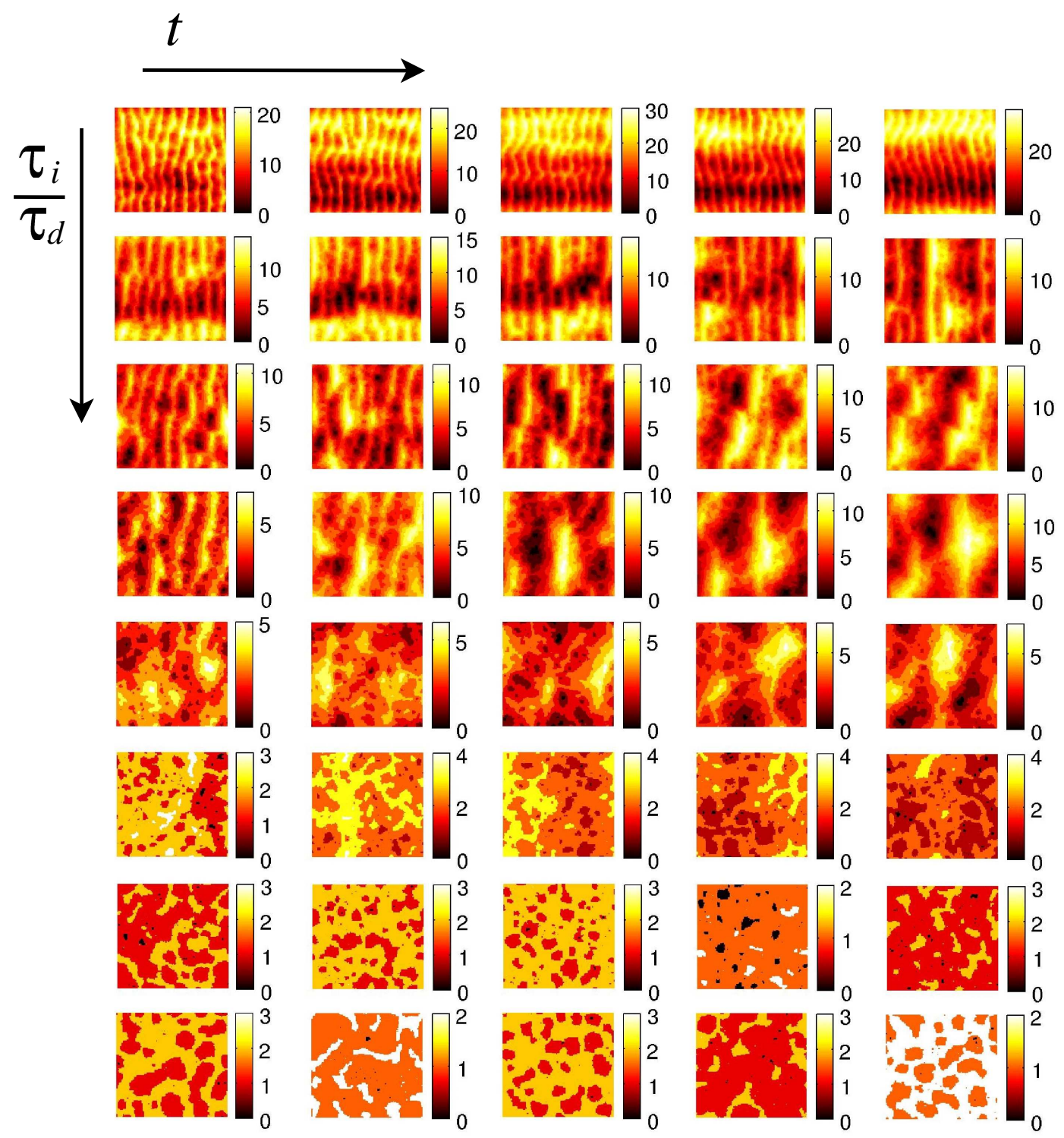

Figure 4.14: Surface profiles for different $\mathcal{R}=\tau_{i} / \tau_{d}$ increasing from up to down: 0.1, 1.0, 2.0, 5.0, 10, 20, 50 and 100 evolving in time. snapshots from left to right at $t=10,20,30$, 40 and 50. Temperature: $T=580 \mathrm{~K}$. 


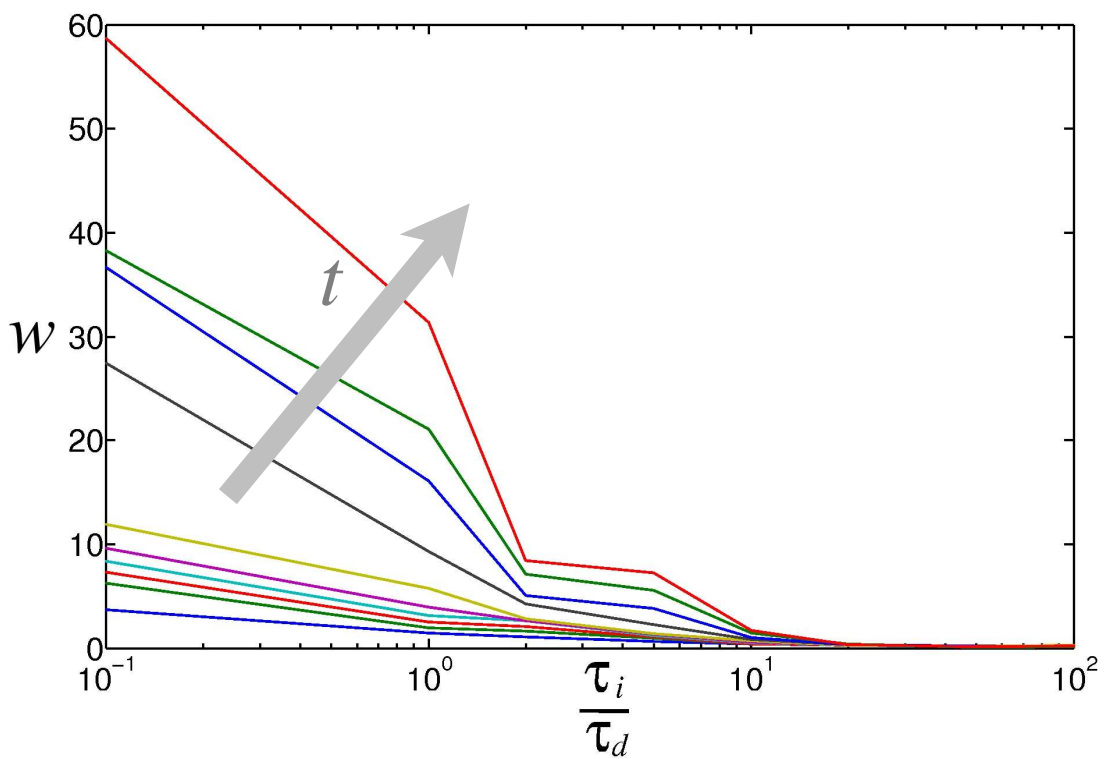

Figure 4.15: Corresponding roughness to the profiles depicted in Fig. 4.14, For small values of $\mathcal{R}$, the roughness increases with time with smaller rate for larger $\mathcal{R}$, wheres by increasing the diffusion rate, after reaching a certain value of $\mathcal{R}$, roughening is suppressed. 


\section{Chapter 5}

\section{Surfactant Sputtering}

Recently a novel sputter erosion technique has added further possibilities of fine-tuning processing conditions in many ways (Hofsäss and Zhang 2008, Hofsäss et al. 2009, Hofsäss and Zhang 2009). It prepares a sub-mono-layer coverage of the substrate surface (A) with "surfactant atoms" (B), which are constantly re-deposited by co-sputtering of a nearby surfactant (commonly metallic) target (see Fig. 5.1). We call the co-deposited particles surfactant (SURFace ACTive agENT) because it has been observed that the deposited atoms act as active agents to reduce (or amplify) the sputtering yield of substrate atoms. The effects of surfactants are not limited to the modulation of the sputter yield. Effects due to diffusion, clustering or mixing/demixing of the surfactants can be seen in different experiments under different ion-beam conditions and material combinations. Depending on diffusion, mixing and alloying properties of surfactant and target atoms, the surfactant distribution may either trend to form a homogeneously mixed A-B layer, or develop inhomogeneous patterns by mechanisms like surface segregation, island formation, clustering, diffusion instabilities or attachment to surface defects. The coverage by a surfactant density significantly changes the local sputtering yield of the substrate. In most cases the yield is reduced, though in exceptional cases it may also be increased (Berg et al. 1992). These local changes provide a feed-back mechanism between the pattern formation processes of substrate and surfactant atoms. By choosing appropriate surfactant-substrate combinations, a variety of surface patterns may be obtained in a controlled way. Furthermore, the surfactant distribution may itself become a technologically 


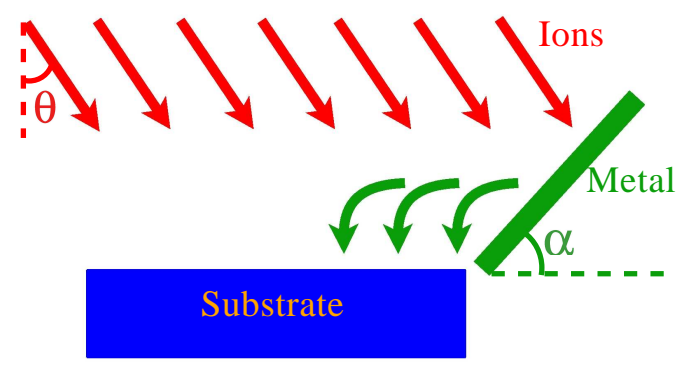

Figure 5.1: Surfactant sputtering experimental setup; A metallic layer is located beside the main sample and ion-beam targets the sample and the metallic layer simultaneously. Therefore metallic atoms are eroded and re-deposited on the sample. By varying the angle $\alpha$ the distribution of re-deposited metallic atoms can be tuned.

useful, self-organized structure, e.g. emergence of ordered arrays of dots or nanowires. The deposition current of surfactants $\mathbf{J}_{\mathrm{B}+}$ maintaining the average coverage during sputter erosion, is tuned beyond the re-sputtering limit, so that film growth of $\mathrm{B}$ is suppressed. This technique also allows to prepare spatial profiles of $\mathbf{J}_{\mathrm{B}+}$, so that modifications of the yield from nanometer to macroscopic length scales can be controlled.

We have set up both a Monte Carlo simulation model and a continuum theory of surfactant sputtering. Here, we will only consider the special case of demixing surfactant-substrate combinations and assume that surfactant atoms are the topmost of the surface provided by the substrate. In the following, we present the modeling of surfactants in our continuum theory and in KMC. Then we present results obtained for different set of parameters, corresponding to three scenarios of pattern formation.

\section{$5.1 \quad$ Implementation}

The continuum and the KMC approach are related in the sense that both are built upon the same basic physical mechanisms of erosion and surface diffusion. The description of erosion in both models are based on Sigmund's sputtering formula, and the continuum theory contains a large length-scale description of the surface diffusion of substrate and surfactant as implemented in the KMC model. But let us emphasize that in our KMC model, surfactant particles 
form a coverage (less that 100\%) on the top most layer of the system and no intermixing with substrate or island formation with heights more than 1 monolayer by surfactants is allowed. This restriction is not needed in continuum theory.

\subsubsection{Continuum theory}

The continuum description starts from the balance equation of mass within a sub-volume $V$ of the A substrate and its surface $\partial V$. The bulk density $\rho$ of the substrate is assumed to be constant, the surfactant is characterized by a varying surface density $\sigma$. If we denote the erosion velocity (normal to the surface) by $v_{n}$, the balance of substrate mass is expressed as $\rho v_{n}=-J_{A}-\nabla_{S} \cdot \mathbf{j}_{\mathbf{A}}$ with erosion current $J_{A}$ and surface diffusion current density $\mathbf{j}_{\mathbf{A}} \cdot \nabla_{S}$ denotes the surface divergence. The balance equation for $\sigma$ takes the form of

$$
\frac{D_{S} \sigma}{d t}=-J_{B}+\mathbf{J}_{r d} \cdot \mathbf{n}-\nabla_{S} \cdot \mathbf{j}_{B}
$$

Here, $\mathbf{n}$ denotes the outward normal unit vector of the surface. The transport derivative is given by Cermelli et al. (2005)

$$
\frac{D_{S} \sigma}{d t}=\partial_{t} \sigma+v_{n} \mathbf{n} \cdot \nabla \sigma-\sigma v_{n} \kappa
$$

It takes into account all the temporal changes of the surface morphology. Here, $\kappa=\nabla \cdot \mathbf{n}$ denotes the mean curvature of the surface.

The model is completed by expressing the currents and current densities in terms of the surface geometry and the surfactant density. We take the expressions for the erosion currents in the absence of surfactants from the $\mathrm{BH}$ linear and CB non-linear theories (see section 3.4). In addition, we take into account modifications of the sputtering yields so that the erosion currents of substrate and surfactant, $J_{A}$ and $J_{B}$, respectively, are given by

$$
J_{A}=J_{0} Y_{A} g_{A}(\sigma)\left(1-\nu_{B H}\right)
$$

and

$$
J_{B}=J_{0} Y_{B} g_{B}(\sigma)\left(1-\nu_{B H}\right) .
$$

$J_{0}$ denotes the flux of incident ions. $Y_{A}$ and $Y_{B}$ are the sputtering yields of the pure A and B system, respectively. We keep the first terms of the standard 
gradient expansion of the yield modification due to surface morphology,

$$
\nu_{B H}=v_{0}^{\prime} \cdot \nabla h+\nu_{x} \frac{\partial^{2} h}{\partial x^{2}}+\nu_{y} \frac{\partial^{2} h}{\partial y^{2}}+\frac{\lambda_{x}}{2}\left(\frac{\partial h}{\partial x}\right)^{2}+\frac{\lambda_{y}}{2}\left(\frac{\partial h}{\partial y}\right)^{2} .
$$

The factors $g_{A}$ and $g_{B}$ are in the form of $g_{A}(\sigma)=\max [1-\lambda \sigma, 0]$ and $g_{B}(\sigma)=\sigma$ to parametrize the changes of sputtering yields due to small $\mathrm{B}$ coverages. This choice is in accordance with the experimental findings of Hofsäss and Zhang (2008) for small surfactant densities. Note that the non-linearities in $\nu_{B H}$ may be kept up to any desired order, but we have to keep the full geometrical non-linearities in the transport derivatives, because otherwise we would violate mass conservation during surface diffusion.

The surface diffusion current densities $\mathbf{j}_{\mathbf{A}}$ and $\mathbf{j}_{\mathbf{B}}$ contain near-equilibrium and non-equilibrium terms, which are driven by the reduction of surface free energy and the external erosion and re-deposition fluxes respectively. Here, we only take into account simple contributions arising from expansions in $\nabla h$ and $\sigma$ and a simple, phenomenological expression for the non-equilibrium EhrlichSchwoebel (ES) current (see section. 4.2.4), so that for the simplest case of isotropic (amorphous) samples

$$
\nabla_{S} \mathbf{j}_{\mathbf{A}} \approx K_{1}\left(\nabla^{2}\right)^{2} h+K_{A, E S} \nabla^{2} h\left(1-\ell_{d}^{2}(\nabla h)^{2}\right)
$$

and

$$
\nabla_{S} \mathbf{j}_{\mathbf{B}} \approx-\nabla\left(D_{B} \nabla \sigma\right)+K_{B, E S} \sigma \nabla^{2} h\left(1-\ell_{d}^{2}(\nabla h)^{2}\right)
$$

Continuum models, which also consider the time evolution of densities in a surface layer have appeared in the literature, which differ in important aspects from the present work. In the work of Shenoy et al. (2007), a bulk binary alloy is considered, and in the work of Aste and Valbusa (2005) and Castro et al. (2005) a layer of adatoms of the target material is included. Both papers also differ from the present work in the physical concepts, which underlie the evolution equation of the surface density.

In the numerical solutions presented below, we have extended this simplest diffusion model in two ways: (i) we explicitly took into account a cubic anisotropy arising from eroding a (100) surface. (ii) We let B atoms cluster by putting $D_{B} \propto \max (1-c \sigma, 0)$. This has been done to facilitate comparisons with our Monte Carlo simulations, which naturally include these effects. The 
system of non-linear partial differential equations, which make up the continuum model have been solved by a finite element method. We used a linear implicit Euler time stepping algorithm and $\mathrm{C}^{1}$ finite elements on a triangular grid. Similar solver has been set up for MBE by Burger (2006). We have implemented the algorithm using the free software FEM package FreeFEM ++1

\subsubsection{KMC model}

We modified our KMC model to include surfactant atoms and their effects on sputtering and diffusion of substrate atoms as the following. Each surface site is occupied either by a substrate atom (A) or by a surfactant atom (B). The sputtering probabilities for $\mathrm{A}$ and $\mathrm{B}$ atoms may be different, but note that only surface atoms are sputtered off. Thus, a B atom at $\mathbf{r}$ suppresses the A sputter yield at this site completely. In addition, a B atom may also reduce the sputter yield of $\mathrm{A}$ atoms at a nearest neighbor site by a factor $1-\Lambda$; $0 \leq \Lambda \leq 1$. B atoms, which are sputtered off are immediately replaced via random re-deposition. The implemented diffusion model is the thermally activated Arrhenius model with net-bound-breaking barriers (see section 4.2.3). In principle, binding energies between surfactant atoms $E_{B B}$ and substrate atoms $E_{A A}$, and also the energy of inter-species bindings $E_{A B}$ may be different. The Ehrlich-Schwobel barrier is only for A atoms taken into account. We used the default values for bound energies of substrate atoms and vary $E_{A B}$ from $0 \cdots E_{A A}$ and $E_{B B}$ from $E_{A A} \cdots 0.6 \mathrm{eV}$. We have studied B yields from $1 \cdots 0.1$ times the A yield. Temperature is set to $T=600 K$. In the following sections, different sets of parameters, corresponding to different experiments setups are presented.

\section{Identical surfactants}

Here, as the simplest case, we present some results considering surfactants with the same sputtering yield as substrate atoms and the same binding energies for all tree types of bindings. (Note that there is still a difference between $\mathrm{B}$ and $\mathrm{A}$ atoms arising from re-deposition of a B atom as it is sputtered and also the inhibition of the jumps over B atoms.) In Fig. 5.2, profiles sputtered

\footnotetext{
${ }^{1}$ www.freefem.org
} 

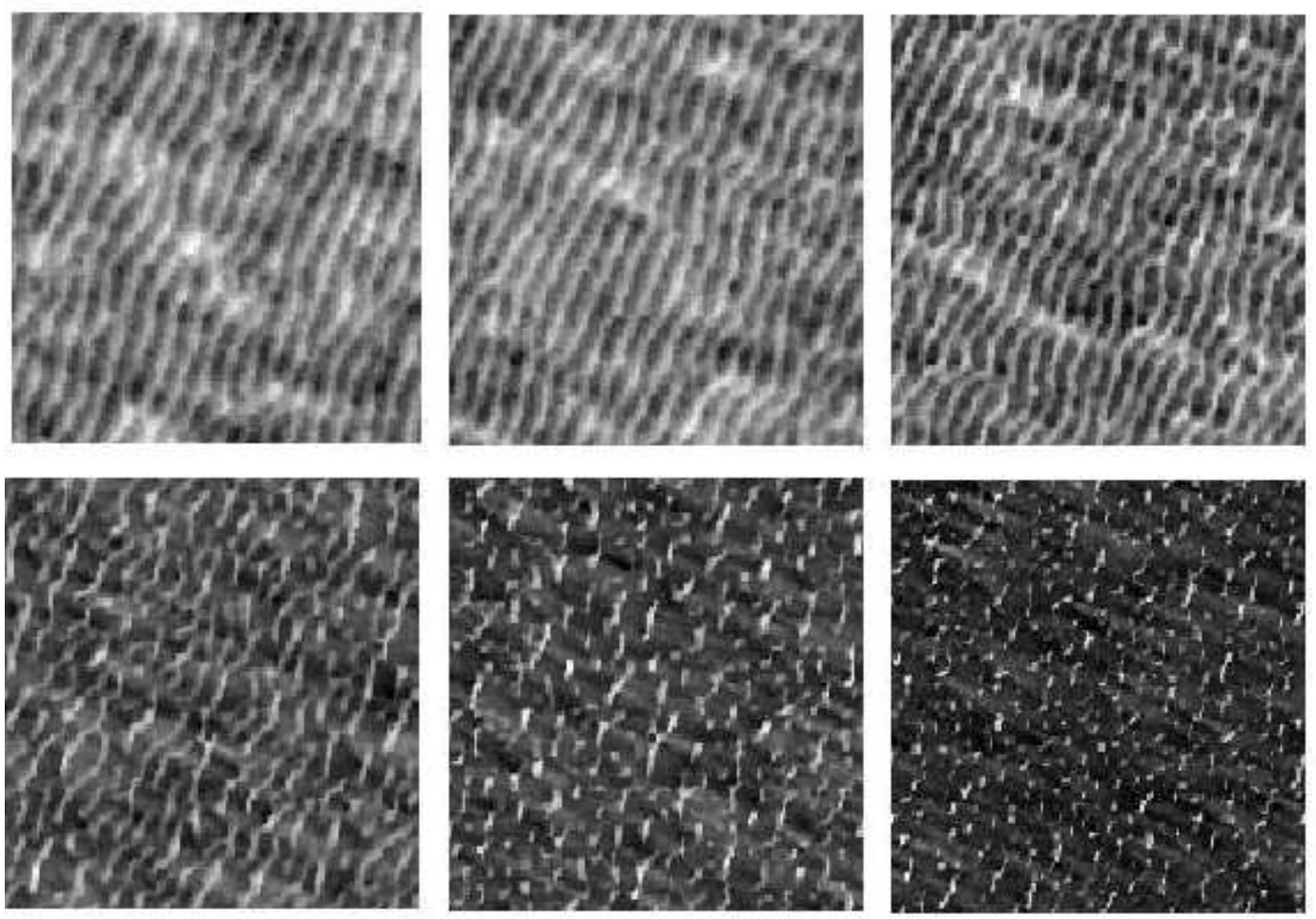

Figure 5.2: Morphologies evolved after 3 ions/atom sputtering, with different coverage (from left to right and up to down: $c_{s} 0.00 .10 .30 .50 .70 .9 \%$ ) of surfactants $B=A$. The ripples blurred as the coverage is increased.

for $t=3$ ions/atom in different coverages of $\mathrm{B}$ atoms are depicted. As the coverage increases, the ripples get shorter in length and the typical wavelength of the ripples slightly decreases (shown in Fig. 5.3). The later is expected from BH model where less diffusivity of particles leads to smaller wavelength of the patterns. We also calculated the roughness of surface for different coverages (Fig. 5.4). Although the periodic BH patterns are annihilated by increasing the coverage, the total roughness of the surface increases.

\subsection{Mesoscopic height gradient}

As mentioned above, an experiment setup as introduced in Fig. 5.1 produces an inhomogeneous spatial distribution of $\mathbf{J}_{\mathrm{B}+}$, which is close to a constant gradient so that it leads to a linearly decreasing coverage of B. Here, we set 


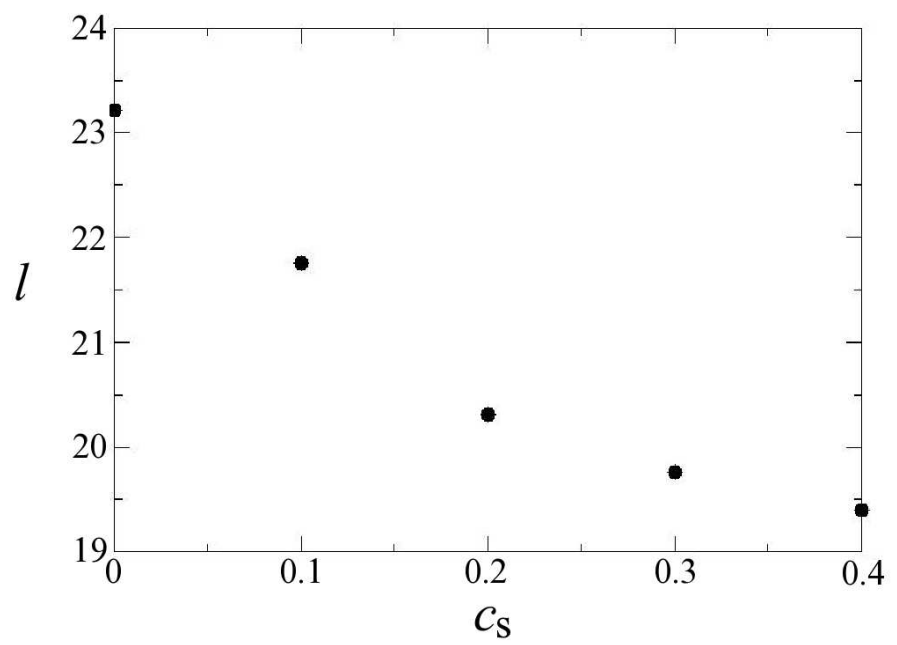

Figure 5.3: The characteristic wavelength of patterns depicted in Fig. 5.2 as a function of surfactant coverage. The wavelength is measured in lattice constant unit by using PSD method.

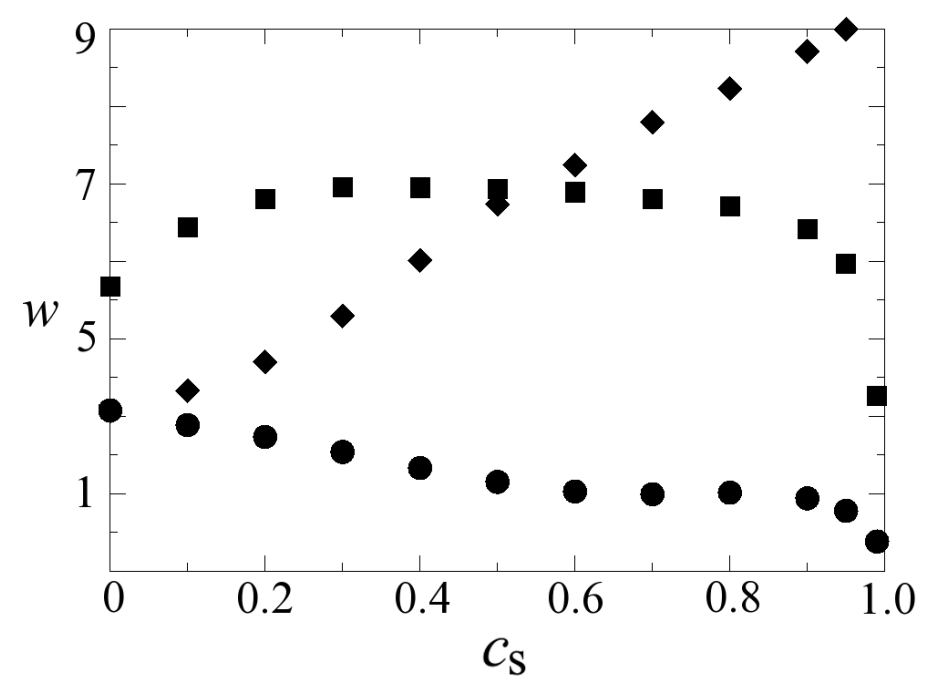

Figure 5.4: Roughness of surface against coverage with surfactant. diamonds: $\mathrm{A}=\mathrm{B}$, circles: Sputtering yield $Y_{B}$ is $10 Y_{A}$ and the A yield is suppressed by $0.25 Y_{A}$ from every nearest neighbor B atom, squares: Clustering of $\mathrm{B}$ and demixing favored, $E_{A A}=0.18, E_{A B}=$ $0, E_{B B}=0.6$. Surface diffusion without ES barriers. 
(a)

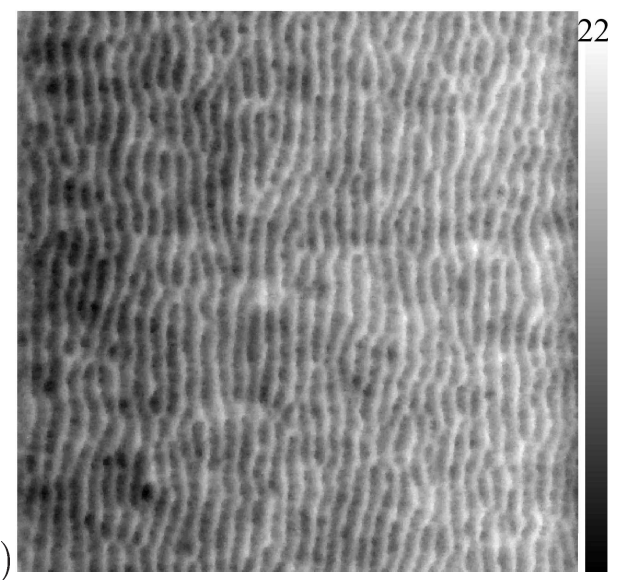

(b)

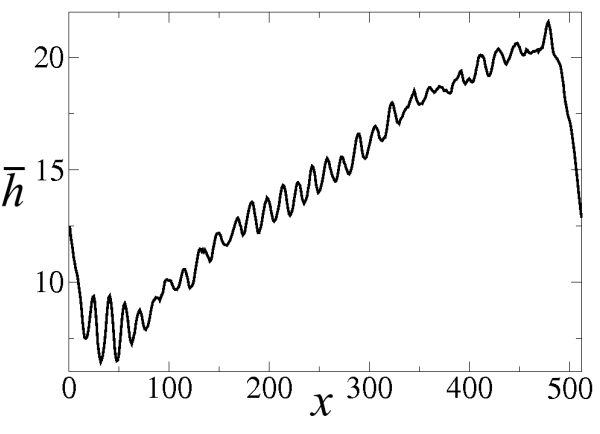

Figure 5.5: Mesoscopic height gradient by surfactant sputtering; (a) surface profile after 3 ions/atom sputtering with an inhomogeneous surfactant coverage with average of $20 \%$. Other parameters are as in Fig. 5.4 (circles). (b) Mean of surface height in direction perpendicular to ion-beam direction. Concurrent to the formation of ripples, a global slop is also induced along the sample length.

a smaller sputtering yield of $Y_{B}=0.1 Y_{A}$ for B atoms and the same diffusion barriers for A and B. The average B-coverage is $20 \%$ and it decreases linearly along $x$ direction. The maintained structure is shown in Fig. 5.5(a). Surprisingly, a slight coverage of surfactants modifies the common BH ripples with nanometer length-scales with a defined slope over micrometer length-scales (see Fig. 5.5(b)).

\subsection{Morphology modification}

In addition to the large scale modifications on standard $\mathrm{BH}$ ripples, presented in the previous section, surfactant sputtering is also a proper method to manipulate the pattern formation via IBS at the scale of the standard patterns, i.e. nanometers, and produce variations in patterns types. In the following, three different scenarios for such changes in morphology of patterns are presented.

\subsubsection{Ultra-smooth surfaces}

As demonstrated in Fig. 5.6, a strong suppression of the substrate sputter yield due to surfactant coverage may lead to very smooth surfaces, reminiscent of 
layer-by-layer erosion, instead of rippled topographies. The parameters used here are $\Lambda=0.25$ in KMC and $\lambda=2$ in continuum theory for a coverage of only $20 \%$ of surfactants. The diffusion parameters are still the same for both types. We started the numerical solution with an initially rough surface and observed a monotonous decrease of surface roughness.

Using the KMC model, we studied the profiles patterning for different coverages of surfactants. For the fix sputtering time, a suppression of pattern formation by increasing the coverage is observed (profiles depicted in Fig. 5.7). The surface roughness for different coverages is shown in Fig. 5.4. Roughness of the surface decreases monotonically as the surfactant coverage increases. This case has correspondence to the experiments of co-sputtering of $\mathrm{Au}$ on $\mathrm{Si}$ by Hofsäss and Zhang (2008).

\subsubsection{Arrays of nano-clusters}

In this part, the barriers have been changed to facilitate B-clustering and favor demixing $\left(E_{A B}=0, E_{B B}=0.6\right) \mathrm{eV}$. The obtained profiles and distribution of $\mathrm{B}$ atoms are shown in Fig. 5.8. The strong clustering tendency causes a highly significant redistribution of the surfactant B on the A surface. The majority of $B$ atoms would be located in valleys of the ripple topography if they were distributed randomly (due to the morphology of the ripples). In Fig. 5.9, we show the ratio of the number of $\mathrm{B}$ atoms to the number of randomly distributed atoms, which constitutes a statistical estimate of surfactant surface density vs. height $h$ measured from the lowest point on the surface. Note that sputtering plus re-deposition of $\mathrm{B}=\mathrm{A}$ atoms only leads to a minor increase of density in valleys, whereas $B$ atoms strongly prefer to assemble on crests of ripples if they cluster and demix from substrate A atoms. In fact, one would expect a generic uphill current of any surfactant which suppresses the yield, because it is sputtered preferably from valleys (Bradley-Harper mechanism) but redeposited randomly. Thus, material is moved out of valleys. This behavior has been observed in experiments of Hofsäss and Zhang (2008) using a Si substrate and Ag surfactant. An improved control of this clustering could open up a way to efficiently fabricate regular arrays of quantum wires.

Note that if the clustering of surfactants and also the reduction of yield caused by them are strong enough, the BH ripple forming fails and what con- 

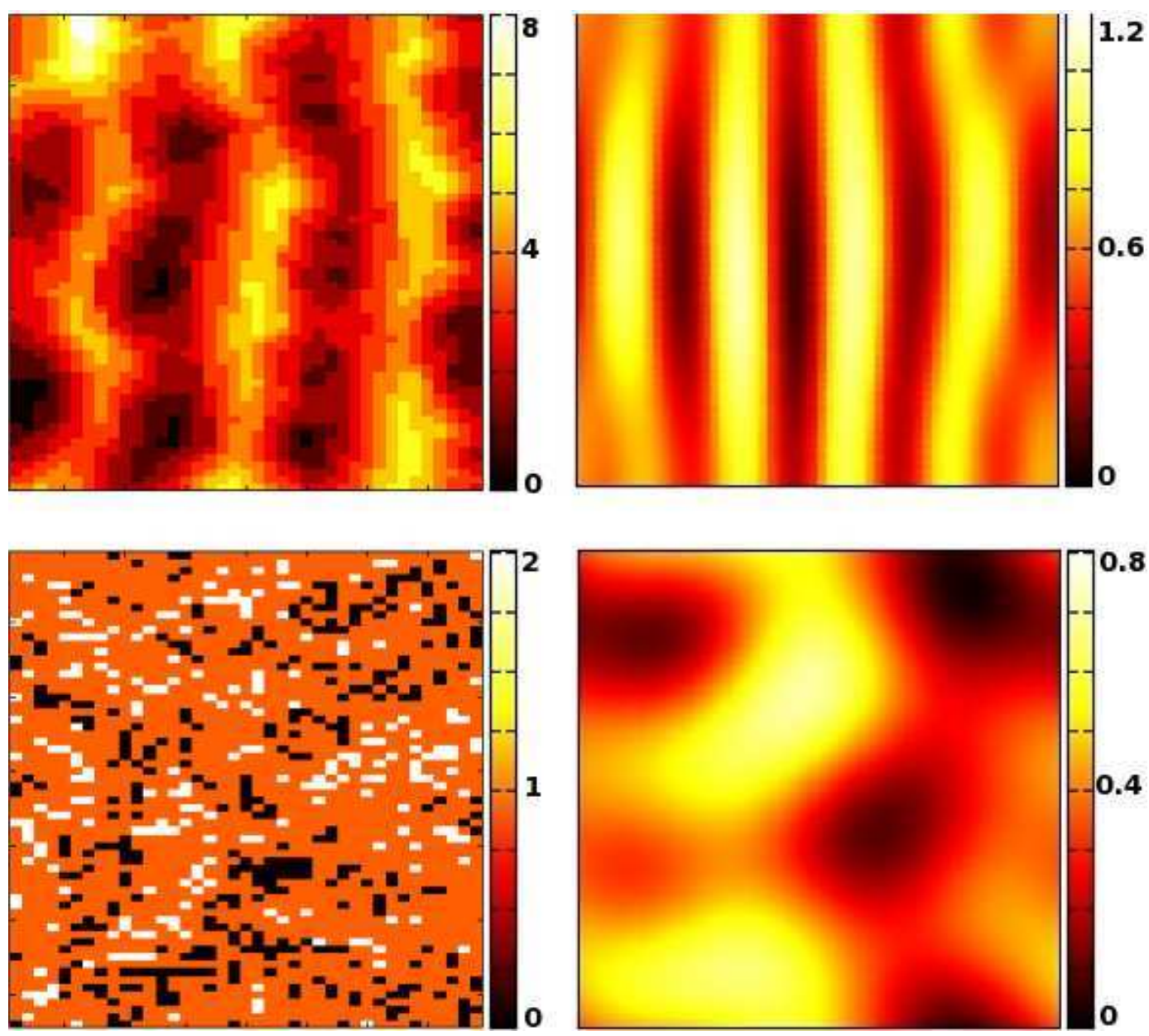

Figure 5.6: Upper panels: BH-type ripples without surfactant, left: KMC simulation, right: Continuum theory. Lower panels: Strong suppression of sputter yield of substrate by surfactant leads to smooth surfaces, left: $\Lambda=0.25$ in KMC, right: $\lambda=2$ in continuum theory. Here, the height scale is enhanced by a factor 100 with respect to the corresponding upper panel. 

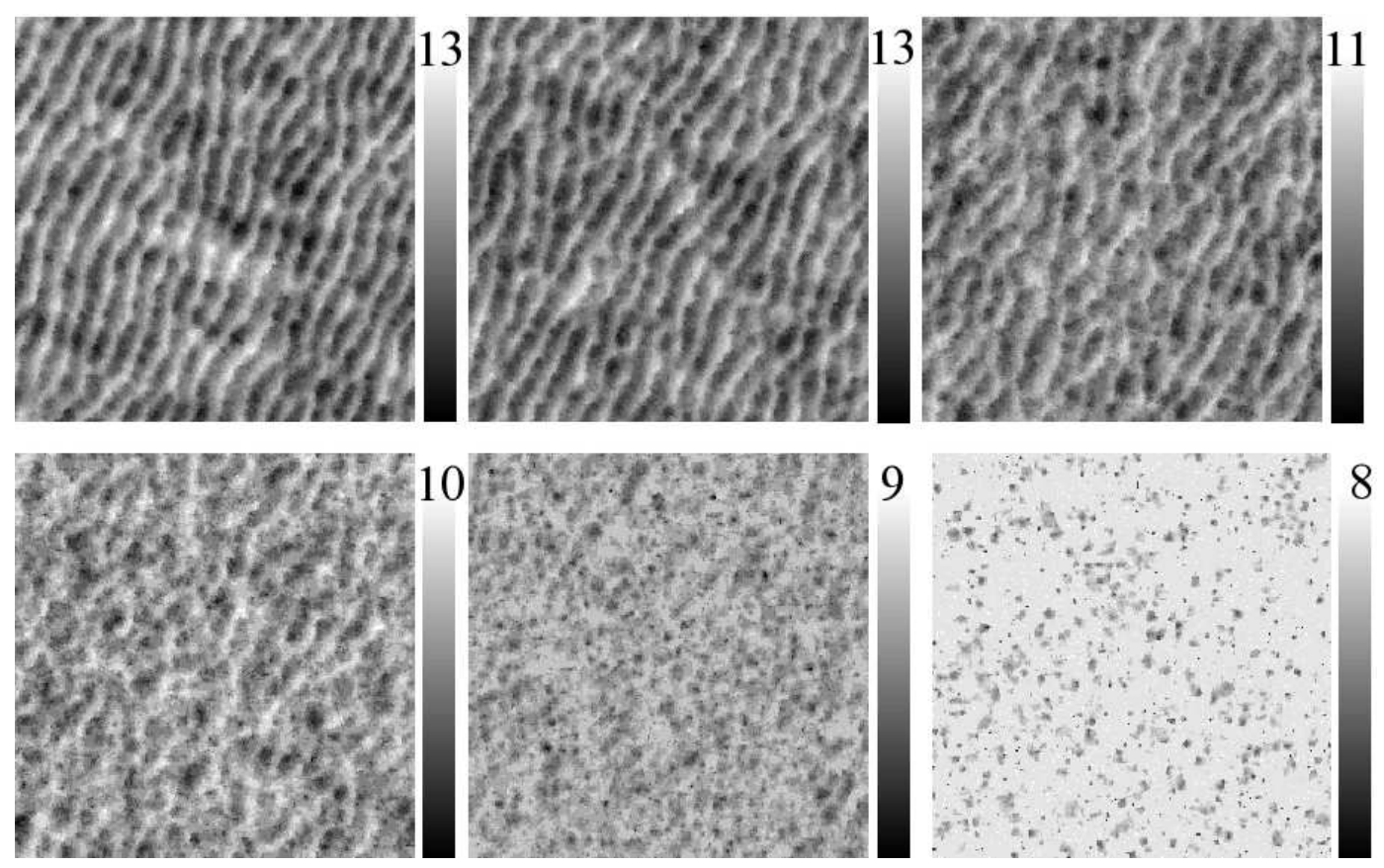

Figure 5.7: Morphologies evolved after 3 ions/atom sputtering with different coverages (from left to right and up to down: $c_{s} 0.00 .10 .30 .50 .70 .9 \%$ ) of surfactants. The parameters are as in Fig. 5.4 (circles). For high surfactant coverages, pattern formation is suppressed. 
trols the evolution of surface fluctuations are the distribution and morphology of surfactant clusters. This is discussed below.

\subsubsection{ES induced patterns}

The third scenario demonstrates how dot-like patterns due to Ehrlich-Schwoebel diffusion can be generated by a surfactant. As depicted in Fig. 5.10, without surfactant, the growth of ripples is the dominant process and typical BradleyHarper ripples emerge even in the presence of Ehrlich-Schwoebel diffusion. Coverage with a surfactant tends to suppress the Bradley-Harper instability and Ehrlich-Schwoebel diffusion can become the dominant, pattern-forming mechanism. Note that the ES-type dots can form an ordered array. Ordering is supported, if preliminary ripple structures break up into dots. Ordered dots are more clearly visible in the continuum theory. The KMC dynamics has been limited to the erosion of 5 monolayers to keep it consistent with the calibrated time in the continuum theory, but the effects of noise are too strong to detect dot ordering in KMC within this time interval. 

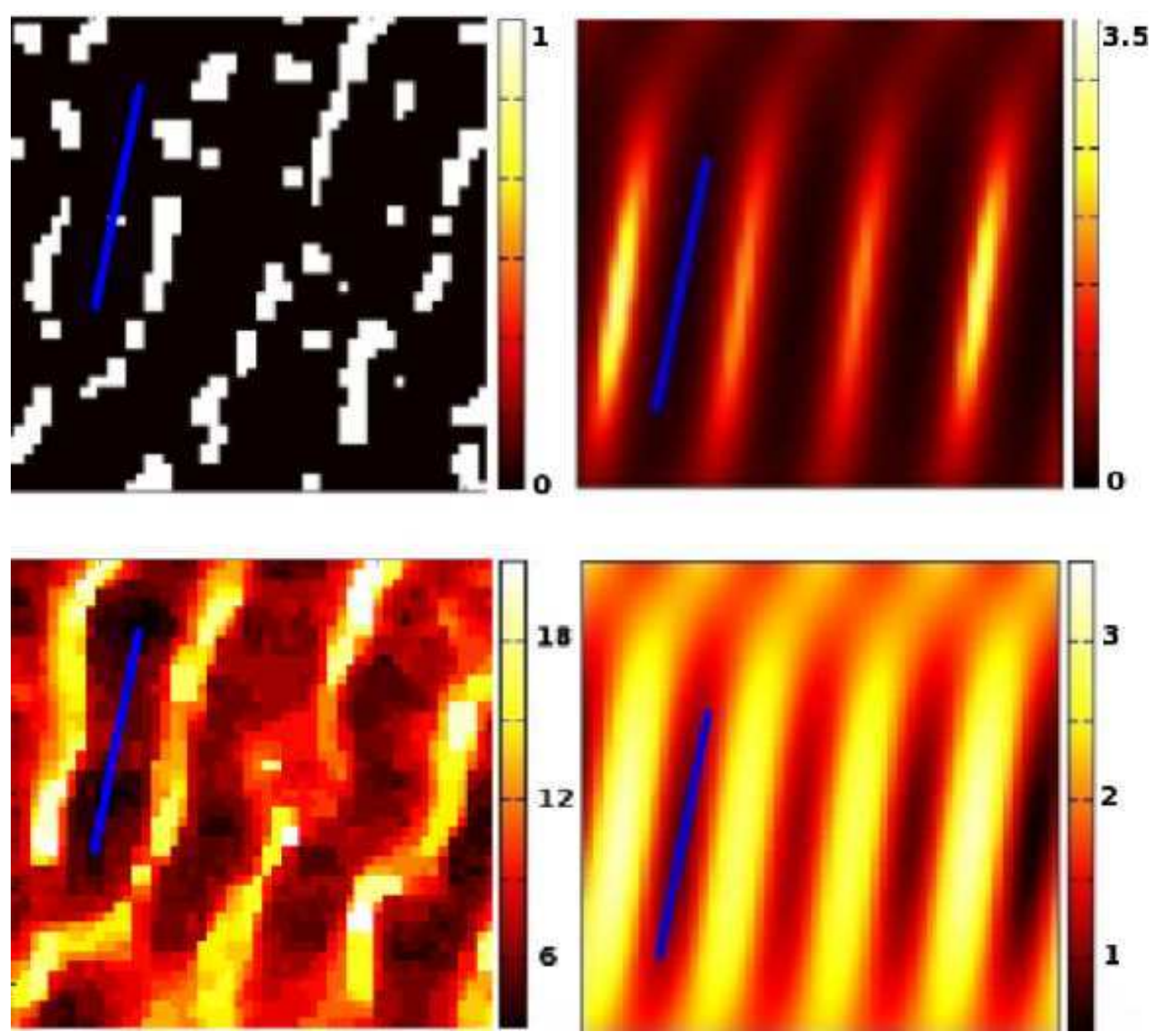

Figure 5.8: Lower panels: Ripple pattern of a substrate with 0.4 mono-layer coverage of surfactant with $Y_{A}=Y_{B}$ and strong clustering after 5 ions per surface atom, Left: KMC simulation $E_{A B}=0, E_{B B}=0.6 \mathrm{eV}$, Right panel: Continuum theory $(c=2)$. The direction of the ion-beam is inclined by $\phi=0.2$ with respect to the $\mathrm{x}$-axis. No Ehrlich-Schwoebel effects are included. The bars mark identical regions in upper and lower panels, respectively. Upper panels: Corresponding distributions of surfactant. 


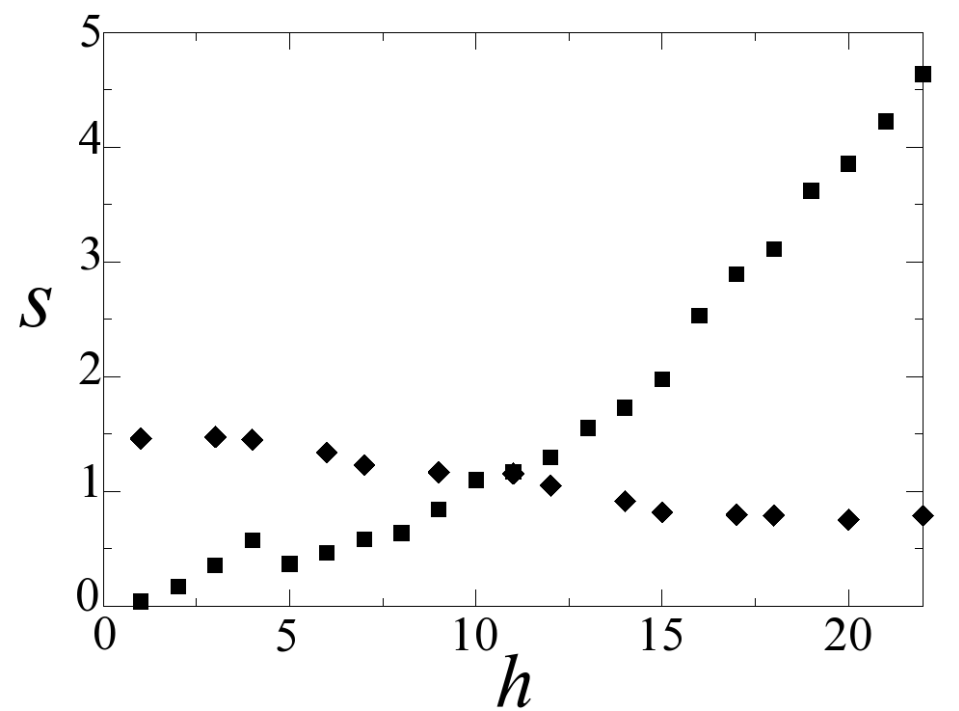

Figure 5.9: Statistical estimate of surface density of surfactant vs. height. diamonds: substrate species $\mathrm{A}=$ surfactant species $\mathrm{B}$, squares: surfactant $\mathrm{B}$ is clustering and demixing from A, similar to Ag on Si. 

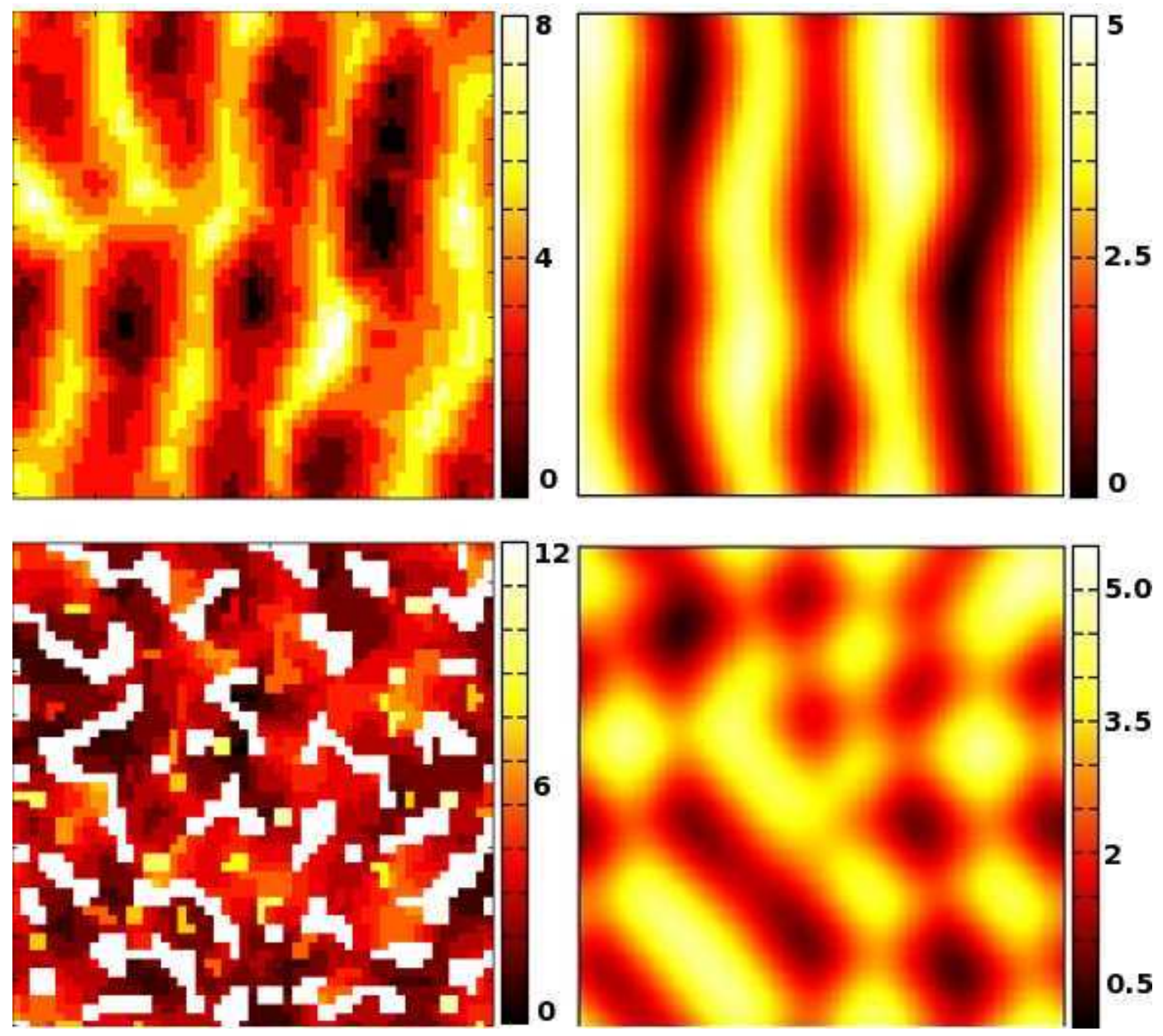

Figure 5.10: Upper panels: ES diffusion of substrate and surfactant is chosen such that BH instability dominates. No suppression of substrate sputter yield by surfactant. Lower panels: Moderate suppression of substrate sputter yield by surfactant $(\Lambda=0.1$ in KMC, $\lambda=0.6$ in continuum)tends to weaken the BH instability, so that ES diffusion can influence the pattern formation. Left panels: KMC simulation, Right panels: Continuum theory. 


\section{Chapter 6}

\section{Beam-noise induced effects}

In IBS, the randomness in the location of impact points of single ions is the source of intrinsic noise in etching process: "shot noise". In addition to the shot noise, other sources of external fluctuations may be present in an IBS setup. One example of this type of noise sources is fluctuation within the ion-beam. It is reported that optical properties of a beam like beam profile, which is the angular distribution of trajectories of ions around the mean direction of the ion-beam, is important to maintain different topographies with unexpected features and high regularity patterns (Ziberi et al. 2004; Ziberi et al. 2008) 1 Therefore we investigate the generic (non-material specific) effects of such external noise in IBS, using continuum theory and KMC simulation.

We classify fluctuations within the ion-beam into three types (see Fig. 6.1).

(i) Homogeneous sub-beams: In this case, the beam consists of identical groups of sub-beams originating from different parts of the beam. In each group, the direction of sub-beams $\mathbf{m}$ has a distribution $p(\mathbf{m})$. We assume that $p(\mathbf{m})$ is localized around the average direction $\langle\mathbf{m}\rangle=\mathbf{m}_{\mathbf{0}}$. The erosion velocity at an arbitrary point at the surface, induced by impinging ions, is the sum of contributions from all sub-beams weighted with $p(\mathbf{m})$.

(ii) Temporally fluctuating homogeneous beam: In this case, trajectory of all ions are parallel, but in a direction which changes with time stochastically, with the average of $\langle\mathbf{m}(t)\rangle=\mathbf{m}_{\mathbf{0}}$.

\footnotetext{
${ }^{1}$ Very recently it became clear that most of the reported effects are strongly material specific and dramatic changes of shape of patterns are due to interference of co-deposited Fe atoms with the standard scenarios of pattern formation. This kind of effects are extensively discussed in chapter 5 .
} 


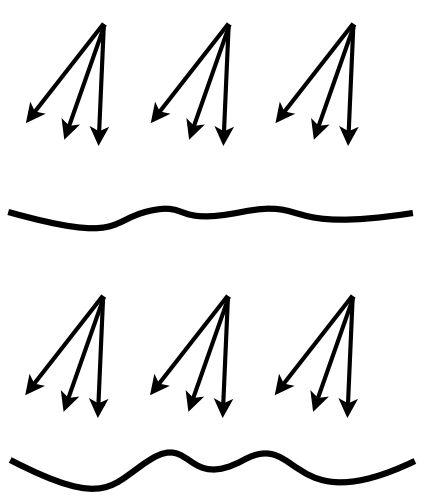

(i)

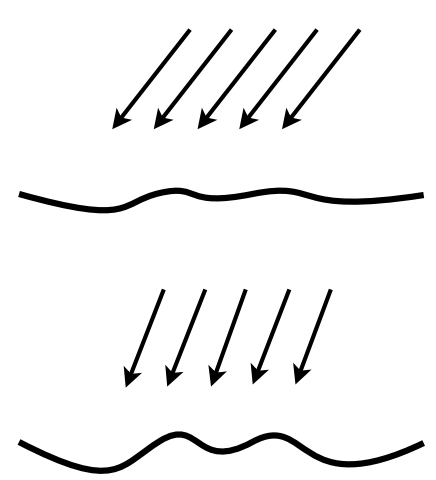

(ii)
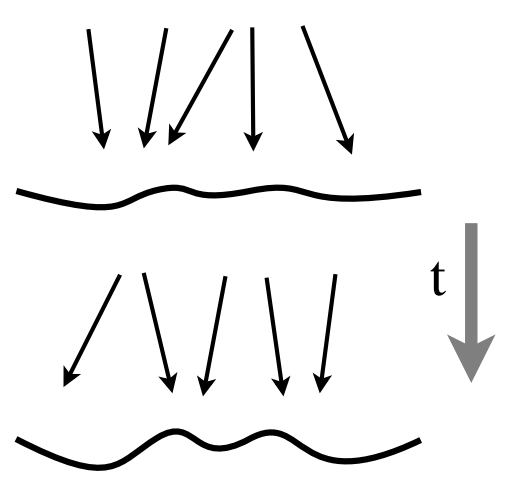

(iii)

Figure 6.1: Classification of beam-noise in three types: (i) Homogeneous sub-beams which are made up of identical sub-beams. Each sub-beam contains an ensemble of ions trajectories with direction distribution of $p(\mathbf{m})$. (ii) Temporally fluctuating homogeneous beam which includes ions traveling parallel but in a direction fluctuating in time. (iii) Spatio-temporally fluctuating beam, in which the direction of each ion trajectory is taken from a stochastic (in time and position) field.

(iii) Spatio-temporally fluctuating beam: This is the most general situation, in which every single ion takes its direction from a stochastic homogeneous field of unit vector $\mathbf{m}(x, y, t) \cdot \mathbf{m}_{\mathbf{0}}$, the ensemble average of $\mathbf{m}$ is constant in time.

For simplicity, we consider spatial fluctuations only in polar angle of incidence $\theta$, i.e. fluctuations in azimuthal angle are neglected here. We consider three types of distributions:

(a) a flat distribution centered at $\theta_{0}$ with the width of $\Delta \theta$,

(b) a Gaussian distribution with the standard deviation of $\Delta \theta$ from $\theta_{0}$, and

(c) a Gamma distribution which is fitted to the histograms of simulated ion-beam profiles using the data from experiments by Ziberi et al. (2008) (see Fig 6.2).

We also assume that in cases (ii) and (iii), correlation times of $\theta$ are so small that we can apply the white noise limit, i.e. $\left\langle\delta \theta(\mathbf{r}, t) \delta \theta\left(\mathbf{r}^{\prime}, t^{\prime}\right)\right\rangle=C\left(\left|\mathbf{r}-\mathbf{r}^{\prime}\right|\right) \delta(t-$ $t^{\prime}$ ) (for case (ii), $C$ is just a positive constant). We, furthermore, restrict our model to changes of $\delta \theta(\mathbf{r}, t)$, which are restricted to length scales larger than the shape parameters $\sigma$ and $\mu$ of the energy deposition function, so that in the 
(a)

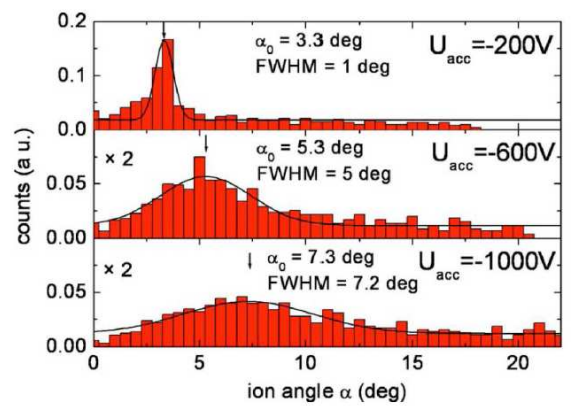

(b)

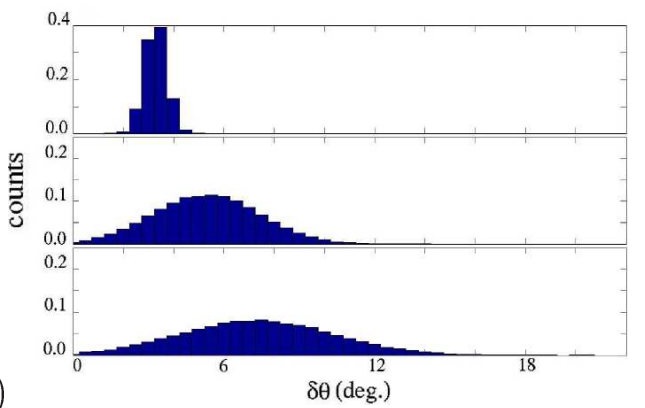

Figure 6.2: Simulated beam profiles (a) beam profiles simulated by Ziberi et al. (2008) for three different acceleration voltage. (b) Gamma functions fitted to the data of (a), and used as an input for KMC simulations.

gradient expansion the leading order terms take on the form

$$
\frac{\partial h}{\partial t}=-v_{0}(\theta(\mathbf{r}, t))+v_{0}^{\prime}(\theta(\mathbf{r}, t)) \frac{\partial h}{\partial x}+\nu_{x}(\theta(\mathbf{r}, t)) \frac{\partial^{2} h}{\partial x^{2}}+\nu_{y}(\theta(\mathbf{r}, t)) \frac{\partial^{2} h}{\partial y^{2}}-K \nabla^{4} h
$$

and $\nabla \theta$-terms can be neglected in case (iii). We expand in $\delta \theta$ up to linear order and obtain

$$
\frac{\partial h}{\partial t}=-v_{0}\left(\theta_{0}\right)-v_{0}^{\prime}\left(\theta_{0}\right) \delta \theta+\hat{L}_{0} h+\delta \theta \hat{L}_{1} h
$$

with $\hat{L}_{0}=v_{0}^{\prime} \partial / \partial x+\nu_{x} \partial^{2} / \partial x^{2}+\nu_{y} \partial^{2} \partial y^{2}-K \nabla^{4}$. Using techniques of small noise expansion (Garcia-Ojalvo and Sancho 1985) for multiplicative noise, we now derive closed equations for $\langle\tilde{h}\rangle=\langle h\rangle-v_{0} t$ from

$$
\frac{\partial\langle\tilde{h}\rangle}{\partial t}=\hat{L}_{0}\langle\tilde{h}\rangle+\left\langle\delta \theta \hat{L}_{1} \tilde{h}\right\rangle
$$

Note that the operator $\hat{L}_{1}=\partial \hat{L}_{0} /\left.\partial \theta\right|_{\theta=\theta_{0}}$ has the same form as $\hat{L}_{0}$, if the parameters $v_{0}, \nu_{x}$ and $\nu_{y}$ are replaced by $v^{\prime}=\partial v_{0} / \partial \theta, \nu_{x}^{\prime}=\partial \nu_{x} / \partial \theta$ and $\nu_{y}^{\prime}=\partial \nu_{y} / \partial \theta$ respectively.

\subsection{Homogeneous sub-beams}

In this case (i), we can directly calculate the average of height $\langle\tilde{h}\rangle$ considering averages of independent Fourier mode solutions of Eq. 6.3 over $\theta$, i.e. $\langle\tilde{h}(\mathbf{k}, t)\rangle=\left\langle e^{\hat{L}(\mathbf{k}, \theta) t}\right\rangle h(\mathbf{k}, 0)$. 
(a)
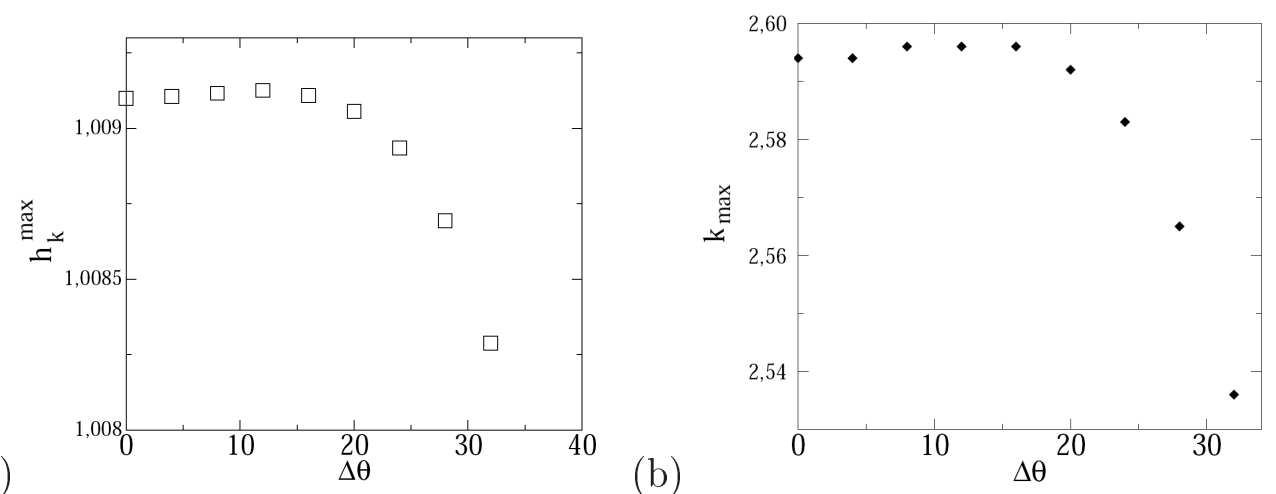

Figure 6.3: Homogeneous sub-beams; (a) The height of fastest growing mode for different beam-divergence width. Growth of the fastest mode is decelerated by increasing the noise. (b) The wavenumber of the fastest growing mode for different beam-divergence width. A slight change in the emerging wavelength is predicted.

For the Gaussian distribution (b), averaging $\exp \left(\delta \theta L_{1}(\mathbf{k}) t\right)$ shows that the growth law deviates dramatically from the exponential form of the non-fluctuating case and follows the form $\langle\tilde{h}(\mathbf{k} t)\rangle \propto \exp \left(\left\langle\delta \theta^{2}\right\rangle L_{1}(\mathbf{k})^{2} t^{2} / 2\right)$. This means that we are already out of the range of validity of linear approximation. For the flat distribution (a), again an exponential growth does not satisfy the growth equation. After a transient time, the growth takes on the form $\propto t^{-1} \exp (r t)$.

We have also performed numerical evaluations of ensemble averages. Fitting an exponential growth function to the averaged height $\langle\tilde{h}\rangle$, one can extract an effective growth rate. These effective rates calculated for case (a) are shown in Fig. 6.3 (a). For small fluctuation, the effective rate increases slightly above its original value without any beam-noise. Increasing the beam-divergence beyond $\Delta \theta \approx 12^{\circ}$ reduces the rate and slows down the formation of patterns. The decrease of rate becomes more obvious for divergences larger than $\Delta \theta \approx 20^{\circ}$. Another outcome of beam-noise, shown in Fig. 6.3 (b) is a slight change in the wavelength of the fastest growing mode.

\subsection{Temporally fluctuating homogeneous beams}

As mentioned above, in the case of temporally fluctuating homogeneous beams, we assume that there is no correlation in time in the stochastic time series of the 
direction of beam. Using Novikov's theorem, the averaged first-order growth equation, $\partial_{t}\langle\tilde{h}\rangle=\hat{L}_{0}\langle\tilde{h}\rangle+\left\langle\delta \theta \hat{L}_{1} \tilde{h}\right\rangle$ can be transferred into a closed equation for $\langle\tilde{h}\rangle .2$ Details of calculations are presented in appendix A. This closed form reads

$$
\partial_{t}\langle\tilde{h}\rangle=\left(\hat{L}_{0}+C \hat{L}_{1}^{2}\right)\langle\tilde{h}\rangle
$$

The arising terms can be interpreted as renormalization of the coefficients in $\hat{L}_{0}$, i.e. the averaged evolution equation can be written

$$
\left\langle\partial_{t} h\right\rangle=\left\langle\left[\hat{L}_{0}+\delta \theta L_{1}\right] \tilde{h}\right\rangle=\hat{L}_{0}\left(w^{R}, a_{x}^{R}, a_{y}^{R}\right)\langle\tilde{h}\rangle
$$

with the renormalized coefficients

$$
\begin{gathered}
v_{0}^{\prime R}=v_{0}^{\prime} \\
\nu_{x}^{R}=\nu_{x}+C v_{0}^{\prime \prime 2} \\
\nu_{y}^{R}=\nu_{y} .
\end{gathered}
$$

Since $C$ is positive, the homogeneous noise always reduces the absolute value of $\nu_{\|}$, leading to weaker instability in the parallel modes (here we ignore the third and forth order derivatives form which may lead to further stabilizings or destabilizings). Thus, one may expect less pronounced pattern formation under this condition.

\subsection{Spatio-temporally fluctuating beams}

Renormalized coefficients of Eq.6.5 for the spatio-temporally fluctuation beams, case (iii), read

$$
\begin{gathered}
v_{0}^{\prime R}=v_{0}^{\prime}+\frac{C^{\prime \prime}}{2}\left(\nu_{x}^{\prime}+\nu_{y}^{\prime}\right) v_{0}^{\prime \prime} \\
\nu_{x}^{R}=\nu_{x}+\frac{C^{\prime \prime}}{2}\left(\nu_{x}^{\prime}+\nu_{y}^{\prime}\right) \nu_{x}^{\prime}+\frac{C}{2} v_{0}^{\prime \prime 2} \\
\nu_{y}^{R}=\nu_{y}+\frac{C^{\prime \prime}}{2}\left(\nu_{x}^{\prime}+\nu_{y}^{\prime}\right) \nu_{y}^{\prime}
\end{gathered}
$$

These renormalized coefficients are shown in Fig. 6.4 as functions of $\theta$. The functional dependence of the effective surface tensions on $\theta$ are completely changed by considering the noise effects. Thus, standard predictions of $\mathrm{BH}$

\footnotetext{
${ }^{2}$ For more details of Novikov's theorem see Novikov (1965).
} 

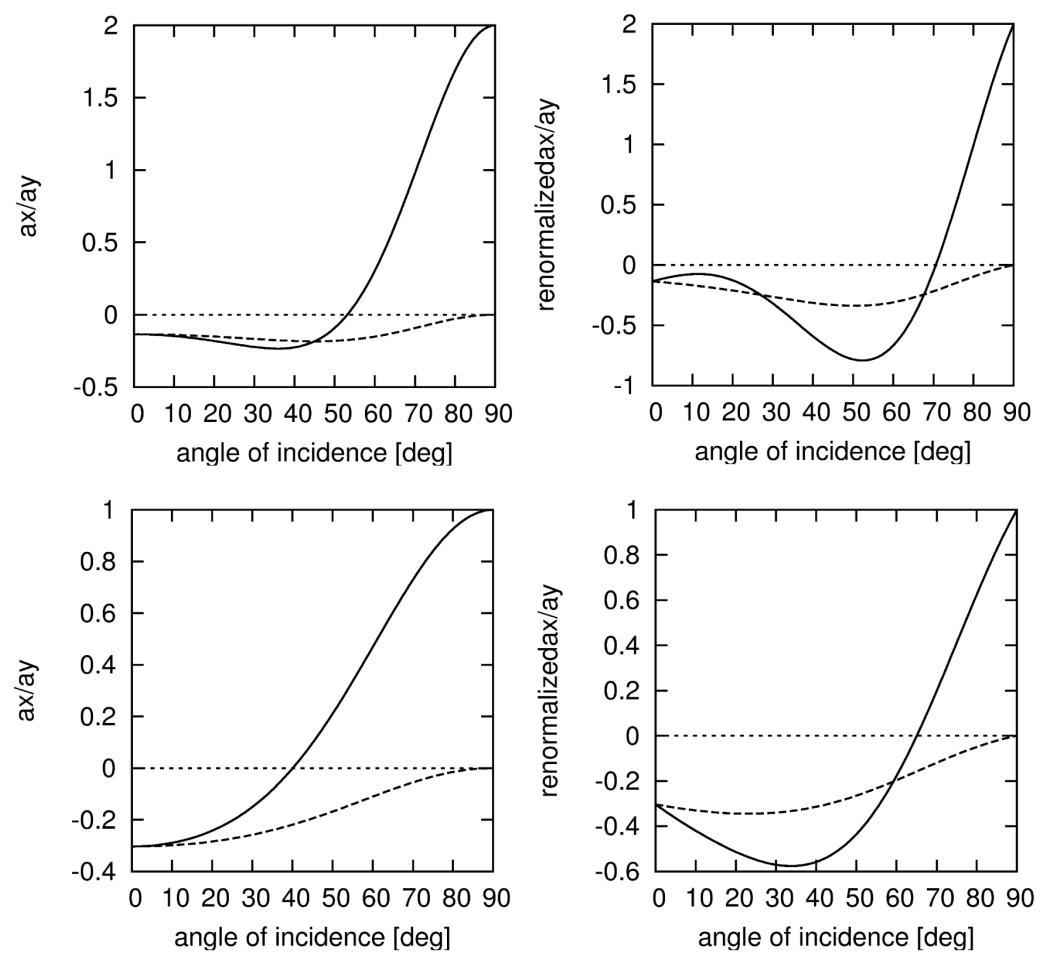

Figure 6.4: Renormalization of parameters $\nu_{x}\left(\theta_{0}\right)$ and $\nu_{y}\left(\theta_{0}\right)$ from Eq. 6.1 due to case (iii) beam profile noise. The left upper and lower panel show the behavior for an ideal beam. upper panel: $d / \sigma=d / \mu=1$, lower panel: $d / \sigma=d / \mu=2$, the right panels show the renormalization effects, if $\partial_{x}^{2} C(0)=\partial_{y}^{2} C(0)=0.2$. solid lines: $\nu_{x}$, dashed lines: $\nu_{y}$. The lower curve corresponds to faster growth within linear theory. Note that the dependence of the dominant ripple orientation for ideal beams are changed completely due to the noise.

model for orientation of patterns can be violated by beam fluctuations. Nevertheless, the experimental value of $C$ and its derivatives are not known and therefore a direct comparison to experiments is not possible.

We also performed KMC simulations with a broad beam of ions to observe generic effects of noise on pattern formation. Fig. 6.5 shows how surfaces under bombardment with beams profile (a) (see above) evolve. The main generic result is a slowing down in the process of pattern formation. Apart of that, the shape of morphologies and other features are not affected by the noise. In addition to this general situation, three specific cases of normal incidence angle, incidence near to the critical angle, and simulations with high diffusion 


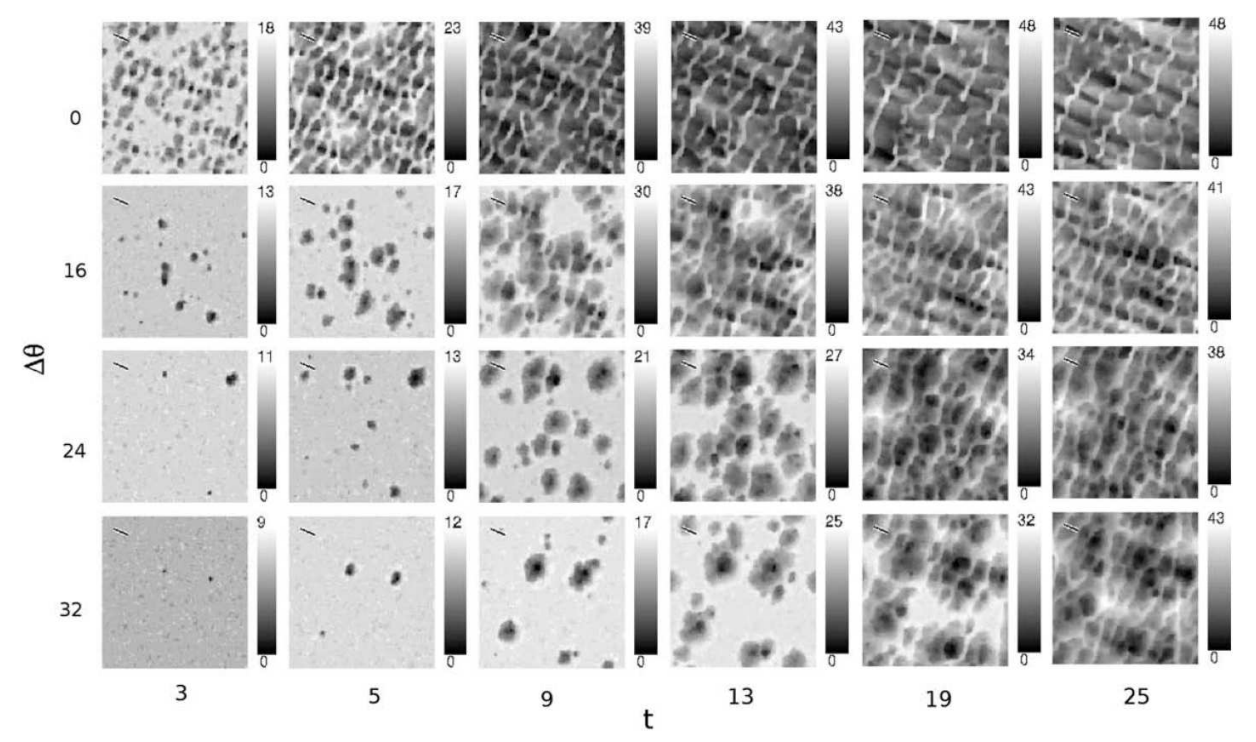

Figure 6.5: Time evolution of the surfaces which are sputtered by an ion-beam with flat distribution profile. Horizontal axis is time (ion/lateral atom) and vertical axis is the value of width $\Delta \theta$ in degrees. $\theta_{0}=50^{\circ}, L=256$. A significant delay induced by beam-noise is evident.

rate are examined.

\subsubsection{Normal incidence angle}

Fig. 6.6 compares the resulted structures in two cases of no divergence in the beam and a diverging beam with profile type (b). The corresponding PSD is shown in Fig. 6.7. Here, the rough patterns without any typical length scale (no pronounced peak in structure factor; green line) turn to patterns with some characteristic length scale (blue line). We performed the same simulations with rotating samples and as it is observed (red line) the same length scale is present in this situation as well.

\subsubsection{Grazing incidence angle}

Another situation, in which the width of beam profile may be crucial in pattern formation is sputtering at incidence angle close to the critical value $\theta_{c}$. As mentioned in section 3.4.1, increasing the incidence angle we reach a point where ripples rotate from direction perpendicular to the projection of the ion- 


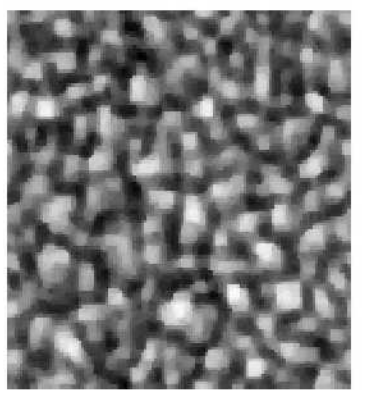

$0^{\circ}$
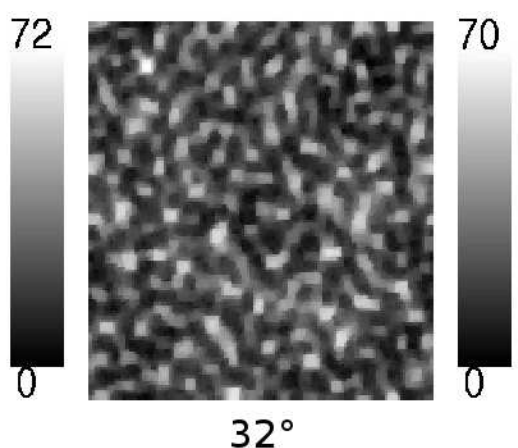

Figure 6.6: Noise induced effects in normal-incidence sputtering; Surface profiles sputtered by (a) an ideal beam (no noise) and (b) a beam with Gaussian profile $\Delta \theta=32^{\circ}$. By including noise in the beams, patterns with smaller length scales emerge.

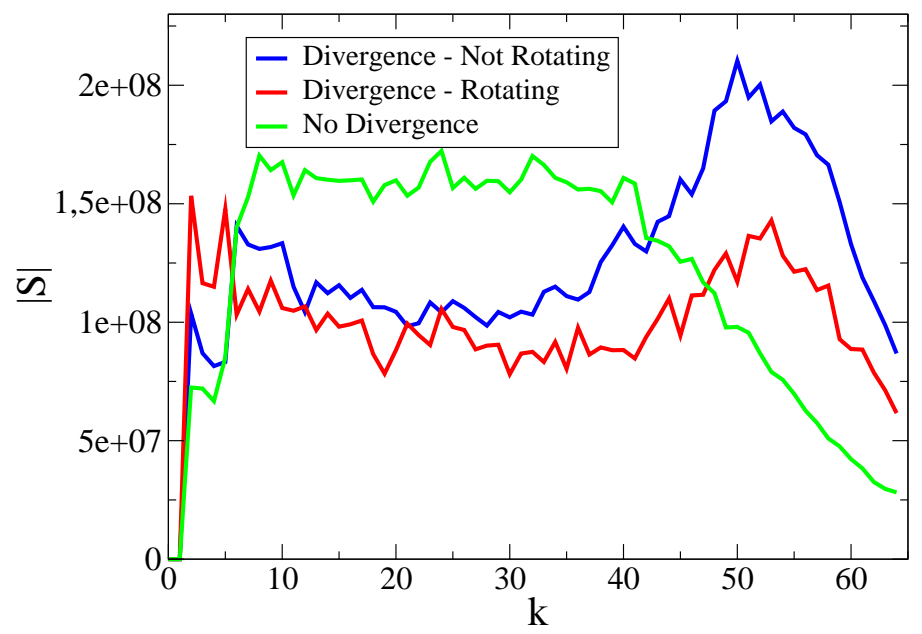

Figure 6.7: The modulus of structure factor of surfaces obtained from normal-incidence sputtering with non-zero ion-beam divergence (limiting cases with $\Delta \phi=0$ and $\Delta \phi=\pi$ ) compared to the case of sputtering by an ideal beam. Presence of beam-divergence leads to a length scale selection with the parameter set which does not produce mono-size structures with an ideal beam. 

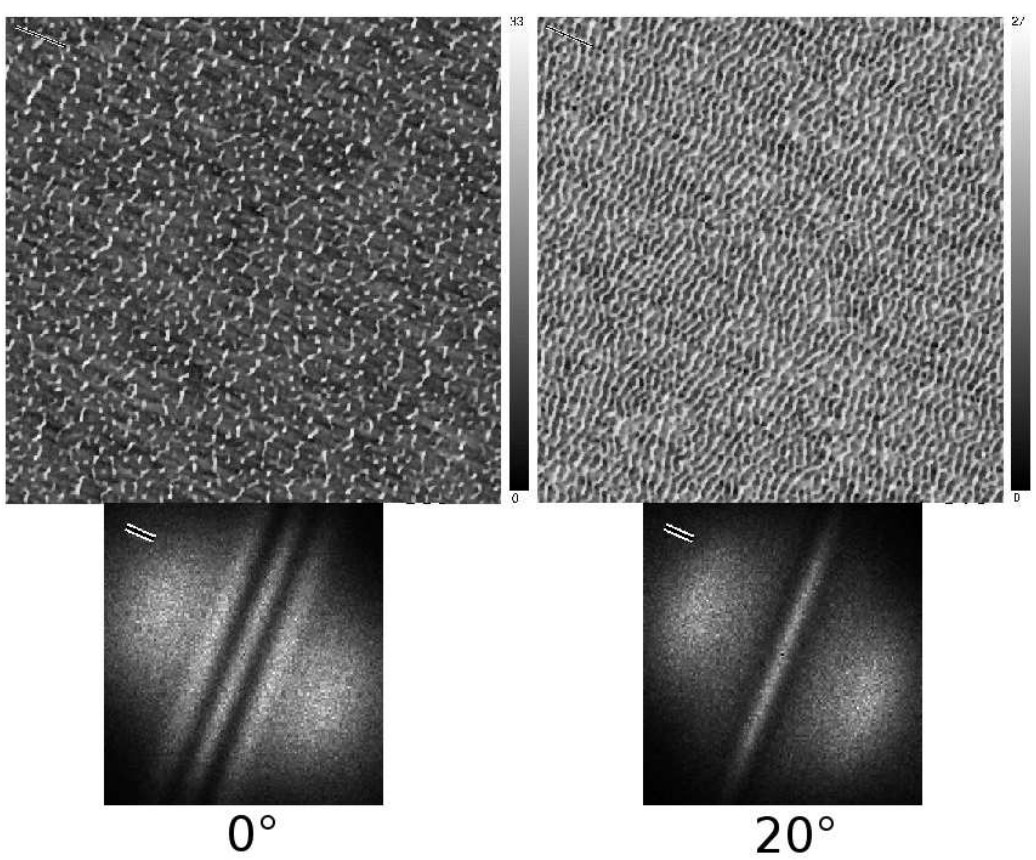

Figure 6.8: Simulation results for $\theta_{0}=65^{\circ}\left(\approx \theta_{\mathrm{c}}\right), \Delta \theta=0^{\circ}$ (left panel) and $20^{\circ}$ (right panel) at $\mathrm{t}=2$ ions/atom. Narrow bars indicate the azimuthal alignment of ion-beam. In lower panels corresponding surface profile Fourier transforms are shown. The regularity of parallel ripples enhanced by including the beam-noise.

beam to the parallel direction. A broad beam containing impinging ions with incidence angle slightly larger or smaller than $\theta_{0}$ may induce compound patterns of ripples in both directions. In Fig. 6.8, the effect of noise on patterns emerged by sputtering at $\theta=65^{\circ}$ is demonstrated. Surprisingly, ripples perpendicular to the ion-beam direction are longer and more regular in presence of beam-noise. The background structure parallel to the ion-beam direction is almost untouched by including the noise.

\subsubsection{High diffusion rate regime}

The last examined condition is sputtering with broad beam in high diffusion rate regime. We have chosen a partially enhanced diffusion attempt frequency $k_{1}=2.7 \cdot 10^{5}$ to bring the system to the edge of the region where EhrlichSchwoebel effects start to be dominant in pattern formation (see section 4.3.2). 

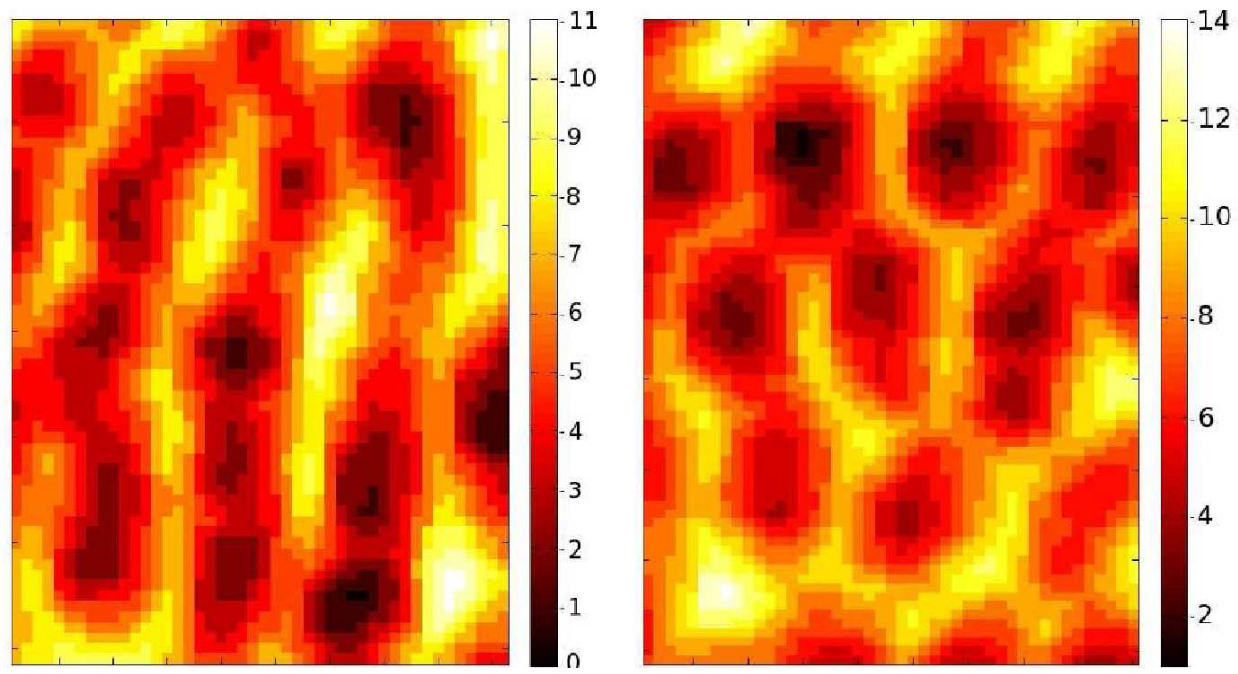

Figure 6.9: Simulation results of sputtering by an ion-beam directed along the $\mathrm{x}$-axis at an average angle of $\theta_{0}=50^{\circ}$ for $t=3$ ions/atom.Left panel:ideal beam, Right panel: Beam profile corresponding to the lowest panel of Fig. 6.2. The diffusion rate is enhanced by a factor of 300 in compare to default value. The presence of beam-noise favors the induction of Ehrlich-Schwobel effects.

We also took the beam profile (c) which is very close to what is present in experiments. In Fig. 6.9, changes in morphology induced by beam-noise is illustrated. The Ehrlich-Schwoebel effects (e.g. orienting regardless to beam direction) are enhanced by beam divergence. 


\section{Chapter 7}

\section{Rotating sample, dual-beam and sequential sputtering}

To produce a larger variety of structures and to improve their quality, more complex setups with multiple ion-beams, IBS on pre-structured samples, and rotating samples have been used, but few of them have been investigated in detail. In particular, Carter $(2004 ; 2005 ; 2006)$ has proposed the use of dual ionbeam sputtering (DIBS), Vogel and Linz (2007) proposed a four-beam setup and claimed that corresponding results may be obtained from a sequence of pre-structuring and stepwise beam or sample rotation using a single ion-beam (sequential ion-beam sputtering or SIBS). Continuous rotation of the sample or the ion-beam (referred to as RIBS, i.e. rotating IBS, in the following) has been proposed to suppress ripple formation by Zalar $(1985 ; 1986)$ (for example in SIMS and AES, where ripple formation would reduce the depth profiling resolution), but also to enhance the quality of isotropic structures (Bradley 1996). Recently, Joe et al. (2009) conducted a systematic experimental study of DIBS and SIBS on $\mathrm{Au}(001)$. They considered two different ion fluxes: a higher one, for which the orientation of patterns is determined by the ion-beam direction (erosive regime), and a lower one, for which surface diffusion becomes dominant in pattern formation process (diffusive regime). A number of their findings are not in accordance with expectations derived from the conventional continuum framework. This motivated us to study DIBS, SIBS and RIBS setups with Monte Carlo (MC) simulation methods. Recent proposals by Vogel and Linz (2007) and Muñoz-García et al. (2009) are based 
upon extensions of the standard continuum model (which is formulated as an anisotropic and noisy Kuramoto-Sivashinsky equation). These extensions have introduced new physical mechanisms, which change the scenario of pattern formation of the standard model, but none of these mechanisms have been confirmed and tested independently beyond doubt.

We present and discuss results obtained for the topographies, the shape and orientation of the ripples, the structure function and the evolution of roughness in different setups. We compare our findings to theoretical proposals, experimental results and to the standard linear continuum model.

\subsection{Simulation setup}

Throughout this work, we will focus on the erosion-dominated regime, leaving more complex interplays between erosion and diffusion in multi-beam and rotating setups for further studies.

Fig. 7.1 depicts the geometries of setups we use in the simulations. A DIBS setup consists of two ion-beam incidents from directions described by polar angles $\theta_{1}$ and $\theta_{2}$ and azimuthal angles $\phi_{1}$ and $\phi_{2}$ (see Fig. 7.1(a)). As a simple special case, we will consider opposing beams, i.e. $\theta_{1}=\theta_{2}$ and $\Delta \phi=\phi_{1}-\phi_{2}=180^{\circ}$. Crossed beams are studied for equal polar angles as well as for the general case of different polar and azimuthal angles.

SIBS setups are shown in Fig. 7.1(c). In a first step, ripples are produced by a single ion beam. Then we change the azimuthal angle of the ion-beam direction by some $\Delta \phi$ and monitor the further evolution of surface structures. Finally, Fig. 7.1(b) shows the RIBS setup, which is characterized by a constant angular velocity $\omega$ of the sample, which we realize by a fixed sample and the corresponding beam rotation. The evolution of structures and surface roughness will be systematically studied as a function of $\omega$.

The rotation of sample during IBS is equivalent to a fixed sample and a rotating ion-beam. Therefore rotation or any change of azimuthal angles in lab coordinates is simulated by keeping the surface fixed and rotating the ionbeam correspondingly. 


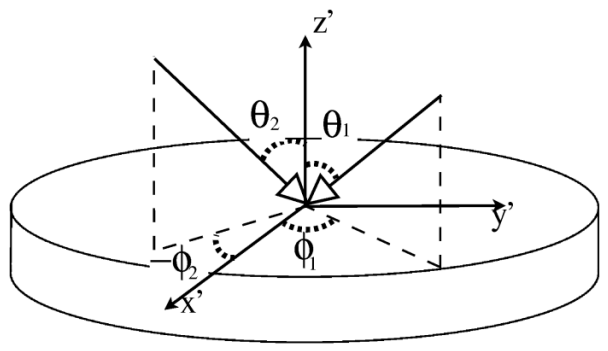

(a)

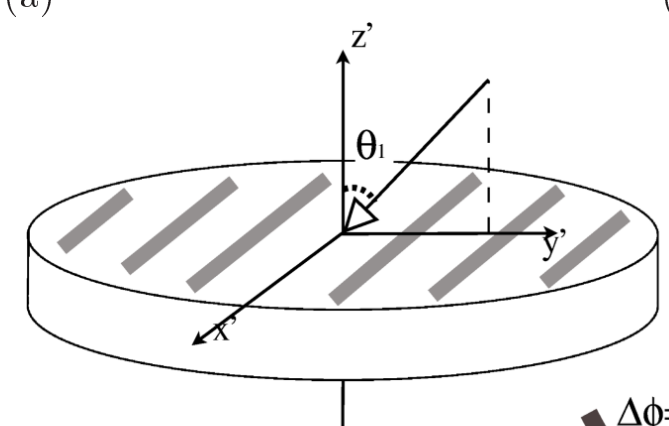

(b)
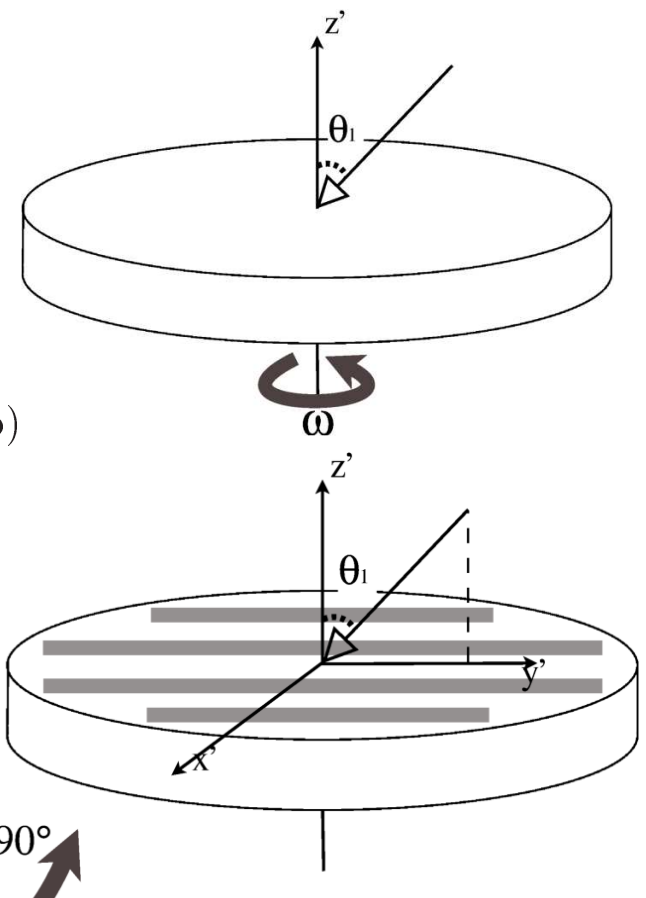

(c)

Figure 7.1: Three different IBS experiment setups. (a) dual ion-beam with fixed sample (DIBS). (b) continuously rotating sample with single ion-beam (RIBS). (c) sequentially sputtering of pre-rippled surfaces after $90^{\circ}$ rotation (SIBS). 


\subsection{Opposed ion-beams}

It has been proposed by Carter (2005) that IBS with two diametrically opposed ion-beams (same $\theta$ and a difference of $180^{\circ}$ in $\phi$ ) can lead to a cancellation of instabilities induced by each beam. Instead, he predicted temporal oscillations of ripple amplitudes. Furthermore, ripple motion should be suppressed due to the restoration of reflection symmetry, which would be broken by a single beam.

Fig. 7.2(b) shows results of topographies obtained from MC simulations of this setup. We never observe the predicted behavior, instead the growth of structures resembles that obtained in a single beam setup, as can be seen from comparing Figs. 7.2 (a) and (b). But ripples obtained from opposed beams appear longer and more straight than those from single beams and the patterns contain less defects. Furthermore, the shape of ripples changes significantly towards more symmetric slopes. In Fig. 7.3, we show a quantitative analysis of ripple slope angles $\alpha_{1}$ and $\alpha_{2}$, which are defined in the inset of the upper panel. To measure the symmetry of the slopes, we consider the asymmetry parameter

$$
\epsilon=\frac{\alpha_{2}-\alpha_{1}}{\alpha_{2}+\alpha_{1}}
$$

Histograms of this quantity are shown in Fig. 7.3 for single beam and opposed beam setups. The average value of $\epsilon$ is $\bar{\epsilon} \approx-0.08$ for single beams (indicating that the steeper slope is facing the beam), and $\bar{\epsilon} \approx 0.01$ for opposed beams. We also checked that the skewness of the $\epsilon$-distribution is reduced by using opposed beams. In conclusion, opposed ion-beams do not lead to a cancellation of instabilities, but help to produce more symmetric ripples and patterns with less defects as compared to a single ion-beam setup.

\subsection{Crossed ion-beams}

Setups of multiple ion-beam incidents from different directions have been proposed by Carter (2004;2005). Vogel and Linz (2007) developed a continuum theory for a setup with four beams all with the same polar angles of incidence and crossing with $\Delta \phi=90^{\circ}$. Their theory is based on a damped KuramotoSivashinsky equation and takes into account terms up to fourth order in a 
(a)

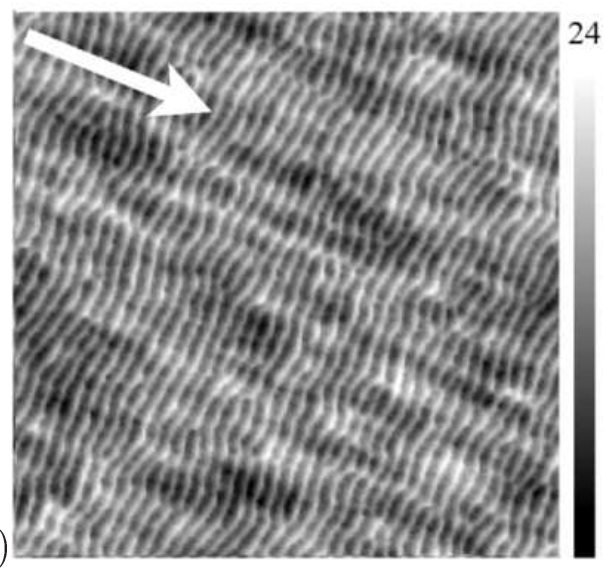

24

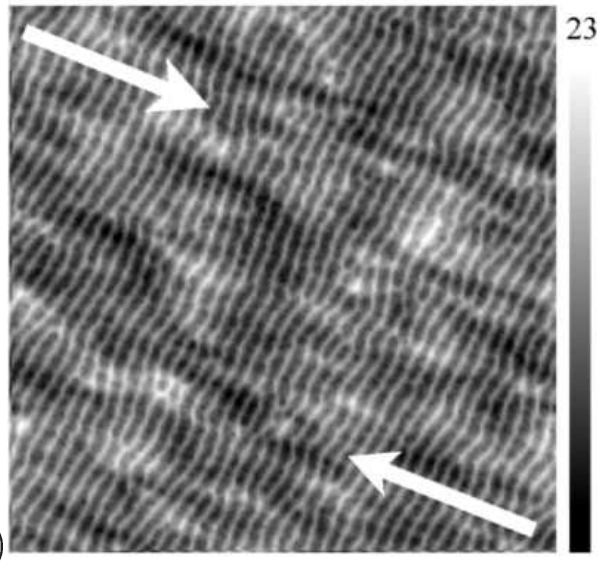

Figure 7.2: Rippled surfaces after 8 ions/atom of sputtering. (a) by a single ion-beam, (b) by two ion-beams opposed to each other. Arrows indicate the direction of ion-beams.

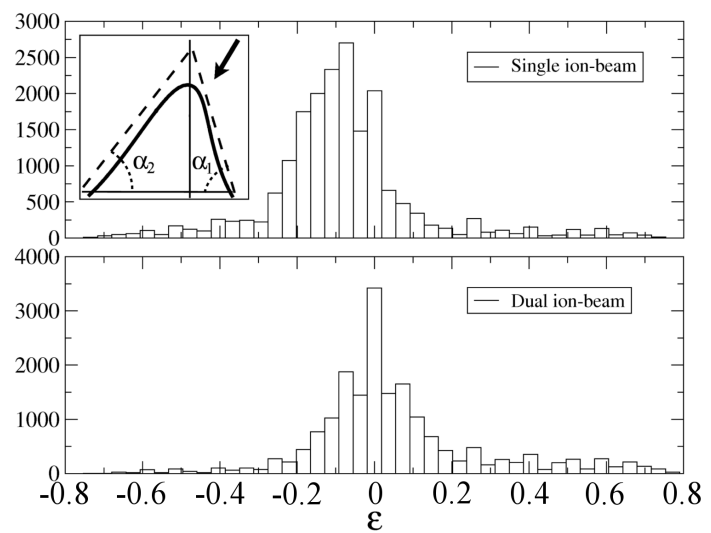

Figure 7.3: Distribution function of the asymmetry parameter $\epsilon$ (see main text) for the cases of a single ion beam (upper panel) and two ion-beams opposed to each other (lower panel). 
gradient expansion of the erosion term. They predict square patterns, which can be stable in the long time regime under specific conditions. Joe et al. (2008) performed experiments with two ion-beams with $\theta=73^{\circ}$ and $\Delta \phi=90^{\circ}$ on $\mathrm{Au}(001)$. In the erosive regime, they find nano-dot patterns with square symmetry (albeit with a rather low degree of order), if the fluxes of the two beams are precisely balanced. Otherwise, the structures develop into modulated ripples.

In the upper row of Fig. 7.4, we show simulation results for perfectly balanced ion-beams (beam (1) in Table 7.1). The middle row of Fig. 7.4 shows results for ion-beams with different intensities incident from the same polar angle (beams (2) and (3) in Table 7.1) and the lower row shows corresponding results for ion-beams of identical intensities, but incident from different polar angles and with different beam parameters (beams (1) and (4) in Table 7.1). In Table 17.1, $\nu_{\|}$and $\nu_{\perp}$ denote the effective surface tension coefficients (parallel and perpendicular to the beam projection onto the $x-y$ plane), which appear as prefactors of the second spatial derivatives of the height field in linear Bradley-Harper (BH) theory. They determine the topography dependent part of the erosion rate within linear BH theory for long wavelength (Bradley and Harper 1988).

White arrows in Fig. 7.4 indicate the projection of the ion-beams into the $x-y$ plane (thus they enclose $\Delta \phi$ ). The geometry is chosen such that the $\mathrm{x}$-axis always is the bisector of this angle. For balanced beams and $0 \leq \Delta \phi<90^{\circ}$, we observe ripples with a wavevector parallel to the x-axis, for $90^{\circ}<\Delta \phi<180^{\circ}$, the ripple wavevector is parallel to the y-axis, but the ripple pattern shows more defects. Exactly at $\Delta \phi=90^{\circ}$, square patterns replace the ripples. For unbalanced beams, we observe ripples in oblique directions, which we will discuss below.

The middle and lower row of Fig. 7.4 are given to emphasize that we did not observe any differences in pattern formation due to different mechanisms of imbalance. The middle row corresponds to beams of different intensities, characterized by the ratio $f=J_{<} / J_{>}$of the smaller ion flux $J_{<}$and the larger flux $J_{>}$(here $f=1 / 2$ ). The ion flux multiplies all terms in the gradient expansion of the erosion rate. Other mechanisms to produce imbalanced erosion rates, which we tried, lead to the same patterns, if they imply the same erosion 
rates of linear theory.

Note that in our simulations both $\nu_{\|}$and $\nu_{\perp}$ are negative, indicating that erosion leads to growing height fluctuations in both directions within the linear BH theory. The linear theory of DIBS as outlined by Joe et al. (2009) indicates the appearance of growing crossed ripples for this situation, in accordance with our simulation results. In the experiments by Joe et al. (2009), however, the system corresponds to $\nu_{\|}=14.2$ and $\nu_{\perp}=-0.9$. This set of effective surface tensions would result in a stable flat surface, in contradiction to the experimental observations.

Let us analyze our findings within the simple framework of linear BH theory, for which contributions of both beams to the erosion rate are simply added. Suppose beams 1 and 2 are characterized by effective surface tension coefficients $\nu_{A}^{(b)}$ with $b$ being 1 or 2 and $A$ being $\|$ and $\perp$. Averages of these coefficients over the two beams are denoted by $\bar{\nu}_{A}=\left(\nu_{A}^{(1)}+\nu_{A}^{(2)}\right) / 2$ and we introduce $\Delta \nu_{A}=\left(\nu_{A}^{(1)}-\nu_{A}^{(2)}\right)$. According to linear theory, the growth of Fourier modes $\left|h\left(k_{x}, k_{y}, t\right)\right| \propto \exp (\Gamma t)$ due to erosion is controlled by the (real) growth rate $\Gamma\left(k_{x}, k_{y}\right)$, which is a quadratic form of the wavevector, i.e. $\Gamma=\mathbf{k}^{t} \hat{\nu} \mathbf{k}+O\left(k^{4}\right)$ with a matrix $\hat{\nu}$ of effective surface tensions. In the presence of isotropic surface diffusion, the direction of the fastest growing mode is obtained by determining the largest eigenvalue of $\hat{\nu}$ and the corresponding eigenvector. $\hat{\nu}$ is easily calculated. Its matrix elements take on the form

$$
\begin{aligned}
\hat{\nu}_{x x} & =2\left(\bar{\nu}_{\|}+\bar{\nu}_{\perp}\right)+\left(\bar{\nu}_{\|}-\bar{\nu}_{\perp}\right) \cos \Delta \phi \\
\hat{\nu}_{y y} & =2\left(\bar{\nu}_{\|}+\bar{\nu}_{\perp}\right)-\left(\bar{\nu}_{\|}-\bar{\nu}_{\perp}\right) \cos \Delta \phi \\
\hat{\nu}_{x y} & =\left(\overline{\Delta \nu}_{\|}-\overline{\Delta \nu}_{\perp}\right) \sin \Delta \phi
\end{aligned}
$$

and $\hat{\nu}_{y x}=\hat{\nu}_{x y}$. For balanced beams, $\Delta \nu_{A}$ vanishes and the matrix of surface tension coefficients becomes diagonal, indicating that ripples will only appear with wavevectors either parallel to the $\mathrm{x}$-axis or parallel to the $\mathrm{y}$-axis. At $\Delta \phi=90^{\circ}$, the rates in both directions become degenerate and square patterns will emerge, if they are stabilized by the nonlinear terms. As $\cos (\Delta \phi+\pi / 2)=$ $-\cos \Delta \phi$, it is obvious from Eq. 7.2 that the regime $90^{\circ}<\Delta \phi<180^{\circ}$ can be mapped to $0<\Delta \phi<90^{\circ}$ by interchanging $\mathrm{x}$ and $\mathrm{y}$. This explains the main features of the upper row of Fig. 7.4.

For unbalanced beams, the orientation of ripples will generally depend both 
Table 7.1: Beam parameters for the DIBS setup

\begin{tabular}{ccccccc} 
& $\theta$ & $a$ & $\sigma$ & $\mu$ & $\nu_{\|}$ & $\nu_{\perp}$ \\
\hline$(1)$ & $50^{\circ}$ & 9.3 & 3.0 & 1.5 & -4.4 & -0.86 \\
$(2)$ & $50^{\circ}$ & 9.3 & 3.0 & 1.5 & -5.8 & -1.1 \\
$(3)$ & $50^{\circ}$ & 9.3 & 3.0 & 1.5 & -2.9 & -0.57 \\
$(4)$ & $35^{\circ}$ & 7.3 & 3.0 & 1.5 & -1.5 & -0.81 \\
$(5)$ & $50^{\circ}$ & 3.8 & 2.2 & 1.5 & -0.67 & -0.53
\end{tabular}

on $\Delta \phi$ and the imbalances in surface tension coefficients $\Delta \nu_{\|}, \Delta \nu_{\perp}$, but for $\Delta \phi=90^{\circ}$ the situation is simpler. The cosine terms vanish, and the eigenvectors $\mathbf{e}_{ \pm} \propto(1, \pm 1)$ of the simpler matrix become independent of the rate imbalances. Thus, the linear theory predicts ripples with wavevectors inclined by $\psi=45^{\circ}$ or $\psi=135^{\circ}$ with respect to the $\mathrm{x}$-axis, i.e. parallel to one of the beams (from the eigenvalues it follows that the wavevector is parallel to the dominant beam), irrespective of the amount or nature of the imbalance in growth rates, if $\Delta \phi=90^{\circ}$. This result is in accordance with the experimental findings by Joe et al. (2009) as well as our simulations (see Fig. 7.4 (e) and (h)). The directions $\psi$ for other values of $\Delta \phi$ are easily obtained, if the imbalance is known. In Fig. 7.4, we have indicated these directions with dashed white lines. We find a satisfactory agreement of our simulation results with this prediction. Fig. 7.5 depicts the predicted deviations of the orientation of ripple wavevectors from the $\mathrm{x}$-axis (i.e. the bisector of the beam directions) as a function of $\Delta \phi$ for different ratios $f$ of fluxes of beams with the same beam parameters and the same angle of incidence. $f=1$ corresponds to balanced beams, whereas $f=0$ corresponds to a single ion-beam. Values of $f$ between 0 and 1 smear out the step of the $f=1$ and lead to a family of sigmoid curves interpolating smoothly between the limiting cases.

In conclusion, our simulations show crossed ripple patterns for balanced, orthogonal beams as observed by Joe et al. (2009). For non-balanced or nonorthogonal beams, simple ripple patterns appear in directions, which are in accordance with linear continuum theory. These directions are not changed at longer times, when the growth of the ripple amplitudes has saturated due to nonlinearities. 


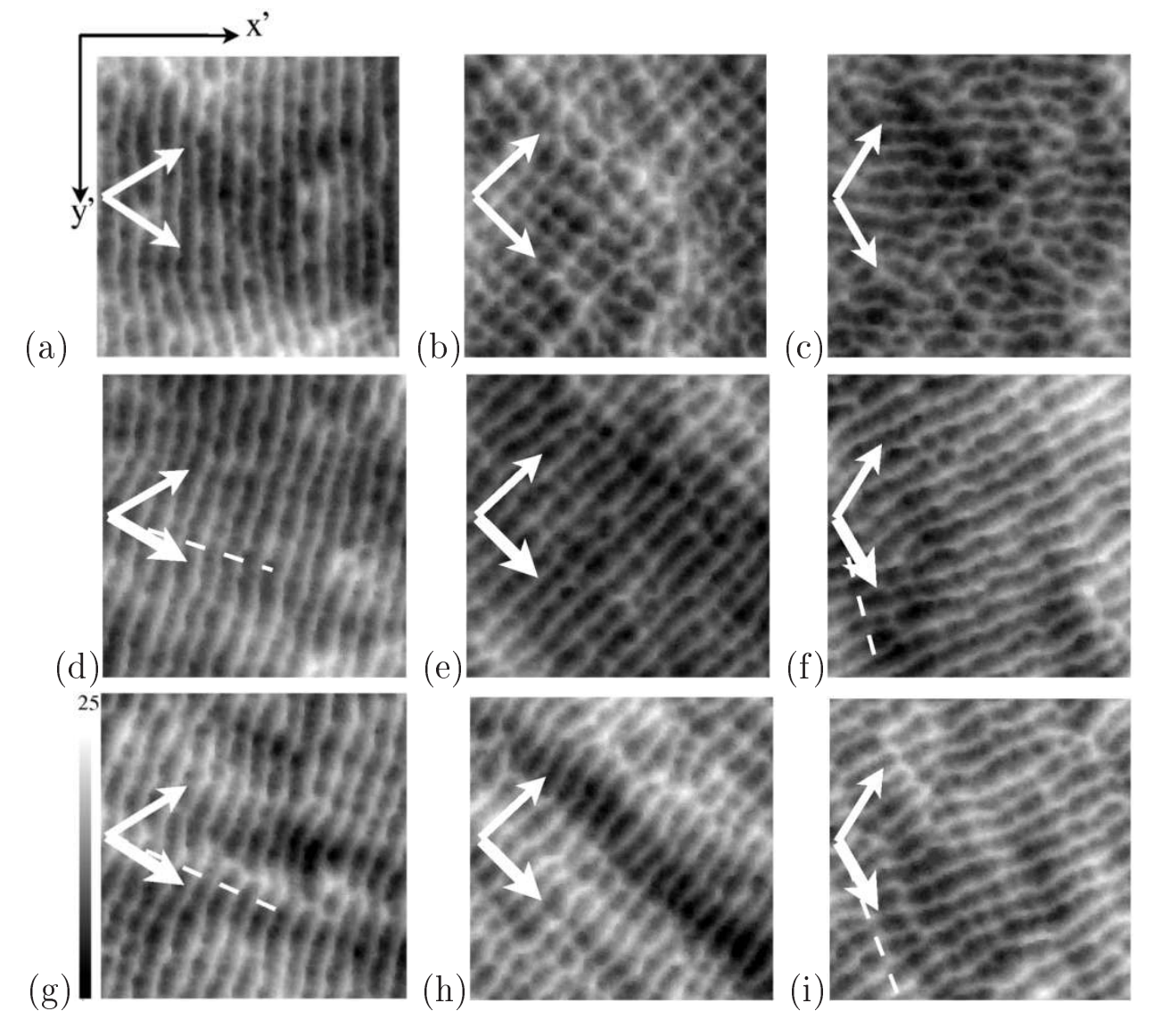

Figure 7.4: upper row: Rippled surfaces after 40 ions/atom sputtering by two balanced ion-beams incident from $\theta=50^{\circ}$ and separated in azimuthal angle by (a) $\Delta \phi=60^{\circ}$, (b) $\Delta \phi=90^{\circ}$ and (c) $\Delta \phi=120^{\circ}$. middle row: same as (a)-(c) but for imbalanced ion-beams, which differ in intensity by a factor of $2(f=1 / 2)$ corresponding to beams (2) and (3) in Table 7.1 lower row: same as middle row, here the imbalance is generated by different incident angles and beam parameters, (1) and (4) in Table 7.1. Arrows indicate the directions of ion-beams. In middle and lower row bigger arrows correspond to the dominant ion-beam. Dashed lines indicate the directions predicted for the wavevectors of ripples by linear theory (see main text). 


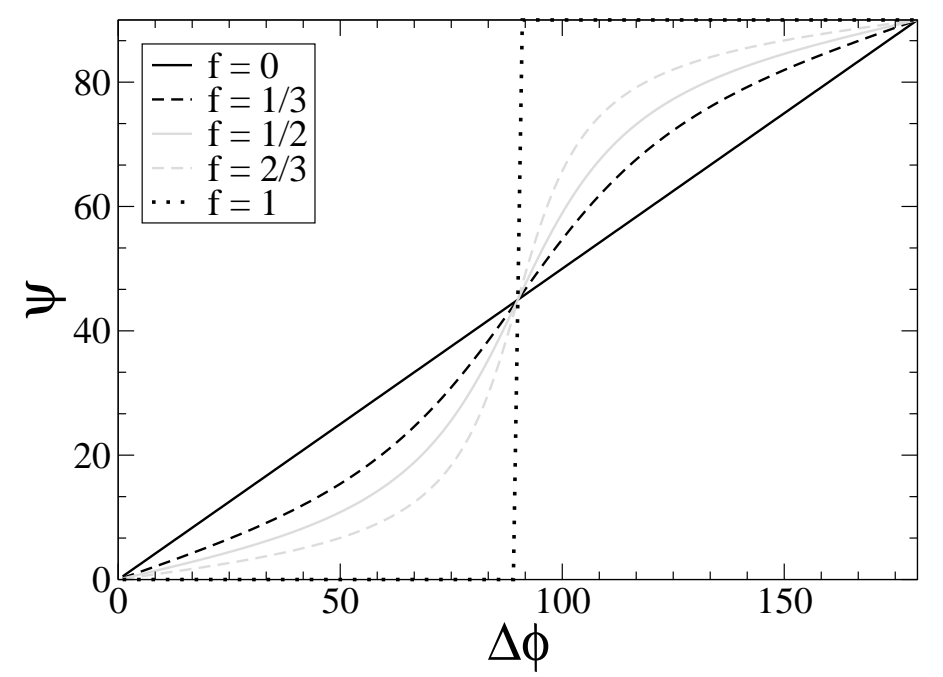

Figure 7.5: Orientation of fastest growing mode for different values of $\Delta \phi . \psi$ denotes the angle of the ripple wavevector with the x-axis. Different lines correspond to different ratios of ion fluxes $f$ (see main text).

\subsection{Sputtering of rippled surfaces}

Since the possibilities of simultaneous sputtering by multiple beams are often limited in experiments, sequential sputtering by a single beam from different directions seems to be a more promising setup. Vogel and Linz proposed SIBS as a general substitute for multi-beam sputtering (Vogel and Linz 2007). Note that in SIBS setups, a precise balance between fluxes of multiple beams, which may be difficult to achieve in DIBS, can be adjusted by tuning the exposure time in each direction.

Joe et al. (2009) performed experiments on $\mathrm{Au}(001)$ using an ion-beam incident from $\theta=72^{\circ}$. In the erosive regime, ripples with wavevectors perpendicular to the projection of the beam direction into the initial surface plane ( $k_{\perp}$ modes) build up initially. After rotating the target by $90^{\circ}$ (keeping $\theta$ fixed), they observe that the ripple pre-structure is very rapidly destroyed and new ripples build up in the correspondingly rotated direction. But the authors could never observe patterns corresponding to a superposition of two generations of ripples. 
We have simulated SIBS with ion-beams incident from $\theta=50^{\circ}$ and a rotation step of $\Delta \phi=90^{\circ}$ after 9 monolayers of erosion. Our parameters are chosen such that $k_{\|}$-mode ripples (i.e. wavevector parallel to the beam direction on the surface) appear initially (see Fig. 7.6(a)). After the rotation step, a correspondingly rotated ripple pattern builds up as shown in Fig. 7.6(c). In contrast to the experiment, we could observe a superposition of ripples of both orientations in a narrow time window, shortly after the rotation step (shown in Fig. 7.6(b), at $t=10.8$ ions/atom). The degree of order of these superimposed patterns can be seen from the structure factor in Fig. 7.6(d).

Let us discuss the surface evolution following a rotation step. From linear $\mathrm{BH}$ theory we expect every Fourier mode $h\left(k_{\|}, k_{\perp}\right)$ to grow or decay exponentially with a rate $\Gamma\left(k_{\|}, k_{\perp}\right)=-\nu_{\|} k_{\|}^{2}-\nu_{\perp} k_{\perp}^{2}-B\left(k_{\|}^{2}+k_{\perp}^{2}\right)^{2}$. Here, we have explicitly included an isotropic surface diffusion term proportional to $B$ as a smoothing mechanism. Immediately before a rotation step, a rippled pattern has developed, which consists of Fourier modes, centered around the fastest growing mode. Let us assume that this is a $k_{\|}$mode (corresponding to our simulations). Then its wavevector is $\left(k_{\|}, k_{\perp}\right)=\left(k_{m}, 0\right)$ with $k_{m}=\left|\nu_{\|}\right| /(2 B)$. A $90^{\circ}$ rotation step transforms this mode into $\left(0, k_{m}\right)$, and the subsequent evolution starts with a correspondingly rotated pre-structure. The growth rate $\Gamma\left(0, k_{m}\right)$ of the dominant mode of the pre-structure, measured in units of the maximal growth rate $\Gamma\left(k_{m}, 0\right)=\nu_{\|}^{2} /(4 B)$, is given by $r=\Gamma\left(0, k_{m}\right) / \Gamma\left(k_{m}, 0\right)=-\left(1+2 \nu_{\perp} /\left|\nu_{\|}\right|\right)$. Note that it is independent of $B$. From this, we conclude that linear theory predicts the following features of the evolution of patterns: (i) if $\nu_{\perp}>0$ the rate $r<-1$, which implies that the decay of the pre-structure (at least the mode $\left.\left(0, k_{m}\right)\right)$ appears faster than the growth of the fastest mode, i.e. $\left|\Gamma\left(0, k_{m}\right)\right|>\Gamma\left(k_{m}, 0\right)$, (ii) if $\nu_{\perp}<0$ but $\left|\nu_{\perp}\right|<\left|\nu_{\|}\right| / 2$ then $-1<r<0$ so that the decay of the $\left(0, k_{m}\right)$ mode is slower than the growth of the fastest mode and (iii) if $\nu_{\perp}$ is negative and $\left|\nu_{\perp}\right|>\left|\nu_{\|}\right| / 2$ then $r>0$, i.e. linear theory predicts that the pre-structured $\left(0, k_{m}\right)$ mode will grow. A completely analogous discussion holds, when the initially formed ripples are made of $k_{\perp}$ modes, i.e. when $\nu_{\perp}<0$ and $\nu_{\perp}<\nu_{\|}$. We just have to interchange $\|$and $\perp$ in the above expressions. This is the situation which is realized in the experiment by Joe et al. (2009). Furthermore, let us remark that the gradient expansions presented by Makeev et al. (2002) always produces $\nu_{\perp}<0$, so that case (i) 
of the above discussion will not show up, but it may be realized, if the initial ripples are $k_{\perp}$ modes.

For the parameters of our simulation $\left(\nu_{\perp}=-0.86\right.$ and $\left.\nu_{\|}=-4.4\right)$ the prestructure should decay with $r \approx-0.81$, i.e. slower than the growth of the new ripples. This is roughly in accordance with our simulations, albeit only after a latency of $\approx 1 \mathrm{ion} /$ atom during which the amplitude of the old pattern stays constant ( see the upper inset of Fig. 7.7). From our data of the decay and the growth after this latency we estimate $r \approx-0.80$. This only leaves a time window of less than 1 ion/atom (crossover appears at $\approx 0.72$ ions/atom), during which a superposition of the pre-structure and the new structure can be observed. For the experiments by Joe et al. (2009) $r \approx-17.8$, i.e. the pre-structure decays extremely fast compared to the growth of new ripples, and the corresponding time window of coexisting new and old ripples is much smaller, which may explain why no superposition of ripple patterns has been observed.

The rapid decay of the finite amplitude pre-structure leads to a transient decrease of roughness. We define a structural relaxation time $T_{0}$ as the length of the interval from the rotation step through the transient decrease of roughness to the point, where roughness has retained its value immediately before the rotation step. This time interval contains all processes necessary to rotate the ripple pattern from the previous to the current rotation step. In our case $T_{0} \simeq 2.5 \mathrm{ion} /$ atom. We expect this timescale also to be a relevant crossover scale for rotating beams or samples, which will indeed be confirmed in the next section.

We also tried to test the prediction (iii), i.e. a growth of the pre-structure if $\left|\nu_{\perp}\right|>\left|\nu_{\|}\right| / 2$. To this end we performed SIBS simulations with beam 5 of Table 7.1, i.e $\nu_{\|}=-0.67$ and $\nu_{\perp}=-0.53$. Results are shown in the lower inset of Fig. 7.7. As $\nu_{\|}$and $\nu_{\perp}$ approach each other, the ripples becomes more and more blurred, so that it makes no sense to try to identify crossed ripple structures. But the pronounced dip in the roughness has vanished, indicating that there is at least no decay of any dominant, finite amplitude part of the spectrum of $\left(k_{\|}, k_{\perp}\right)$ modes. However, we could not identify any growing modes within the spectrum of the pre-structure. 
(a)

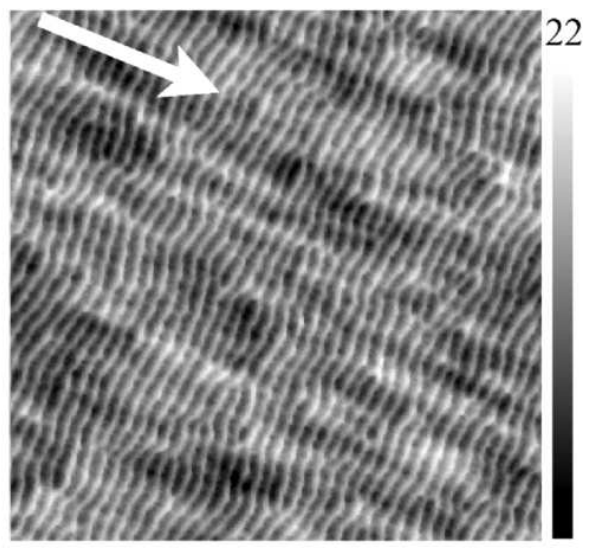

(c)

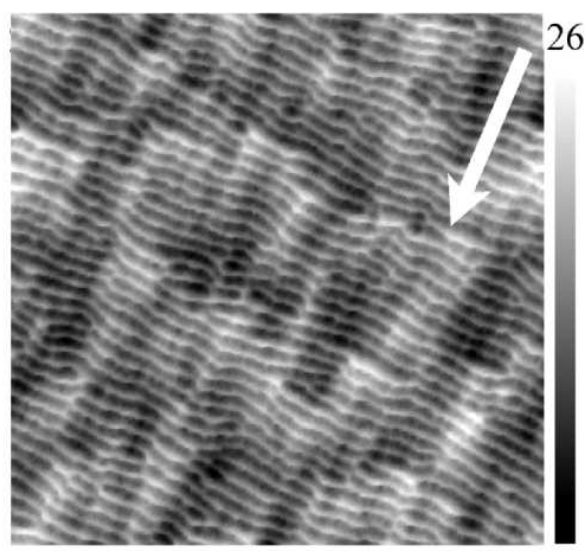

(b)

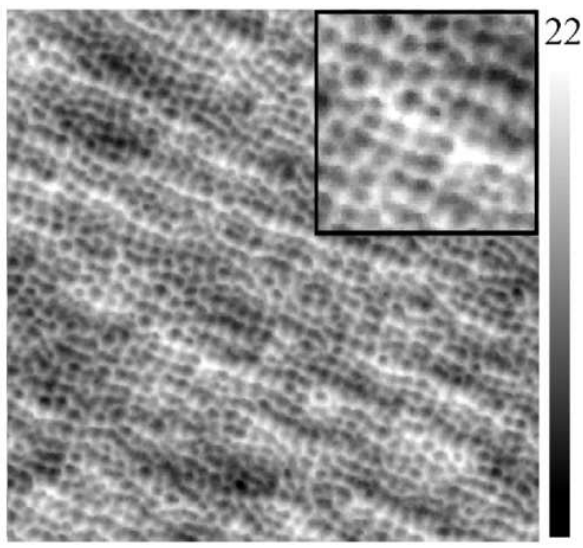

(d)

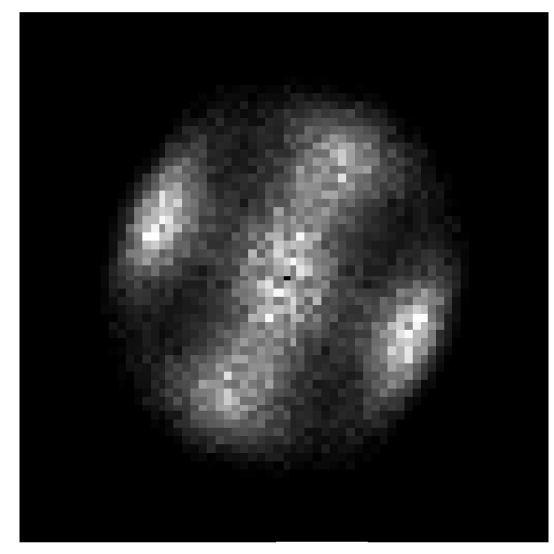

Figure 7.6: Snapshots of surface profile during SIBS. At $t=9$ ions/atom, a $\Delta \phi=90^{\circ}$ rotation step occurs. Shots are at (a) $t=9$ ions/atom, (b) $t=10.8$ ions/atom (inset: zoomed in) and (c) $t=18$ ions/atom. Arrows indicate the direction of ion-beams. In panel (d) the structure factor $\left|h\left(k_{x}, k_{y}\right)\right|^{2}$ of the profile of panel (b) is shown. 


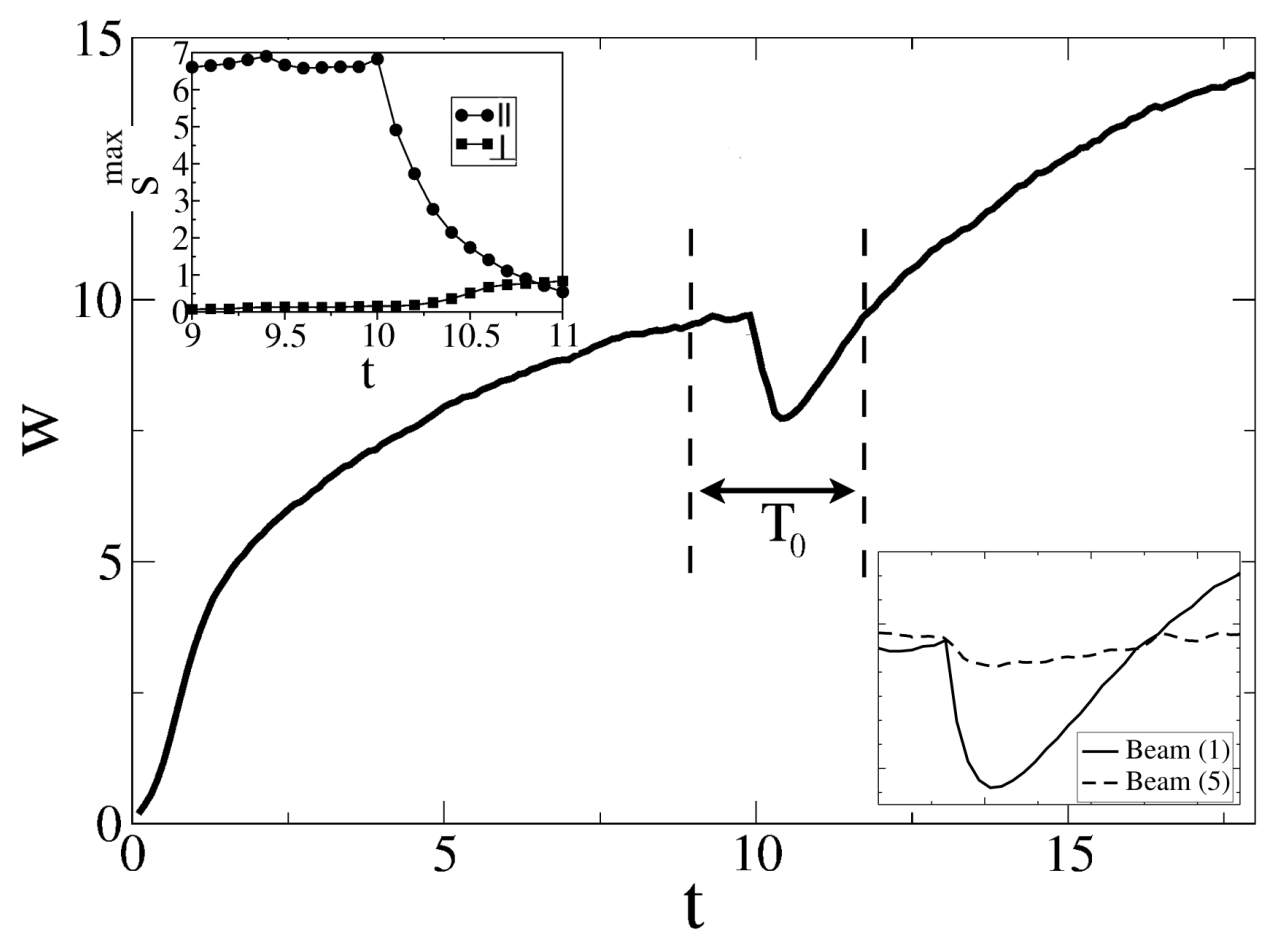

Figure 7.7: Roughness evolution of surface patterned by SIBS. The surface is rotated by $90^{\circ}$ at $t=9$ ions/atom. The upper inset shows the temporal evolution of the maximum of the structure factor $S_{\max }=\max \left(|h(\vec{k})|^{2}\right)$ for wavevectors in $\|$ and in $\perp$ direction. The lower inset shows that there is no dip in roughness in the regime of surface tension coefficients, for which linear $\mathrm{BH}$ theory would predict a growth of the pre-structure $\left(\nu_{\|}=-0.67, \nu_{\perp}=-0.53\right)$ 


\subsection{Sputtering of continuously rotating sample}

Sample rotation during IBS is applied for various reasons. One motivation is to achieve suppression of pattern formation in SIMS and AES depth profiling (Zalar 1985;1986). There are numerous reports that RIBS can suppress surface roughening and enhance the resolution of depth profiling (see Carter (1998) and references therein). Although this method is frequently used, there are very few systematic studies of the effects of different parameters, in particular of the angular velocity of rotation (Tanemura et al. 1992). Previous discrete simulations could not successfully explain the observed strong suppression of roughening (Koponen et al. 1997). Recently, IBS has become a popular method for smooth etching of metallic surfaces (Reichel et al. 2007). Here too, sample rotation has been proposed as a practical measure (Reichel et al. 2007) to prevent nano-scale roughening. A different motivation to use RIBS is the control of pattern formation. As mentioned in chapter 2, Frost et al. have found that off-normal IBS with sample rotation may lead to formation of hexagonal, close packed dot patterns. This is attributed to a restoration of axial rotation symmetry with respect to the average surface normal, which is broken by off-normal incidence of a single ion-beam at fixed azimuthal angle. Dot formation in rotated, off-normal IBS has been found in the framework of continuum theories (Bradley 1996, Frost 2002; Castro et al. 2005), assuming a flux of incoming ions, which is distributed evenly over all azimuthal angles. This assumption corresponds to the limit of high rotation frequencies. Dots also appear in MC simulations performed in the high rotation frequency limit for a wide range of parameters (Yewande et al. 2007).

In the present work, we focus on the systematic dependence of height fluctuations on the rotation frequency, irrespective of the random or deterministic nature of these fluctuations. Therefore, we will study the roughness $w=\left\langle(h-\langle h\rangle)^{2}\right\rangle$, averaged over the sample and an ensemble of 40 realizations, as function of time and rotation frequency $\omega$. Reported rotation frequencies cover a range from 0.1 to $15 \mathrm{rpm}$ for different fluxes and different types of ions and materials (Frost and Rauschenbach 2003; Zalar 1985, Zalar 1986, Konarski and Hautala 1995, Cui et al. 2005). There is a predictions of the scaling of height with $\omega$, which, in Ref. Bradley and Cirlin (1996), is given in 
the form

$$
h(\vec{k}, t) \propto \exp \left(\frac{\left(\nu_{\perp}-\nu_{\|}\right)}{4 \omega} k^{2} \sin (2 \omega t)\right) .
$$

It is based on the original linear Bradley-Harper theory in rotating coordinate systems. Furthermore, Cui et al. (2005) reported that ripples do not form for angular frequencies greater than $0.1 \mathrm{rpm}$ (for a flux of $3.5 \times 10^{14} \mathrm{ions} \mathrm{cm}^{-2} \mathrm{~s}^{-1}$ of $300 \mathrm{eV} \mathrm{Ar}^{+}$on GaN substrate).

In section 7.4, we have defined a characteristic response time $T_{0}(\Delta \phi)$ of the IBS generated structures to sudden changes $\Delta \phi$ of the beam direction. We propose that this time scale is also of relevance for RIBS, as surface structures might follow rotation frequencies much smaller than $\omega_{0}=\Delta \phi / T_{0}$ adiabatically. On the other hand $\omega \gg \omega_{0}$ might correspond to the high frequency limit. We performed simulations with different rotation frequencies varying in the range $0.05 \cdots 50 \omega_{0}$, with $\omega_{0} \simeq 36^{\circ}$ per eroded mono-layer taken from our SIBS simulations. For low frequencies, ripples form and rotate in synchrony with the beam direction (see Fig. 7.8(d-f)). For high frequencies, $(\omega=\infty$ is included as it corresponds to random azimuthal directions of incoming ions chosen from a flat distribution), cellular structures of growing size are observed (Fig. 7.8(c)). These findings are in agreement with predictions by Bradley (1996). A mixture of short ripples and cellular structures appears at intermediate $\omega$ (Fig. 7.8(b)). Somewhat surprisingly, the roughness of the surface is not a monotonous function of rotation frequency. In Fig. 7.9 roughness is shown as a function of time for different $\omega$. For low (including $\omega=0$ ) and high frequencies, it grows monotonically, approaching approximately equal growth rates beyond $t \approx 30$, independent of $\omega$. For intermediate $\omega$, the roughness shows oscillations (with frequencies $\Omega \approx 4 \omega$ ), and its growth rate is strongly suppressed. For $\omega=3.5 \omega_{0}$ growth rate reaches a minimum. In Fig. 7.10 the roughness, averaged over a period $T=2 \pi / \omega$, is shown against $\omega$, for times up to $t=37 \mathrm{ions} /$ atom . For increasing $t$, the minimum at $\omega \simeq 3.5 \omega_{0}$ becomes more and more pronounced. Thus our simulations predict an optimal rotation frequency, if preparations aim at smooth surfaces. This frequency also marks the frequency scale beyond which ripples do no longer appear. The very rapid crossover to non-ripple structures is in accordance with findings of Cui et al. (2005).

To compare our results with the prediction of Eq. 7.3 about the scaling of height with $\omega$, we studied $S=|h(\vec{k}, t)|^{2}$ for a fixed value of wavevector 
(a)

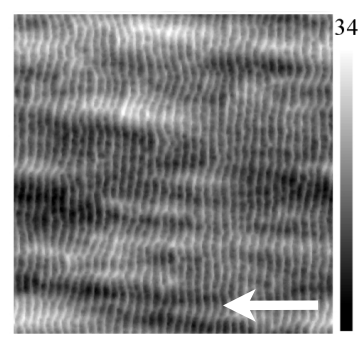

(b)
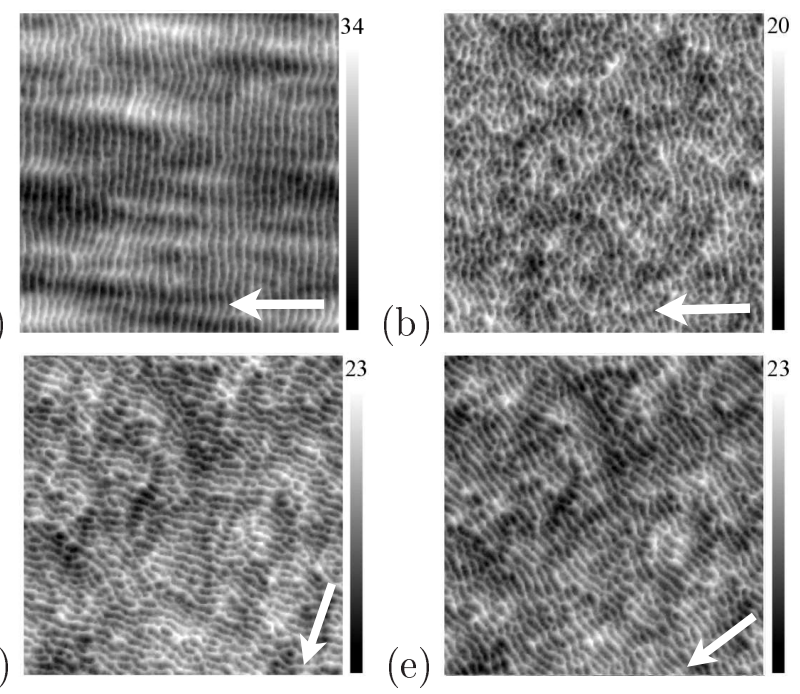

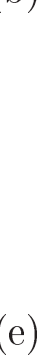

(c)
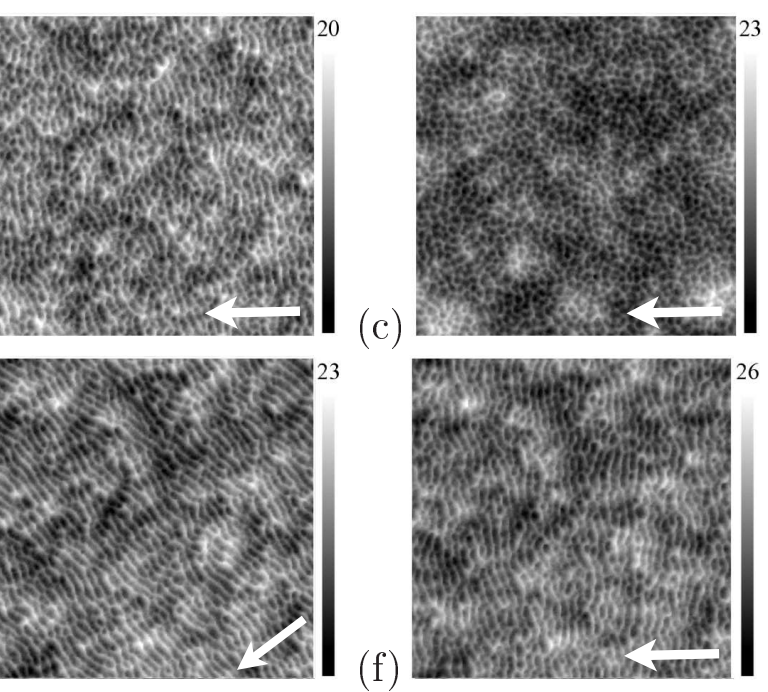

Figure 7.8: Snapshots of surface profile during RIBS (rotating IBS) at $t=40$ ions/atom for (a) $\omega=0$ (b) $\omega=\omega_{0}$ and (c) $\omega=7.5 \omega_{0}$. By increasing the rotation frequency, a transition of topographies from long parallel ripples to non-oriented cellular structures is observed. Panels (d-f) shots at $t=36,38$ and 40 ions/atom for rotating sample with frequency $\omega=0.5 \omega_{0}$, where ripples form and follow the orientation of the beam relative to the surface. Arrows indicate the instant direction of ion-beam.

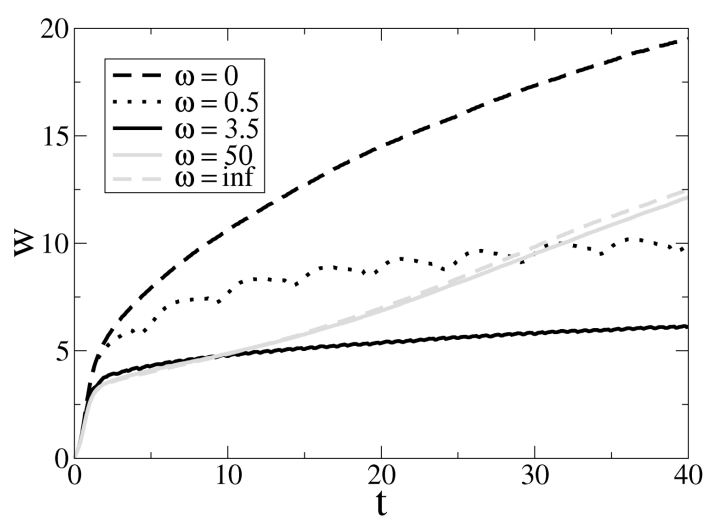

Figure 7.9: Time evolution of surface roughness during RIBS for different rotation frequencies. 


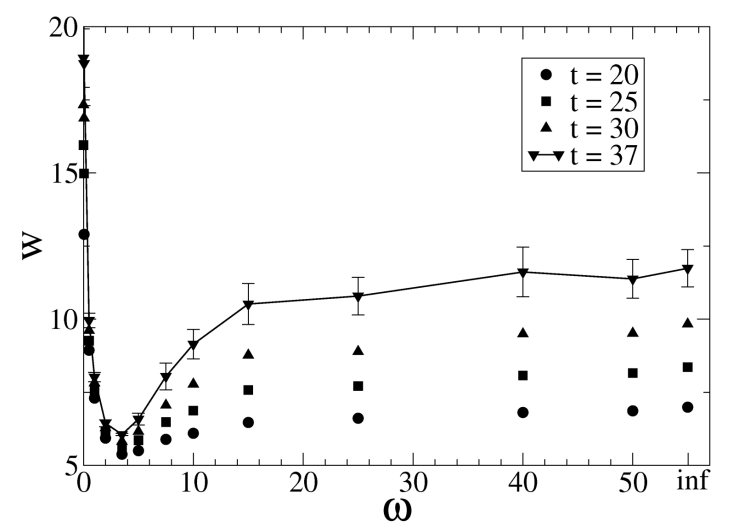

Figure 7.10: Roughness against angular frequency $\omega$ at different times.

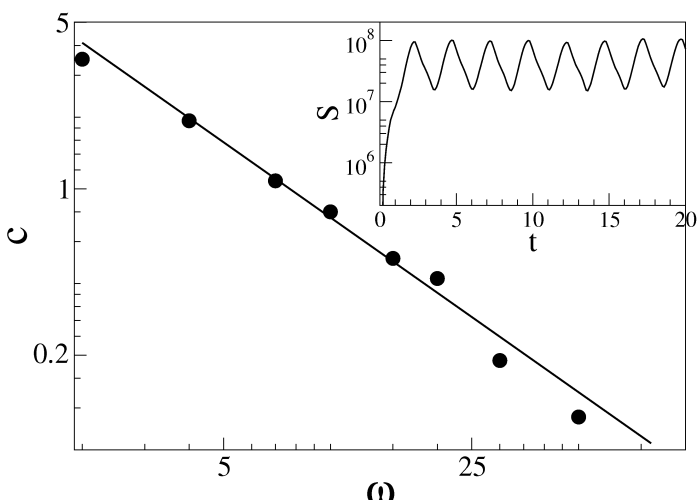

Figure 7.11: The logarithm of the oscillation amplitude of structure factor for a given $k$, vs. $\log \omega$. Inset: Time evolution of structure factor for a given $k$, for $\omega=2 \omega_{0}$ displays the oscillations. The amplitude of these oscillations is shown in the main figure.

$\vec{k}$. It grows rapidly and then oscillates with frequency $2 \omega$ around a saturation value with an $\omega$-dependent amplitude $c$, as shown in the inset of Fig. 7.11. The oscillatory behavior with frequency $2 \omega$ is also present in Eq. 7.3, The main part of the figure depicts the decrease of the oscillation amplitude with increasing $\omega$ in a double logarithmic plot. The fitted line has a slope of $-1.05 \pm 0.05$, which is very close to the $1 / \omega$ behavior suggested by Eq. 7.3. Note, however, that this equation was derived from linear theory and has to be multiplied by an exponential growth factor, whereas our result applies to the non-linear saturation regime. Due to the rapid initial growth, the regime of validity of Eq. 7.3 remained unobservable. 


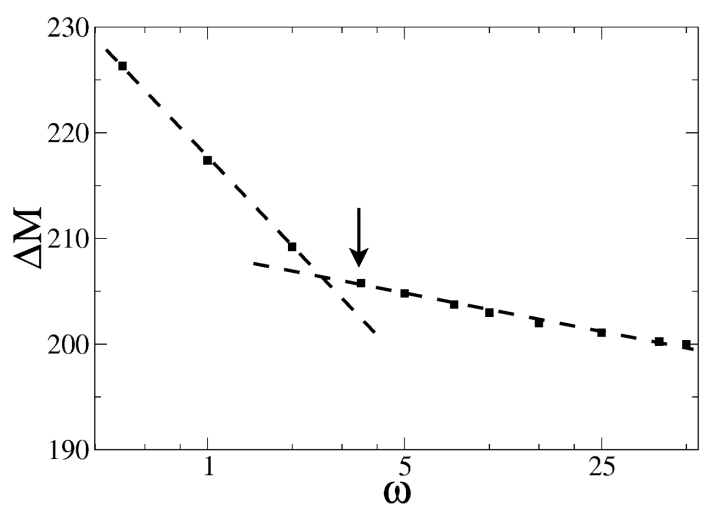

Figure 7.12: Total amount of eroded material (integrated yield) after 40 ions/atom of sputtering of rotating samples vs. rotation frequency. The arrow is at $\simeq 3.5 \omega_{0}$, the frequency which minimizes the total roughness.

We also measured the total amount of eroded material, $\Delta M$ up to $t=40$ ions/atom for different rotation frequencies. This integrated yield decreases with increasing $\omega$ and displays a clear distinction between a low- and a highfrequency regime in a semi-log plot, shown in Fig. 7.12, The interpolated crossover frequency between these regimes is very close to $\simeq 3.5 \omega_{0}$, the frequency, which minimizes the total roughness (see Fig. 7.10). This is in accordance with our findings (see Fig. (7.7) that more pronounced rippled prestructures (developing at low rotation frequencies at an angle $\omega t$ ) decay more rapidly (at $t+d t$ ) than the smaller and less regular height fluctuations, which appear at higher rotation frequencies. 


\section{Chapter 8}

\section{Conclusion}

In this work we have studied IBS-induced pattern formation on solid surfaces by kinetic MC simulations and continuum theories. We have addressed four questions, which concern basic physical mechanisms of the pattern formation seen in experiments, and have studied various proposals of techniques to improve, simplify or modify the pattern formation by special experimental setups. The basic questions we studied are

1. Is the interplay of erosion and surface diffusion comprehensively described by an isotropic Kuramoto-Sivashinsky equation or do different mechanisms of diffusion (and/or erosion) lead to different pattern scenarios, even if they would give rise to the same continuum description?

We have found evidence for the latter statement. Changing Sigmund's Gaussian energy deposition function to something, which is closer to BCA results, produces different (ripple) patterns for $\theta$ larger than $\sim 70^{\circ}$. Changing between different thermal diffusion mechanisms like Hamiltonian, bond-breaking or netbond-breaking, which all give rise to $\nabla^{4}$ term in continuum theory, changes the long-time behavior of ripple patterns. We have also systematically investigated the crossover from erosive to diffusive regime of pattern formation. In the diffusive regime, Ehrlich-Schwoebel barriers lead to new type of diffusion-driven patterns, but even there, the IBS pattern formation can not be completely understood as a "deposition of vacancies".

Related publication: 
- Hartmann, Kree, and Yasseri 2009, Simulating discrete models of pattern formation by ion-beam sputtering, J. Phys., 21224015.

2. Is it possible that a few, sub-mono-layer coverage of the surface with a second ("surfactant") atomic species has a significant influence on pattern formation?

We both extended our simulation model and presented a continuum model to include the effects of co-deposited surfactant atoms on the process of pattern formation. We demonstrated the possibility of inducing a large scale (micrometer) height gradient along the sputtered sample by redeposition of erosion-suppressive metallic atoms. The standard ripples form and evolve on top of this background large-scale structure. In KMC simulations as well as continuum theory we observed formation of ordered arrays of surfactants following the morphology of the rippled substrate. We show that a combination of clustering tendency and yield suppression, leads to the accumulation of surfactant clusters in the valleys of the ripples. A stronger yield suppression leads to stability of smooth surfaces and prohibits surface roughening, whereas a medium-sized sputtering suppression of yield leads to emergence of EhrlichSchwoebel induced patterns.

Related publication:

- Kree, Yasseri, and Hartmann 2009a, Surfactant Sputtering: Theory of a new method of surface nano-structuring by ion beams, Nucl. Ins. Meth. Phys. B, 2671403.

- Hartmann, Kree, and Yasseri 2009, Simulating discrete models of pattern formation by ion-beam sputtering, J. Phys., 21224015.

\section{Do orientational fluctuations of ion-beam trajectories (beam di- vergence) have effects on the pattern formation?}

We have set up and studied a continuum theory and a Monte Carlo model of IBS including ion-beam profiles. In both approaches we have found clear indications of a rather strong dependence of surface patterns upon the special type of noise, which is produced by non-trivial ion-beam profiles, as has been observed in experiments (Ziberi et al. 2004; Ziberi et al. 2008). Whereas the 
continuum approach is most effective for small, Gaussian fluctuations, where it leads to a renormalization of coefficients of the local evolution equation of the average height profile, the Monte Carlo model is able to treat generic as well as more realistic and material-specific situations. As the pattern forming scenarios depend sensitively on beam parameters and diffusion, such a modeling is necessary, if one wishes to compare theoretical and experimental results in more detail. Our KMC simulations show that in normal incidence angle sputtering, different length scales can be selected and grow by including or excluding the beam-noise. In grazing incidence angle, more regular ripples emerge when the beam has a wide profile. In high temperature regimes, presence of the beam-noise favors the Ehrlich-Schwoebel effects and changes the morphology of patterns towards diffusion induced patterns.

Related publication:

- Kree, Yasseri, and Hartmann 2009b, The influence of beam divergence on ion-beam induced surface patterns ,Nucl. Ins. Meth. Phys. B, 267 1407.

\section{Is it possible to improve, simplify or modify IBS-induced pattern formation by multiple-beams or rotated-beam setups?}

We studied dual ion beam sputtering (DIBS), sequential ion beam sputtering (SIBS) and rotating ion beam sputtering (RIBS) by a kinetic MC simulation technique, which combines erosion events due to single ions and surface diffusion. For a DIBS setup with two diametrically opposed beams, we did not confirm predictions by Carter (2005), but rather found non-moving ripples with orientations as in a single ion beam setup. The ripples have a higher degree of order and more symmetrical slopes as compared to those created by single beam sputtering. For DIBS setups with crossed ion-beams, we found ripple patterns for crossing angles $\Delta \phi \neq 90^{\circ}$ and square patterns for crossing of balanced beams at exactly right angle. The ripple orientations follow the predictions from linear Bradley Harper theory. Any kind of beam-imbalance leads to ripple patterns oriented according to the dominant beam. This is in accordance with the experimental observation by Joe et al. (2009). For SIBS setups, we found a very rapid destruction of the ripple pre-structure of the previous rotation step, which cannot be explained by linear Bradley-Harper 
theory. The flattening of the pre-structure leads to a transient decrease in total roughness. Only within a very short time window, the growing new generation of ripples and the shrinking old ones lead to a superposed square pattern. Thus we could not confirm propositions to use SIBS as a universal substitute for complicated multi-beam setups. The rapid destruction of the pre-structure is in accordance with findings of Joe et al. (2009). For RIBS setups we observed a non-monotonic dependence of roughness upon rotation frequency. At a frequency scale set by the structural relaxation time of prestructures, which can be observed in SIBS simulations, an increasingly pronounced minimum of roughness occurs with increasing time. We found that the structure factor at fixed wavevector rapidly approaches stationary oscillations around a saturation value with oscillation amplitudes inversely proportional to frequency. This behavior was also predicted from linear theory, but seems to have a much broader range of validity.

Related publication:

- Yasseri and Kree, A Monte Carlo study of surface sputtering by dual and rotated ion beams, submitted to Nucl. Ins. Meth. Phys. B.

\section{Outlook}

Our results show that the considered mechanisms have significant influence on the IBS-induced pattern formation, which can be used to improve surface engineering. On the other hand, none of the mechanisms lead to patterns of a quality, uniformity and regularity as has been observed in some recent experiments.

In fact, two potentially important physical mechanisms have not been considered in this work: elastic interactions between surface and substrate and redeposition of eroded atoms. The inclusion of these mechanisms requires substantial extensions both of the KMC model and of the continuum theory, but seems necessary to make further progress in the case of IBS-induced pattern formation. 


\section{Appendix A}

\section{Implementation of directional noise in growth equation}

We start from Eq. 6.3 with $\hat{L}_{0}$ and $\hat{L}_{1}$ given by

$$
J_{0}=\hat{L}_{0} \tilde{h}=v_{0}^{\prime}\left(\theta_{0}\right) \frac{\partial \tilde{h}(x, y, t)}{\partial x}+\nu_{x} \frac{\partial^{2} \tilde{h}(x, y, t)}{\partial x^{2}}+\nu_{y} \frac{\partial^{2} \tilde{h}(x, y, t)}{\partial y^{2}}-B \nabla^{2} \nabla^{2} \tilde{h}
$$

therefore,

$$
J_{1}=\hat{L}_{1} \tilde{h}=v_{0}^{\prime \prime}\left(\theta_{0}\right) \frac{\partial \tilde{h}(x, y, t)}{\partial x}+\nu_{x}^{\prime} \frac{\partial^{2} \tilde{h}(x, y, t)}{\partial x^{2}}+\nu_{y}^{\prime} \frac{\partial^{2} \tilde{h}(x, y, t)}{\partial y^{2}}
$$

Note that, for simplicity, we put $\tilde{h} \rightarrow h$ in this appendix and prime indicate $\partial /\left.\partial \theta\right|_{\theta=\theta_{0}}$. The term $\delta \theta v_{0}^{\prime}$, which appears in $\hat{L}_{1}$ is an additive noise and its average is zero, therefore it does not contribute to $\partial_{t}\langle h\rangle$ so we neglect it.

We assume a Gaussian noise which is white in time

$$
\left\langle\delta \theta(\mathbf{r}, t) \delta \theta\left(\mathbf{r}^{\prime}, t^{\prime}\right)\right\rangle=C\left(\left|\mathbf{r}-\mathbf{r}^{\prime}\right|\right) \delta\left(t-t^{\prime}\right)
$$

We integrate over small time step $\Delta t$

$$
h(\mathbf{r}, t+\Delta t)-h(\mathbf{r}, t)=J_{0}(t) \Delta t+\int_{t}^{t+\Delta t} \delta \theta\left(\mathbf{r}, t^{\prime}\right) J_{1}\left(t^{\prime}\right) d t^{\prime}
$$

and look for contributions to $\langle\Delta h\rangle=\langle h(\mathbf{r}, t+\Delta t)-h(\mathbf{r}, t)\rangle$ of $\mathcal{O}(\Delta t)$. Then we divide by $\Delta t$ and take the limit $\Delta t \rightarrow 0$. Therefore we approximate the first term on the right hand side to $\mathcal{O}(\Delta t)$. It needs no further treatment. Consider the second term in the right hand side $A$ :

$$
A=\int_{t}^{t+\Delta t} \delta \theta\left(\mathbf{r}, t^{\prime}\right) J_{1}\left(\nabla h\left(\mathbf{r}, t^{\prime}\right), \nabla^{2} h\left(\mathbf{r}, t^{\prime}\right), \ldots\right) d t^{\prime} .
$$


To extract $\mathcal{O}(\Delta t)$-term in the average we have to insert

$$
h\left(\mathbf{r}, t^{\prime}\right)=h(\mathbf{r}, t)+J_{0}(t)\left(t-t^{\prime}\right)+\int_{t}^{t^{\prime}} \delta \theta\left(\mathbf{r}, t^{\prime \prime}\right) J_{1}\left(t^{\prime \prime}\right) d t^{\prime \prime} .
$$

The neglected terms will be of higher order in $\Delta t$ after averaging, so they need not be taken into account. The term $\propto J_{0}(t)$ will also not contribute to $\mathcal{O}(\Delta t)$ after averaging and is dropped from here on. So we have to use $h\left(\mathbf{r}, t^{\prime}\right)=h(\mathbf{r}, t)+\delta h\left(t^{\prime}\right)$ where $\delta h\left(t^{\prime}\right)=\int_{t}^{t^{\prime}} \delta \theta\left(\mathbf{r}, t^{\prime \prime}\right) J_{1}\left(t^{\prime \prime}\right) d t^{\prime \prime}$. We expand $J_{1}\left(t^{\prime}\right)$ in $\delta h$ (only the linear term contributes).

$$
\begin{array}{r}
J_{1}\left(\nabla h(\mathbf{r}, t)+\nabla \delta h(\mathbf{r}, t), \nabla^{2} h(\mathbf{r}, t)+\nabla^{2} \delta h(\mathbf{r}, t)\right)=J_{1}(t) \\
+\frac{\partial J_{1}(t)}{\partial \vec{\nabla} h(\mathbf{r}, t)} \vec{\nabla} \delta h(\mathbf{r}, t)+\frac{\partial J_{1}(t)}{\partial\left(\nabla^{2} h(\mathbf{r}, t)\right)} \nabla^{2} \delta h(\mathbf{r}, t)
\end{array}
$$

and insert in the $A$ above:

$$
A=\int_{t}^{t+\Delta t} \delta \theta\left(\mathbf{r}, t^{\prime}\right)\left[J_{1}\left(t^{\prime}\right)+\frac{\partial J_{1}\left(t^{\prime}\right)}{\partial \vec{\nabla} h\left(\mathbf{r}, t^{\prime}\right)} \vec{\nabla} \delta h(\mathbf{r}, t)+\frac{\partial J_{1}\left(t^{\prime}\right)}{\partial\left(\nabla^{2} h\left(\mathbf{r}, t^{\prime}\right)\right)} \nabla^{2} \delta h\left(\mathbf{r}, t^{\prime}\right)\right] d t^{\prime}
$$

$J_{1}\left(t^{\prime}\right)$ vanishes after averaging. So

$$
\begin{aligned}
A=\int_{t}^{t+\Delta t} & \delta \theta\left(\mathbf{r}, t^{\prime}\right)\left\{\frac{\partial J_{1}\left(t^{\prime}\right)}{\partial \vec{\nabla} h\left(\mathbf{r}, t^{\prime}\right)} \cdot \vec{\nabla} \int_{t}^{t^{\prime}} \delta \theta\left(\mathbf{r}, t^{\prime \prime}\right) J_{1}\left(t^{\prime \prime}\right) d t^{\prime \prime}\right. \\
& \left.+\frac{\partial J_{1}\left(t^{\prime}\right)}{\partial\left(\nabla^{2} h\left(\mathbf{r}, t^{\prime}\right)\right)} \nabla^{2} \int_{t}^{t^{\prime}} \delta \theta\left(\mathbf{r}, t^{\prime \prime}\right) J_{1}\left(t^{\prime \prime}\right) d t^{\prime \prime}\right\} d t^{\prime}
\end{aligned}
$$

Note that $J_{1}$ depends on $\mathbf{r}$ and therefore $\nabla$ and $\nabla^{2}$ act both on $\delta \theta$ and $J_{1}$.

Now we do the average. but before that note:

(i) $\left\langle\delta \theta\left(t^{\prime}\right) \delta \theta\left(t^{\prime \prime}\right)\right\rangle=\delta\left(t^{\prime}-t^{\prime \prime}\right)$ gives a $\delta$-function at the upper limit of the integrals over $t^{\prime \prime}$. From stochastic calculus (Wong and Zakai 1965) we know the correct interpretation for physical noise is

$$
\int_{t}^{t^{\prime}} d t^{\prime \prime}\left\langle\delta \theta\left(t^{\prime}\right) \delta \theta\left(t^{\prime \prime}\right)\right\rangle=\lim _{\epsilon \rightarrow 0} \frac{1}{2} \int_{t}^{t^{\prime}+\epsilon} d t^{\prime \prime} \delta\left(t^{\prime}-t^{\prime \prime}\right) .
$$

The factor of $1 / 2$ comes from the contributions of the "left half" of the regularized $\delta$-function.

(ii) $\vec{\nabla}$ and $\nabla^{2}$ give several terms. Consider

$$
\int_{t}^{t+\Delta t} \int_{t}^{t^{\prime}} \frac{\partial J_{1}\left(t^{\prime}\right)}{\partial \vec{\nabla} h\left(\mathbf{r}, t^{\prime}\right)}\left\langle\delta \theta\left(\mathbf{r}, t^{\prime}\right) \vec{\nabla}\left\{\delta \theta\left(\mathbf{r}, t^{\prime \prime}\right)\right\rangle J_{1}\left(t^{\prime \prime}\right) d t^{\prime \prime}\right.
$$


which equals to

$$
\int_{t}^{t+\Delta t} \int_{t}^{t^{\prime}+\epsilon} \frac{\partial J_{1}\left(t^{\prime}\right)}{\partial \vec{\nabla} h\left(\mathbf{r}, t^{\prime}\right)}\left\{\left\langle\delta \theta\left(\mathbf{r}, t^{\prime}\right) \vec{\nabla}_{\mathbf{r}} \delta \theta\left(\mathbf{r}, t^{\prime \prime}\right)\right\rangle J_{1}\left(t^{\prime \prime}\right)+\left\langle\delta \theta\left(t^{\prime}\right) \delta \theta\left(t^{\prime \prime}\right)\right\rangle \vec{\nabla} J_{1}\left(t^{\prime \prime}\right)\right\} .
$$

The first term:

$$
\lim _{\mathbf{r}^{\prime} \rightarrow \mathbf{r}} \vec{\nabla}_{\mathbf{r}}\left\langle\delta \theta\left(\mathbf{r}^{\prime}\right) \delta \theta(\mathbf{r})=\left.\lim _{\mathbf{r}^{\prime} \rightarrow \mathbf{r}} \vec{\nabla}_{\mathbf{r}} C\left(\left|\mathbf{r}-\mathbf{r}^{\prime}\right|\right)\right|_{\mathbf{r}=0} \frac{1}{2} \delta\left(t-t^{\prime}\right)\right.
$$

which is

$$
\vec{\nabla}_{\mathbf{r}} C(r)_{r=0} \frac{1}{2} \delta\left(t-t^{\prime}\right)
$$

and the second term: $\left\langle\delta \theta\left(t^{\prime}\right) \delta \theta\left(t^{\prime \prime}\right)\right\rangle=(1 / 2) C(0) \delta\left(t^{\prime}-t^{\prime \prime}\right)$, Note that usually $C(0)$ is maximum of smooth $C(r)$ so we neglect $(\nabla C)(0)$. Thus we get

$$
A=\frac{\Delta t}{2} C(0) \frac{\partial J_{1}}{\partial \vec{\nabla} h} \cdot \vec{\nabla} J_{1}+\frac{\partial J_{1}}{\partial\left(\nabla^{2} h\right)}\left(\frac{\Delta t}{2} C(0) \nabla^{2} J_{1}+\frac{\Delta t}{2}\left(\nabla^{2} C\right)(0) J_{1}\right) .
$$

This gives the averaged evolution equation as

$$
\frac{\partial\langle h\rangle}{\partial t}=J_{0}+\frac{C(0)}{2} \frac{\partial J_{1}}{\partial \vec{\nabla} h} \cdot \vec{\nabla} J_{1}+\sum_{i=1}^{2} \frac{\partial J_{1}}{\partial\left(\partial_{i}^{2} h\right)}\left(\frac{C(0)}{2} \partial_{i}^{2} J_{1}+\frac{1}{2}\left(\partial_{i}^{2} C\right)(0) J_{1}\right)
$$

Here we have generalized to $J\left(\nabla h, \partial_{x}^{2}, \partial_{y}^{2}\right)$ because we want to plug in the Bradley-Harper model.

\section{Plugging of Bradley Harper theory}

$$
\frac{\partial J_{1}}{\partial\left(\partial_{x}\right) h}=v^{\prime \prime}, \frac{\partial J_{1}}{\partial\left(\partial_{y}\right) h}=0
$$

and

$$
\frac{\partial J_{1}}{\partial\left(\partial_{x}^{2}\right) h}=\nu_{x}^{\prime}, \frac{\partial J_{1}}{\partial\left(\partial_{y}^{2} h\right)}=\nu_{y}^{\prime}
$$

therefore

$\frac{\partial\langle h\rangle}{\partial}=J_{0}+\frac{C(0)}{2} v_{0}^{\prime \prime} \partial_{x} J_{1}+\nu_{x}^{\prime}\left(\frac{C(0)}{2} \partial_{x}^{2} J_{1}+\frac{\left(\partial_{x}^{2} C\right)(0) J_{1}}{2}\right)+\nu_{y}^{\prime}\left(\frac{C(0)}{2} \partial_{y}^{2} J_{1}+\frac{\left(\partial_{y}^{2} C\right)(0) J_{1}}{2}\right)$

Now we look for the terms $\propto \partial_{x} h, \partial_{y} h, \partial_{x}^{2} h$ and $\partial_{y}^{2} h$.

$$
\begin{gathered}
\frac{C(0)}{2} \nu_{0}^{\prime \prime} \partial_{x} J_{1}=\frac{C(0)}{2} v_{0}^{\prime \prime} \partial_{x} h+\mathcal{O}\left(\partial^{3}\right) \\
\partial_{x}^{2} \frac{C(0)}{2} \nu_{x}^{\prime} J_{1}=\frac{\partial_{x}^{2} C(0)}{2} \nu_{x}^{\prime}\left[\nu_{x}^{\prime} \partial_{x}^{2}+\nu_{y}^{\prime} \partial_{y}^{2}\right] h+\frac{\partial_{x}^{2} C(0)}{2} \nu_{x}^{\prime} v_{0}^{\prime \prime} \partial_{x} h .
\end{gathered}
$$




$$
\partial_{y}^{2} \frac{C(0)}{2} \nu_{y}^{\prime} J_{1}=\frac{\partial_{y}^{2} C(0)}{2} \nu_{x}^{\prime}\left[\nu_{y}^{\prime} \partial_{x}^{2}+\nu_{y}^{\prime} \partial_{y}^{2}\right] h+\frac{\partial_{y}^{2} C(0)}{2} \nu_{y}^{\prime} v_{0}^{\prime \prime} \partial_{y} h
$$

The terms $\frac{C(0)}{2} \partial_{x}^{2} J_{1}$ and $\frac{C(0)}{2} \partial_{y}^{2} J_{1}$ are of $\mathcal{O}\left(\partial^{3}\right)$. Thus:

$$
\begin{aligned}
\frac{\langle h\rangle}{\partial t}=-v\left(\theta_{0}\right)+ & {\left[v_{0}^{\prime}+\frac{\partial_{x}^{2} C(0)}{2} \nu_{x}^{\prime} v_{0}^{\prime \prime}+\frac{\partial_{y}^{2} C(0)}{2} \nu_{y}^{\prime} v_{0}^{\prime \prime}\right] \partial_{x} h } \\
& +\left[\nu_{x}+\frac{\partial_{x}^{2} C(0)}{2} \nu_{x}^{\prime} \nu_{x}^{\prime}+\frac{\partial_{y}^{2} C(0)}{2} \nu_{y}^{\prime} \nu_{x}^{\prime}\right] \partial_{x}^{2} h \\
& +\left[\nu_{y}+\frac{\partial_{x}^{2} C(0)}{2} \nu_{x}^{\prime} \nu_{y}^{\prime}+\frac{\partial_{y}^{2} C(0)}{2} \nu_{y}^{\prime} \nu_{y}^{\prime}\right] \partial_{y}^{2} h
\end{aligned}
$$

Since we take an isotropic correlation function: $\partial_{x}^{2} C=\partial_{y}^{2} C=C^{\prime \prime}$, so

$$
\begin{gathered}
v_{0}^{\prime R}=v_{0}^{\prime}+\frac{C^{\prime \prime}}{2}\left(\nu_{x}^{\prime}+\nu_{y}^{\prime}\right) v_{0}^{\prime \prime} \\
\nu_{x}^{R}=\nu_{x}+\frac{C^{\prime \prime}}{2}\left(\nu_{x}^{\prime}+\nu_{y}^{\prime}\right) \nu_{x}^{\prime}+\frac{C}{2} v_{0}^{\prime \prime 2} \\
\nu_{y}^{R}=\nu_{y}+\frac{C^{\prime \prime}}{2}\left(\nu_{x}^{\prime}+\nu_{y}^{\prime}\right) \nu_{y}^{\prime}
\end{gathered}
$$




\section{References}

Alkemade, P. F. A. (2006). Physical Review Letters 96 (10), 107602.

Aste, T. and U. Valbusa (2005). New Journal of Physics 7, 122.

Barabási, A.-L. and H. Stanley (1995). Fractal Concepts in Surface Growth. Cambridge: Cambridge University Press.

Bartosz Liedke, Karl-Heinz Heinig, S. F. W. M. (2009). Abstract book of Nanoscale Modification of Surfaces and Thin Films.

Berg, S., A. M. Barklund, B. Gelin, C. Nender, and I. Katardjiev (1992). Volume 10, pp. 1592. AVS.

Bradley, R. M. (1996). Phys. Rev. E 54, 6149.

Bradley, R. M. and E.-H. Cirlin (1996). Appl. Phys. Lett. 68, 3722.

Bradley, R. M. and J. M. E. Harper (1988). J. Vac. Sci. Technol. A 6(4), 2390.

Brown, A.-D. and J. Erlebacher (2005). Phys. Rev. B 72(7), 075350.

Burger, M. (2006). Commun. Math. Sci 4 (1), 1.

Carter, G. (1998). Vacuum 49, 285.

Carter, G. (2004). Vacuum 7\%, 97.

Carter, G. (2005). Vacuum 79, 106.

Carter, G. (2006). Vacuum 81, 138.

Carter, G. and V. Vishnyakov (1996). Phys. Rev. B 54(24), 17647-17653.

Castro, M., R. Cuerno, L. Vázquez, and R. Gago (2005). Phys. Rev. Lett. $94(1), 016102$.

Cermelli, P., E. Fried, and M. Gurtin (2005). J. Fluid. Mech 544, 339. 
Chan, W.-L. and E. Chason (2007). Journal of Applied Physics 101(12), 121301.

Chason, E., W. L. Chan, and M. S. Bharathi (2006). Physical Review $B 74(22), 224103$.

Cuerno, R. and A.-L. Barabási (1995). Phys. Rev. Lett. 74(23), 4746.

Cui, B., P. I. Cohen, A. M. Dabiran, and R. Jorgenson (2005). J. Appl. Phys. 98, 083504.

Das Sarma, S. and P. Tamborenea (1991). Phys. Rev. Lett. 66 (3), 325.

Edwards, S. F. and D. R. Wilkinson (1982). Proc. R. Soc. A 381, 17.

Facsko, S., T. Dekorsy, C. Koerdt, C. Trappe, H. Kurz, A. Vogt, and H. L. Hartnagel (1999). Science 285 (5433), 1551-1553.

Family, F. (1986). Journal of Physics A 19(8), L441.

Farmery, B. W. and M. W. Thompson (1968). Phil. Mag. 18(152), 415.

Feix, M. (2002). Simulation von Einzelioneneinschlägen auf Kupfer. Master's thesis, University of Göttingen, Germany.

Feix, M., A. K. Hartmann, R. Kree, J. Muñoz-García, and R. Cuerno (2005). Physical Review B 71 (12), 125407.

Feynman, R. (1960). Caltech's Engineering and Science, 22.

Flamm, D., F. Frost, and D. Hirsch (2001). Applied Surface Science 179(14), $95-101$.

Floro, J. A., B. K. Kellerman, E. Chason, S. T. Picraux, D. K. Brice, and K. M. Horn (1995). Journal of Applied Physics 77(6), 2351-2357.

Frost, F. (2002). Appl. Phys. A $74(1), 131$.

Frost, F., R. Fechner, D. Flamm, B. Ziberi1, W. Frank, and A. Schindler (2004). Applied Physics A 78(5), 651.

Frost, F., R. Fechner, B. Ziberi, J. Vollner, D. Flamm, and A. Schindler (2009). Journal of Physics 21(22), 224026 (20pp).

Frost, F. and B. Rauschenbach (2003). Appl. Phys. A r7, 1.

Frost, F., A. Schindler, and F. Bigl (2000). Phys. Rev. Lett. 85 (19), 41164119. 
Frost, F., B. Ziberi, T. Höche, and B. Rauschenbach (2004). Nucl. Instrum. Methods Phys. Res. B 216, 9-19.

Frost, F., B. Ziberi, A. Schindler, and B. Rauschenbach (2008). Appl. Phys. A 91,551 .

Gago, R., L. Vázquez, R. Cuerno, M. Varela, C. Ballesteros, and J. M. Albella (2001). Applied Physics Letters 78(21), 3316-3318.

Garcia-Ojalvo, J. and J. Sancho (1985). Noise in spatially extended systems. New York: Springer.

Habenicht, S., W. Bolse, K. P. Lieb, K. Reimann, and U. Geyer (1999). Phys. Rev. B 60 (4), R2200-R2203.

Habenicht, S., K. P. Lieb, J. Koch, and A. D. Wieck (2002). Phys. Rev. B $65(11), 115327$.

Hartmann, A. K., R. Kree, U. Geyer, and M. Koelbel (2002). Phys. Rev. B 65, 193403.

Hartmann, A. K., R. Kree, and T. Yasseri (2009). J. Phys.: Condens. Matter 21, 224015.

Headrick, R. L. and H. Zhou (2009). Journal of Physics 21(22), 224005 (12pp).

Hofsäss, H. and K. Zhang (2008). Appl. Phys. A 92(3), 517.

Hofsäss, H. and K. Zhang (2009). Nucl. Ins. Meth. Phys. B 267(16), 2731.

Hofsäss, H., K. Zhang, and H. Zutz (2009). Nucl. Ins. Meth. Phys. B 267(89), 1398.

Šmilauer, P., M. R. Wilby, and D. D. Vvedensky (1993). Phys. Rev. B $47(7)$, 4119.

Joe, M., C. Choi, B. Kahng, J.-H. Kim, and J.-S. Kim (2009). J. Phys.: Condens. Matter 21, 224011.

Joe, M., C. Choi, B. Kahng, C. Y. Kwak, and J.-S. Kim (2008). J. Korean Phys. Soc. 52, S181.

Johnson, M. D., C. Orme, A. W. Hunt, D. Graff, J. Sudijono, L. M. Sander, and B. G. Orr (1994). Phys. Rev. Lett. 72(1), 116. 
Kim, J. M. and S. Das Sarma (1994). Phys. Rev. Lett. 72(18), 2903.

Konarski, P. and M. Hautala (1995). Vacuum 47, 1111.

Kong, K.-j., H. W. Yeom, D. Ahn, H. Yi, and B. D. Yu (2003). Phys. Rev. B $67(23), 235328$.

Koponen, I., M. Hautala, and O.-P. Sievänen (1997). Phys. Rev. Lett. 78(13), 2612-2615.

Koponen, I., O.-P. Sievanen, M. Hautala, and M. Hakovirta (1997). J. Appl. Phys. 94, 262.

Kree, R., T. Yasseri, and A. Hartmann (2009a). Nuclear Ins. Meth. Phys. B 267(8-9), $1403-1406$.

Kree, R., T. Yasseri, and A. Hartmann (2009b). Nuclear Ins. Meth. Phys. B 267(8-9), 1407 - 1411.

Krug, J. (1995). Habilitation thesis, Juelich, Duesseldorf.

Krug, J., M. Plischke, and M. Siegert (1993). Phys. Rev. Lett. 70 (21), 3271.

Kuramoto, Y. and T. Tsuzuki (1976). Prog. Theor. Phys. $55(2), 356$.

Lai, Z.-W. and S. Das Sarma (1991). Phys. Rev. Lett. 66(18), 2348.

Makeev, M. A., R. Cuerno, and A. L. Barabási (2002). Nucl. Instr. and Meth. in Phys. Res. B 197(3-4), 185.

Maynard, A. D., R. J. Aitken, T. Butz, V. Colvin, K. Donaldson, G. Oberdorster, M. A. Philbert, J. Ryan, A. Seaton, V. Stone, S. S. Tinkle, L. Tran, N. J. Walker, and D. Warheit (2006). Nature 444, 267.

Moseler, M., P. Gumbsch, C. Casiraghi, A. C. Ferrari, and J. Robertson (2005). Science 309 (5740), 1545-1548.

Muñoz-García, J., R. Cuerno, and M. Castro (2009). J. Phys.: Condens. Matter 21(22), 224020.

Muñoz-García, J., R. Cuerno, J. A. Sánchez-García, M. Castro, and R. Gago (2009). Self-organized surface nanopatterning by ion beam sputtering. New York: Springer.

Navez, M., C. Sella, and C. Chaperot (1962). Compte. Rend. 254, 240. 
Nordlund, K., M. Ghaly, R. S. Averback, M. Caturla, T. Diaz de la Rubia, and J. Tarus (1998). Phys. Rev. B 57(13), 7556-7570.

Novikov, E. A. (1965). Sov. Phys. JETP 20, 1290.

Oates, T. W. H., A. Keller, S. Facsko, and A. Mücklich (2007). Plasmonics $2(2), 47$.

Park, S., B. Kahng, H. Jeong, and A.-L. Barabási (1999). Phys. Rev. Lett. $83(17), 3486$.

Reichel, F., L. P. H. Jeurgens, G. Richter, P. van Aken, and E. J. Mittemeijer (2007). Acta Materialia 55, 6027.

Robinson, M. T. (1994). Rad. Effects and Defects in Solids $130(3)$.

Robinson, M. T. and I. M. Torrens (1974). Phys. Rev. B 9(12), 5008.

Rost, M. and J. Krug (1995). Phys. Rev. Lett. 75 (21), 3894.

Shenoy, V. B., W. L. Chan, and E. Chason (2007). Physical Review Letters $98(25), 256101$.

Siegert, M. and M. Plischke (1992). Phys. Rev. Lett. 68(13), 2035.

Siegert, M. and M. Plischke (1994). Phys. Rev. E 50(2), 917-931.

Sigmund, P. (1969). Phys. Rev. 184 (2), 383-416.

Sigmund, P. (1973). J. Mat. Sci. 8, 1545.

Sivashinsky, G. L. (1977). Acta Astronautica 4, 1177.

Stepanova, M. and S. K. Dew (2006). J. Vac. Sci. Tech. B $24(2), 592$.

Stepanova, M., S. K. Dew, and I. P. Soshnikov (2005). Applied Physics Letters $86(7), 073112$.

Tamborenea, P. I. and S. Das Sarma (1993). Phys. Rev. E 48(4), 2575.

Tanemura, M., S. Aoyama, and F. Okuyama (1992). Surf. Interface. Anal. 18, 475.

Thompson, M. W. (1968). Phil. Mag. 18, 152.

Thürmer, K., R. Koch, M. Weber, and K. H. Rieder (1995). Phys. Rev. Lett. $75(9), 1767-1770$.

Tok, E. S., S. W. Ong, and H. C. Kang (2004). Phys. Rev. E $70(1), 011604$. 
Valbusa, U., C. Boragno, and F. B. de Mongeot (2002). J. Phys.: Condens. Matter 14, 8153-8175.

Villain, J. and A. Pimpinelli (1994). Physique de la croissance cristalline. Paris: Aléa-Saclay-Eyrolle.

Vogel, S. and S. J. Linz (2007). Phys. Rev. B 75, 085425.

Wolf, D. E. and J. Villain (1990). Europhys. Lett. 13, 389.

Wong, E. and M. Zakai (1965). Ann. Math. Statst. 36, 1560.

Yasseri, T. and R. Kree. Submitted to Nucl. Ins. Meth. Phys. B.

Yewande, E. O., R. Kree, and A. K. Hartmann (2005). Phys. Rev. B 71, 195405 .

Yewande, E. O., R. Kree, and A. K. Hartmann (2006). Phys. Rev. B 73, 115434.

Yewande, E. O., R. Kree, and A. K. Hartmann (2007). Phys. Rev. B 75, 155325 .

Zalar, A. (1985). Thin Solid Films 124, 223.

Zalar, A. (1986). Surf. Interface Anal. 9, 41.

Ziberi, B., M. Cornejo, F. Frost, and B. Rauschenbach (2009). Journal of Physics 21 (22), 224003 (17pp).

Ziberi, B., F. Frost, T. Höche, and B. Rauschenbach (2005). Physical Review B 72(23), 235310.

Ziberi, B., F. Frost, and B. Rauschenbach (2006). Applied Physics Letters $88(17), 173115$.

Ziberi, B., F. Frost, M. Tartz, H. Neumann, and B. Rauschenbach (2004). Thin Solid Films 459, 106.

Ziberi, B., F. Frost, M. Tartz, H. Neumann, and B. Rauschenbach (2008). Appl. Phys. Lett. 92, 063102.

Ziegeler, J. F., J. P. Biersack, and K. Littmark (1985). The Stopping and Range of Ions in Matter. New York: Pergamon. see also http: //www.srim.org/ 


\section{Curriculum vitae}

\section{TAHA YASSERI}

Birth: 09. Sep. 1984, Tehran

Institut für Theoretische Physik

Nationality: Iranian

Friedrich-Hund-Platz 1

Marital status: single

37077 Göttingen, Germany

\section{EDUCATION}

- PhD Candidate, Physics,

Faculty of Physics, Georg-August-Universität

Göttingen, Germany.

Dec. 2006 - Present

Adviser: Prof. Dr. R. Kree, Co-adviser: Prof. Dr. A.K. Hartmann

- Master of Science, Physics,

Department of Physics, Sharif University of Technology

Tehran, Iran.

Sep. 2005 - Sep. 2006

Topic: Localization in scale free networks; Marked 19.50/20

Adviser: Prof. Dr. M. R. R. Tabar

- Bachelor of Science, Physics,

Department of Physics, Sharif University of Technology

Tehran, Iran.

Sep. 2002 - Jul. 2005

\section{PUBLICATIONS}

1. "A Monte Carlo study of surface sputtering by dual and rotated ion beams", with R. Kree, Submitted to Nucl. Ins. Meth. B

2. "Simulating discrete models of pattern formation by ion-beam sputtering", with A.K. Hartmann and R. Kree, J. Phys. 21, 224015 (2009). 
3. "Surfactant Sputtering: Theory of a new method of surface nano-structuring by ion beams", with R. Kree and A.K. Hartmann, Nucl. Ins. Meth. B 267, 1403 (2009).

4. "The influence of beam divergence on ion-beam induced surface patterns", with R. Kree and A.K. Hartmann, Nucl. Ins. Meth. B 267, 1407 (2009).

\section{CONFERENCE PRESENTATIONS (11 contributions)}

- Nanoscale Modification of Surfaces and Thin Films, Rathen, Germany, Sep. '09. (POSTER)

- Spring meeting of the German Physics Society, Dresden, Germany, March '09. (TWO SHORT TALKS AND TWO POSTERS)

- 16th International Conference on Ion-Beam Modification of Materials, Dresden, Germany, Sep '08. (TWO POSTERS)

- NanoSteps, Selforganized nanostructures on crystal surfaces, Corsica, France, June '08. (SHORT TALK)

- Spring meeting of the German Physics Society, Berlin, Germany, February '08. (SHORT TALK)

- Conference on Computational Physics, Brussels, Belgium, September '07. (POSTER)

- The 23rd International Conference on Statistical Physics, Genova, Italy, July '07. (POSTER)

\section{SUMMER SCHOOLS}

- NanoSteps, Self-organized nano-structures on crystal surfaces, Institut d'études Scientifiques de Cargèse France, June 2008.

- Interfaces between physics and computer science, Jacobs University Bremen, Germany, June 2007.

- Complex Systems and Nonextensive Statistical Mechanics, the Abdus Salam Int. Center for Theo. Physics, Trieste, Italy, August 2006. 
- Cosmology School, Institute for Research in Fundamental Science, Tehran, Iran, August 2004.

\section{TEACHING ASSISTANT (10 semesters)}

- Georg-August-Universitäät Göttingen, Mathematical Methods in Physics, Physics I and II, Quantum Mechanics, Statistical Physics and Thermodynamics.

- Sharif University of Technology,

General Physics I, II and III, Analytical Mechanics, Statistical Physics and Thermodynamics. 